Argonne

\title{
Coupled Calculations in Helical Steam Generators: Validation on Legacy Data
}

Nuclear Engineering Division 


\section{About Argonne National Laboratory}

Argonne is a U.S. Department of Energy laboratory managed by UChicago Argonne, LLC

under contract DE-AC02-06CH11357. The Laboratory's main facility is outside Chicago, at

9700 South Cass Avenue, Argonne, Illinois 60439. For information about Argonne

and its pioneering science and technology programs, see www.anl.gov.

\section{DOCUMENT AVAILABILITY}

Online Access: U.S. Department of Energy (DOE) reports produced after 1991 and a growing number of pre-1991 documents are available free via DOE's SciTech Connect (http://www.osti.gov/scitech/)

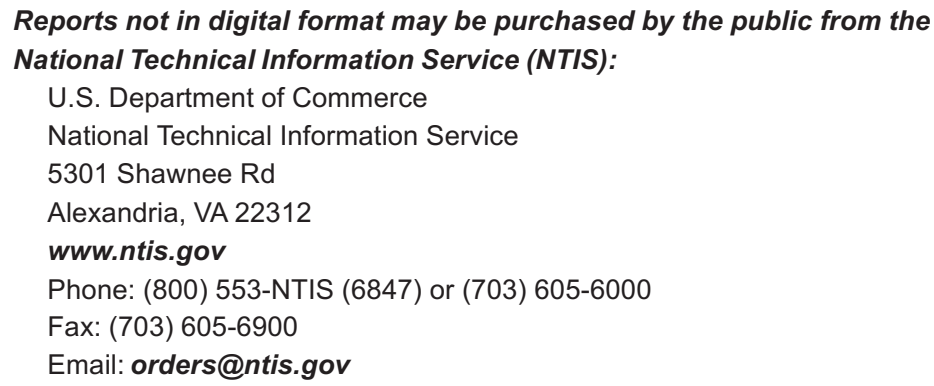

Reports not in digital format are available to DOE and DOE contractors from the Office of Scientific and Technical Information (OSTI):

U.S. Department of Energy

Office of Scientific and Technical Information

P.O. Box 62

Oak Ridge, TN 37831-0062

www.osti.gov

Phone: (865) 576-8401

Fax: (865) 576-5728

Email: reports@osti.gov

\section{Disclaimer}

This report was prepared as an account of work sponsored by an agency of the United States Government. Neither the United States Government nor any agency thereof, nor UChicago Argonne, LLC, nor any of their employees or officers, makes any warranty, express or implied, or assumes any legal liability or responsibility for the accuracy, completeness, or usefulness of any information, apparatus, product, or process disclosed, or represents that its use would not infringe privately owned rights. Reference herein to any specific commercial product, process, or service by trade name, trademark, manufacturer, or otherwise, does not necessarily constitute or imply its endorsement, recommendation, or favoring by the United States Government or any agency thereof. The views and opinions of document authors expressed herein do not necessarily state or reflect those of the United States Government or any agency thereof, Argonne National Laboratory, or UChicago Argonne, LLC. 
ANL/NE-16/49

\section{Coupled Calculations in Helical Steam Generator: Validation on Legacy Data}

E. Merzari, H. Yuan, A. Kraus

Nuclear Engineering Division, Argonne National Laboratory

J. Solberg, R. Ferencz

Methods Development Group, Lawrence Livermore National Laboratory

September 30, 2016 
Coupled Calculations in Helical Steam Generators: Validation on Legacy Data

September $30^{\text {th }}, 2016$ 


\section{SUMMARY}

The NEAMS program aims to develop an integrated multi-physics simulation capability "pellet-to-plant" for the design and analysis of future generations of nuclear power plants. In particular, the Reactor Product Line code suite's multi-resolution hierarchy is being designed to ultimately span the full range of length and time scales present in relevant reactor design and safety analyses, as well as scale from desktop to petaflop computing platforms.

Flow-induced vibration (FIV) is widespread problem in energy systems because they rely on fluid movement for energy conversion. Vibrating structures may be damaged as fatigue or wear occurs. Given the importance of reliable components in the nuclear industry, flow-induced vibration has long been a major concern in safety and operation of nuclear reactors. In particular, nuclear fuel rods and steam generators have been known to suffer from flow-induced vibration and related failures.

Advanced reactors, such as integral Pressurized Water Reactors (PWRs) considered for Small Modular Reactors (SMR), often rely on innovative component designs to meet cost and safety targets. One component that is the subject of advanced designs is the steam generator, some designs of which forego the usual shell-and-tube architecture in order to fit within the primary vessel. In addition to being more cost- and space-efficient, such steam generators need to be more reliable, since failure of the primary vessel represents a potential loss of coolant and a safety concern. A significant amount of data exists on flow-induced vibration in shell-and-tube heat exchangers, and heuristic methods are available to predict their occurrence based on a set of given assumptions. In contrast, advanced designs have far less data available. Advanced modeling and simulation based on coupled structural and fluid simulations have the potential to predict flow-induced vibration in a variety of designs, reducing the need for expensive experimental programs, especially at the design stage.

Over the past five years, the Reactor Product Line has developed the integrated multi-physics code suite SHARP. The goal of developing such a tool is to perform multi-physics neutronics, thermal/fluid, and structural mechanics modeling of the components inside the full reactor core or portions of it with a userspecified fidelity. In particular SHARP contains high-fidelity single-physics codes Diablo for structural mechanics and Nek5000 for fluid mechanics calculations. Both codes are state-of-the-art, highly scalable tools that have been extensively validated. These tools form a strong basis on which to build a flowinduced vibration modeling capability.

In this report we discuss one-way coupled calculations performed with Nek5000 and Diablo aimed at simulating available FIV experiments in helical steam generators in the turbulent buffeting regime. In this regime one-way coupling is judged sufficient because the pressure loads do not cause substantial displacements. It is also the most common source of vibration in helical steam generators at the low flows expected in integral PWRs. The legacy data is obtained from two datasets developed at Argonne and $\mathrm{B} \& \mathrm{~W}$. 


\section{CONTENTS}

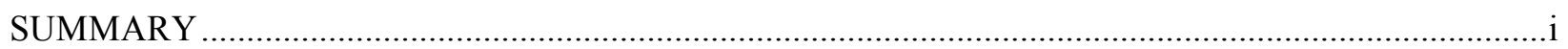

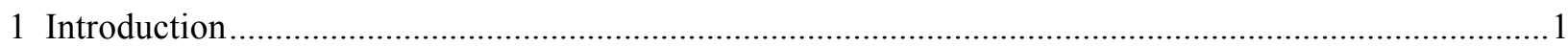

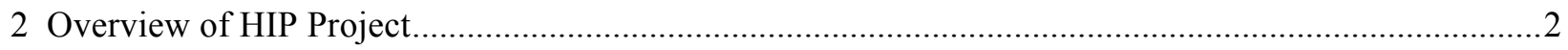

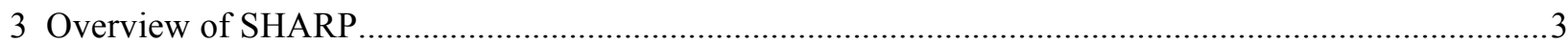

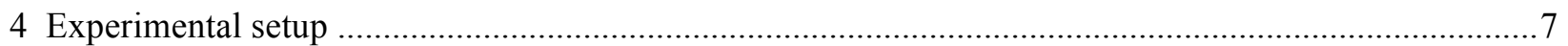

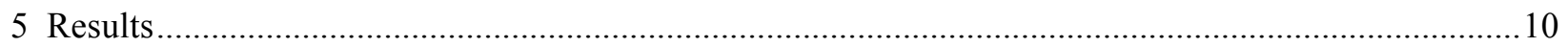

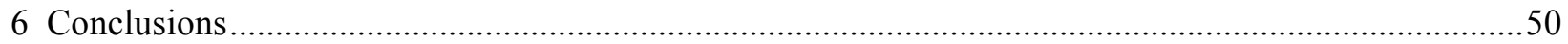

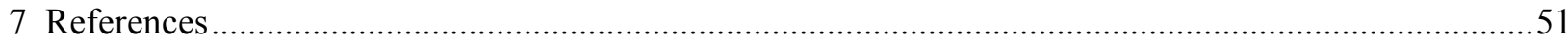




\section{Introduction}

Steam generators (SGs) are an essential component of all pressurized water reactor (PWR) designs. The cost of inadequate prediction of flow-induced vibration (FIV) phenomena within SGs can be crippling: SG units are expensive, and their replacement may cause extended downtimes. Moreover, the loss of public trust produced by radioactive leaks can lead to prolonged shutdowns and even plant closure. The emphasis on reliable SGs has increased in recent years with the growing interest in small modular reactors (SMRs). In fact, with the SG placed within the reactor vessel, the need for a reliable design is increased because the cost of tube failure becomes potentially even more significant.

The focus of this project is the development of a high-fidelity, finite-element analysis/computational fluid dynamics (FEA/CFD) approach to the simulation of FIV based on SHARP [1,2]. Flow-induced vibration is an important limiting factor in the operation of heat exchangers and SGs. Such vibration may cause tube failure because of collisions between oscillating tubes, attrition against support structures (wear), tightness faults against stiff joints, fastener loosening, or fatigue caused by cyclic loads. Hence, the study of FIV is of interest to nearly all vendors and for nearly all reactor designs. While empirical design methods and experience related to FIV might be adequately developed for typical tube bank geometries, design methods and experience related to FIV are far less developed for advanced SG designs, such as those currently considered for SMRs. As a result, numerical simulation or analytical prediction of FIV is even more important for SMRs. For instance, in helical heat exchangers (Fig. 1), vibrations are caused by various coexisting phenomena whose relative importance depends on the flow parameters: turbulent pressure fluctuations, vortex shedding induced by cross-flows, fluid-elastic instabilities, and potentially acoustic vibrations.

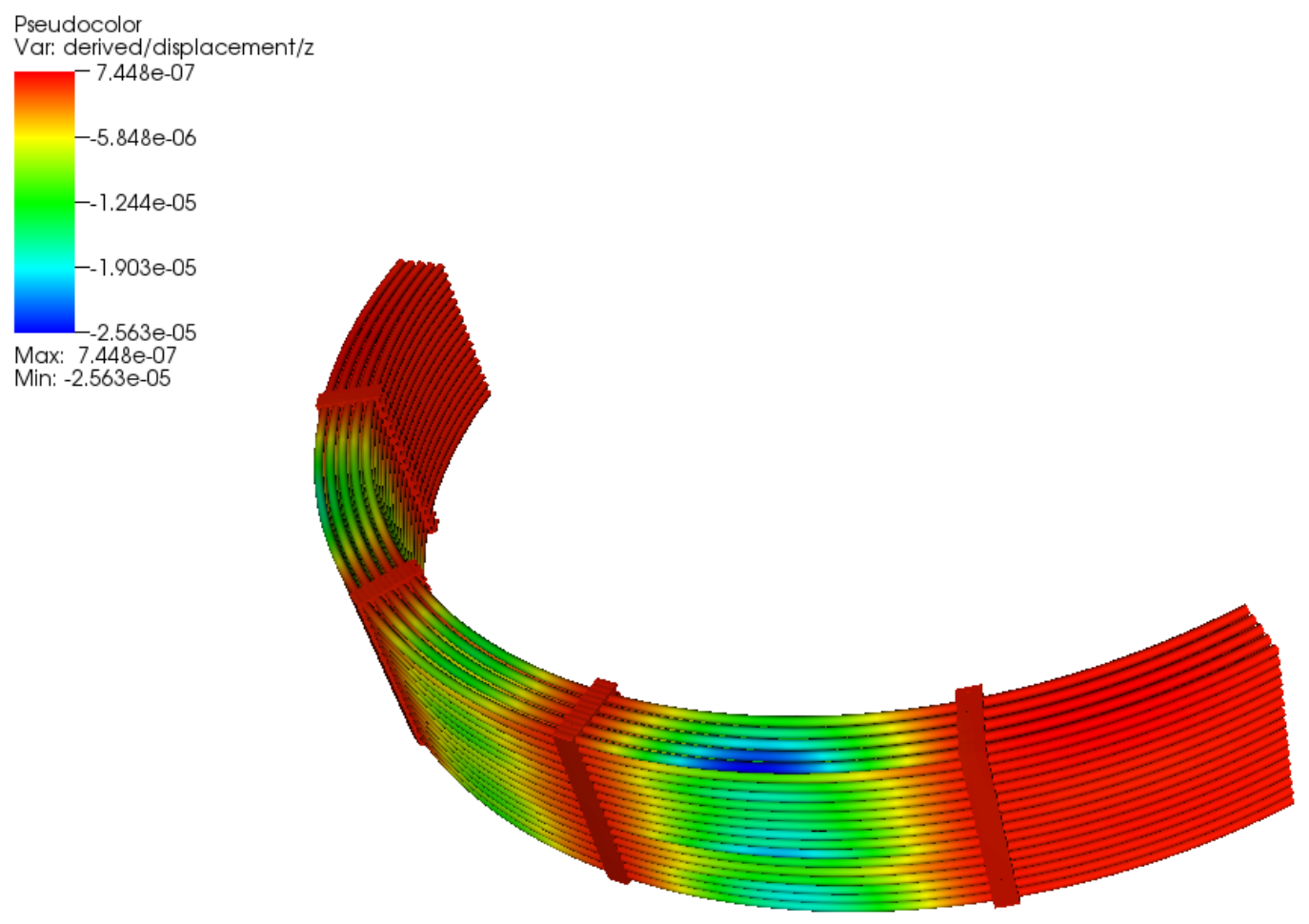

Fig. 1.1 Coupled Nek5000-Diablo simulation of the flow in a helical steam generator. Displacements in $[\mathrm{m}]$. 
An advanced numerical simulation capability for modeling such phenomena will help improve the analysis and evaluation of different design variants in terms of vibrations and heat transfer performance, thus complementing expensive experimental tests and reducing their cost, while developing a better understanding of the physics behind FIV. Work performed in the previous year lead to the development of such a tool in the multi-physics suite SHARP [21], partially validated on classic fluid-structure interactions (FSI) benchmark data $[3,4,5]$.

In this report we focus on the application of SHARP to legacy datasets, in preparation for its application to the NuScale design. We emphasize that these datasets refer to facilities that have been dismantled for over 30 years and the uncertainty for any high-fidelity model will be high. In fact, details such as the structure of the tube supports are practically unknown. Nonetheless the report represents an important stepping stone toward the ultimate goal of simulating helical steam generators. The creation of a highfidelity FSI solver is necessary because the semi-empirical approaches that have been applied to traditional heat exchangers are not applicable to helical coil SGs. Moreover, this high-fidelity approach has the potential for being much more accurate than lower-fidelity FEA/CFD technologies in dealing with FIV, given the complexity of the turbulent flows under investigation. We limit our investigation first to one-way coupling, which is considerably cheaper than tight coupling for this problem and should be used whenever possible.

Simulations are performed here in the turbulent buffeting regime for two datasets: the B\&W dataset [6] and the Chen et al. dataset [7] ("Argonne" dataset in the following). For both cases reasonable damping choices are able to bound the experiment with the calculation results. Moreover the spectral response is in reasonable agreement with the experiment. For the Argonne dataset, a higher flow condition is also simulated, with, as expected, less success. For higher flow rates approaching fluid elastic instability, it is expected that a tightly capability is needed. Overall, the simulations performed here show the complexity of the task at hand and provide important lessons learned that will be valuable in the simulation of the NuScale steam generator design.

\section{Overview of High-Impact Project}

The high-impact project consists of seven tasks categorized under three main areas: code development, verification and validation, and demonstration. The verification and validation part is the largest part of the work and includes experimental activities at Texas A\&M University (TAME), as well as code-to-code comparisons. Table 2.1 summarizes the tasks of the project.

The main objective of Task $\mathbf{A}$ is the implementation of an appropriate interface and coupling capability in SHARP to deal with fluid-structure interaction. Simulations for coupling a stiff structure with a weakly compressible fluid are particularly demanding for the numerical algorithms for both accuracy and robustness. The need for sub-iterations across time steps has been established, and the development of a tight solver has been conducted. More details are provided in Section 3.

The verification and validation of the FSI coupling methodology are part of Task B, which is the primary concern of the present report. This will include only available datasets. This task involves four subtasks:

a) Verification of the coupled methodology (solution transfer). This is part of the focus of this report.

b) Verification/validation against fundamental FSI datasets/cases. It includes in particular verification/validation against fundamental cases (e.g., [3, 4, 5]) normally employed when developing fluid-structure interaction solvers in the literature. 
c) Validation on datasets specific to helical steam generators. This includes datasets for shelland-tube heat exchangers as well as helical SGs [6, 7].

d) Validation on other datasets relevant to nuclear applications [8]. This will be decided in collaboration with AREVA.

The cases discussed in this report fall under categories c). Part c) involves both a validation of the fully coupled methodology and one-way coupled methodology. This report completes the one-way portion of the report.

While work continues on the tightly coupled FIV solver, in order to have impact as soon as possible on the design of helical steam generators for LWR vendors, a one-way coupling approach is essentially delivered here. In this approach the CFD code is used to produce detailed power density spectra for pressure and stresses and to use the spectra as input in the structural mechanics code.

Table 2.1. Task table for the High Impact Problem project.

\begin{tabular}{|l|l|l|}
\hline Task & \multicolumn{1}{|c|}{ Area } & \multicolumn{1}{c|}{ Title } \\
\hline A & Development & $\begin{array}{l}\text { Implementation of an appropriate methodology in SHARP for fluid- } \\
\text { structure interaction simulations. }\end{array}$ \\
\hline B & $\begin{array}{l}\text { Verification \& } \\
\text { Validation }\end{array}$ & $\begin{array}{l}\text { Verification of the methodology implemented in SHARP for fluid- } \\
\text { structure interaction simulations. }\end{array}$ \\
\hline C & Development & Evaluation of different models for the simulation of the secondary. \\
\hline D & $\begin{array}{l}\text { Verification \& } \\
\text { Validation }\end{array}$ & $\begin{array}{l}\text { Modeling and simulation of NuScale experiments related to flow- } \\
\text { induced vibrations. }\end{array}$ \\
\hline E & Demonstration & $\begin{array}{l}\text { Demonstration of SHARP for flow-induced vibration in a helical steam } \\
\text { generator and evaluation of different structural supports. }\end{array}$ \\
\hline F & $\begin{array}{l}\text { Verification \& } \\
\text { Validation }\end{array}$ & $\begin{array}{l}\text { Simulation of flow-induced vibration using STAR-CCM+ (+ FEA code) } \\
\text { for the NuScale steam generator design. }\end{array}$ \\
\hline G & $\begin{array}{l}\text { Verification \& } \\
\text { Validation }\end{array}$ & $\begin{array}{l}\text { PIV and pressure experiments (at TAMU) Numerical simulation for } \\
\text { the validation of the fluid mechanics models. }\end{array}$ \\
\hline
\end{tabular}

\section{Overview of SHARP}

The NEAMS Reactor Product Line (RPL) aims to develop an integrated multi-physics simulation with a multi-resolution hierarchy that ultimately span the full range of length and time scales in relevant reactor design and safety analyses, as well as scales from desktop to petaflop computing platforms. This section discusses the design and the numerical methodologies used in the SHARP toolkit to integrate neutronics, thermal-hydraulics, and structural mechanics physics components to perform coupled reactor analysis on a representative SMR/SFR?? core geometry. Based on the requirements specified, a problem to quantify the primary structural mechanical feedback effect with multi-way coupling has been implemented with dual resolution: a detailed heterogeneous model represents the duct surrounding each assembly while the interior of the ducts (the individual assemblies) is represented with a homogenized geometry.

In order to produce a fully coupled-physics simulation capability, two obvious approaches can be pursued. In one approach, existing single-physics codes/components can be assembled into an overall 
coupled simulation code with appropriate interfaces to communicate between the components to capture the nonlinear feedback effects. This is generally referred to as a "small-f" or "bottom-up" framework approach $[9,10]$. The other approach is to use an integrated, coupled-physics modeling framework, with new code pieces for each relevant physics area developed inside that framework from scratch. This is sometimes referred to as a "large-F" or "top-down" approach [11]. The primary advantage of the former approach is that it preserves several man-years invested in existing verified and validated individual physics modeling codes, but at the cost of some intrusive modifications to enable the software interfaces. The large-F approach avoids intrusive interfacing by providing a unified platform to enable coupling, but at the cost of rewriting all the necessary physics codes and verifying the components individually and as a whole. The overall approach being pursued in the RPL effort is to develop and demonstrate a small-f framework for performing coupled multi-physics analysis of reactor core systems. This system takes advantage of many single-physics codes also sponsored by the overall NEAMS program over the past several years.

Details regarding the background of construction of the RPL coupled-physics framework (SHARP), and the methodology are discussed in the following sections.

\subsection{The SHARP Multi-physics Code System}

A multi-physics reactor core modeling code can be constructed in many ways, and numerous efforts have provided stepping-stones for future efforts [10]. What distinguishes the SHARP effort from others is the goal of flexibility in the physics, discretization types, and software options supported by the framework. This section describes the SHARP modeling approach in detail and illustrates how various physics codes have been connected to this framework.

SHARP employs a "bottom-up" approach, so it can use existing physics codes and take advantage of existing infrastructure capabilities in the MOAB framework and the coupling driver/solver library, the Coupled Physics Environment (CouPE), which utilizes the widely used, scalable PETSc library.

Using an existing physics code in this system (Fig. 3.1) requires that the system support the mesh type used by the individual physics models. The physics models can retain their own native representation of the mesh, which gets transferred to and from MOAB's representation through a mesh adaptor; or it can use MOAB's representation directly. Language interoperability through the C/Fortran-based iMesh interfaces also allows flexibility in the implementations that are tuned to individual physics requirements without overhead.

In practice, this means that the coupled system may be solved on multiple meshes, each of which models part or all of the physical domain of the problem. In order to perform efficient coupled calculations, the results must be transferred from the mesh on which they are generated (source mesh), to the mesh for which they provide initial or boundary conditions (target mesh) due to nonlinearity introduced because of coupling between physics models. "Multi-way" transfer is required in cases where the physics depend on each other's solution fields, for example in reactor analysis where neutronics computes heat generation based on temperature properties computed by thermal-hydraulics, which in turn depends on the heatgeneration source term computed by neutronics. 


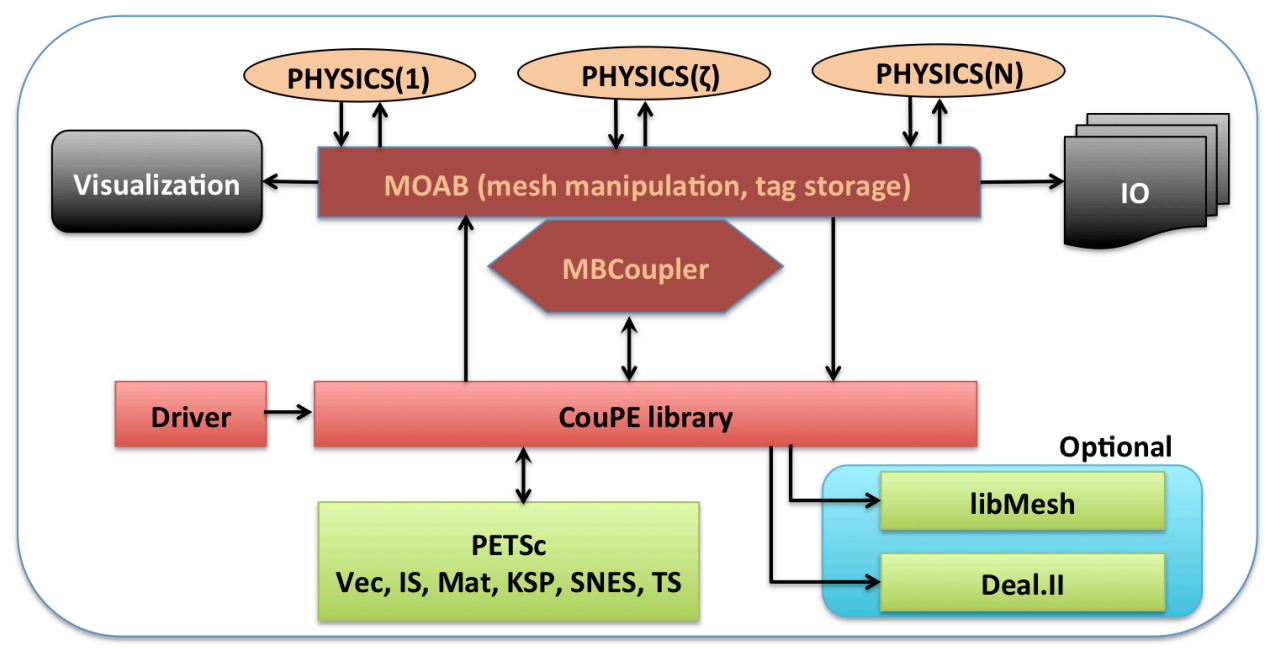

Figure 3.1 SHARP architecture

Since relevant physics components solving a nuclear engineering problem have widely varying backgrounds in terms of code architectures, dependency requirements, and specialized solver datastructures, a flexible approach to the coupling methodology was necessary in order to obtain accurate solutions. This motivation led to the development of the MOAB-based spatial projection tools and the CouPE drivers based on PETSc library to orchestrate the global nonlinear solver. Details regarding these tools are given in the following sections.

\subsection{SHARP Physics Components}

In the SHARP framework, MOAB interfaces are implemented for these physics components that are relevant to fast reactor physics analysis. The addition of a new physics component to the framework requires integration and ability to read the mesh and possibly associated data from iMesh/MOAB formats, along with implementation to propagate solution variables back onto the mesh after their computation via tags defined either on discrete vertices or on elements. Because of the various storage formats used in physics models and the parallel domain-decomposed environment in which these calculations are usually run, this integration process can be involved.

In order to better understand the level of fidelity that can be achieved by the SHARP framework, some key aspects of these physics components are given below.

\subsubsection{Computational Fluid and Thermal Dynamics Solver (Nek5000)}

The Nek5000 $[12,13]$ computational fluid dynamics solvers are based on the spectral-element method developed by Patera [14]. Nek5000 supports two formulations for spatial and temporal discretization of the Navier-Stokes equations. The first is the $\mathrm{P}_{N-}-\mathrm{P}_{N-2}$ method with velocity/pressure spaces based on tensor-product polynomials of degree $N$ and $N-2$, respectively. The second is the low-Mach number formulation of Tomboulides et al. $[15,16]$, which uses consistent order-N approximation spaces for both the velocity and pressure. The low-Mach number formulation is also valid at the zero-Mach (incompressible) limit. The Nek5000 code has been extensively verified and validated for several benchmark problems and has a proven scalability on existing petascale architectures up to 131,072 processors (over a billion degrees-of-freedom). 
Of particular relevance to fluid-structure interaction is the Arbitrary Lagrangian Eulerian (ALE) modeling capability in Nek5000. For time-dependent geometry problems, a mesh velocity is defined at each collocation point of the computational domain (mesh) to characterize the deformation of the mesh. In the solution of the mesh velocity, the value of the mesh velocity at the moving boundaries is first computed by using appropriate kinematic conditions (for free-surfaces, moving walls and fluid layers) or dynamic conditions (for melting fronts). On all other external boundaries, the normal mesh velocity on the boundary is always set to zero. In the tangential direction, either a zero tangential velocity condition or a zero tangential traction condition is imposed; this selection is automatically performed by Nek5000 based on the fluid and/or thermal boundary conditions specified on the boundary. Under special circumstances, however the user may want to override the defaults set by Nek5000. If the zero tangential mesh velocity is imposed, then the mesh is fixed in space; if the zero traction condition is imposed, then the mesh can slide along the tangential directions on the boundary. The resulting boundary-value-problem for the mesh velocity is solved in Nek5000 by using an elastostatic solver, with the Poisson ratio typically set to zero. The new mesh geometry is then computed by integrating the mesh velocity explicitly in time and updating the nodal coordinates of the collocation points. Note that the number of macro-elements, the order of the macro-elements and the topology of the mesh remain unchanged even though the geometry is time-dependent. The use of an ALE description in Nek5000 ensures that the moving fronts are tracked with the minimum amount of mesh distortion; in addition, the elastostatic mesh solver can handle moderately large mesh distortion. However, the user is responsible deciding when a mesh would become "too deformed" and thus requires remeshing. The execution of the program will terminate when the mesh becomes unacceptable, that is, when a one-to-one mapping between the physical coordinates and the isoparametric local coordinates for any macro-element no longer exists. In general this is considered sufficient for flow-induced vibrations because up to the onset of fluid-elastic instability deformations will be modest. In any case the mesh for the cases presented in this report is fixed, and the ALE solver is not invoked.

\subsubsection{Solid Mechanics Solver (Diablo)}

The Diablo code being developed at Lawrence Livermore National Laboratory uses implicit, Lagrangian finite-element methods for simulating solid mechanics and multi-physics events over moderate to long time frames [17]. A primary focus is nonlinear structural mechanics and heat transfer. The code provides a venue for applying parallel computation to discretization technologies developed and user-tested in the legacy serial-processor codes NIKE3D and TOPAZ3D. Diablo is built around Fortran 95 data structure objects and a message-passing programming model. The architecture provides flexibility for the addition of other field problems, such as electromagnetics.

In structural analysis of mechanical assemblies, a key functionality is "contact": capturing the interaction between unbonded material interfaces. The Diablo team has broad experience with contact problems and has created state-of-the-art algorithms for their solution. Their experience with contact motivates the use of low-order spatial discretizations, such as eight-node hexahedra for continua and four-node quadrilaterals for shells. Appropriate formulations are employed to accommodate nearly incompressible material models, such as for metal plasticity and rubber elasticity. Global algorithms include second-order and quasi-steady time integration and a number of approaches for nonlinear iteration: full Newton, modified-Newton, multiple quasi-Newton updates, and line search. Linear solvers are utilized from multiple libraries.

\subsubsection{One-Way Coupling Methodology for Flow-Induced Vibrations}


At times specified by the Nek user, Nek results along the tube surfaces are dumped to file(s) as pressure data for each 9-node quad on the tube surfaces. Using a sequence of Fortran utility programs, these pressure values are mapped to Neumann boundary surfaces within the Diablo mesh, and then the collection of time-dependent data is packed as nodal surface time histories into Exodus binary files. These files are then read in during the solution process and the data sampled and interpolated for the purpose of providing Neumann pressure loads to Diablo. Diablo time steps and Nek time steps need not be commensurate. A 1-1 relationship must exist between each 9-node quadratic facet on the Nek side of the boundary and a collection of four 4-node quads on the Diablo side of the interface. In the future this restriction will be eliminated. A time-averaged pressure file is also produced, mapped, and translated into an Exodus binary file, but containing only a single time history. Before the solution of the dynamic Diablo solution, a quasi-static Diablo run is performed by using the time-averaged pressure profile and is used as the initial condition for the Diablo time-dependent solution. This eliminates any "kick" due to the sudden application of the pressure profile, and it produces acceleration data that reflects the quasi-steadystate behavior. For the specific cases under consideration, the structural dynamics have been treated as linear, hence only a single LHS is formed and factored by the direct linear solver, which improves speed. If nonlinear effects are to be included, full Newton iterations can be performed, or various quasiNewton schemes can be employed to reduce the time spent in linear algebra.

\section{Experimental Setup}

The legacy data is obtained from two datasets: the so-called Argonne dataset and a dataset from the B\&W company.

\subsection{Argonne Dataset}

Chen et al. [7] performed an experiment on a half-scale sector model of a steam generator helical coil tube tank in Argonne National Laboratory in the 1980s. This test was designed to study only the structure motion of the whole tube bundle under flow conditions. Tube vibrations under different flow conditions are recorded.

The test section is a half-scale, 135-degree sector model of the steam generator of a liquid metal fast breeder reactor. The model is designed to analyze the vibration of tubes only, rather than the vibration of the whole helices. The test section is shown in Fig. 4.1 with the outer shell cover removed. The model contains three spans of helical tubes to simulate the exterior of seven columns of the steam generator.

The test section has 30 rows of tubes in the flow direction (downward). Hanger bars support these tubes at an interval of 45-degree, splitting the test section into three 45-degree spans. Flow is directed only across the center span.

Fig. 4.2 shows the radii of curvature of the seven columns of tubes. Hang bars at A, B, C, and D support each tube, leaving two ends open to the atmosphere. The tube patterns at supports A and B are presented in Fig. 4.3. We can see in Fig. 4.3, the tube patterns are not exactly aligned along the tube coil line. Therefore, the tube patterns are considered exact same at different hanger bars in order to simplify geometry construction in our simulation. The tube arrays consist of stainless steel tubes with $0.01588 \mathrm{~m}$ (5/8 inch) outer diameter, and $0.00089 \mathrm{~m}(0.035 \mathrm{inch})$ wall thickness. The tubes in the transitional region (these tubes are denoted by double circles in Fig. 4.3) are heavy wall tubes with wall thickness of 0.0165 $\mathrm{m}(0.065 \mathrm{inch})$ to increase the stiffness. The pitch between adjacent columns is constant at $0.0254 \mathrm{~m}(1$ inch). The pitch within each column is constant at $0.0238 \mathrm{~m} \mathrm{(15/16} \mathrm{inch).}$ 
The motion of the tubes was measured by means of accelerometers installed on spring mounts inserted in the tubes. The accelerometer has two sensitive axes, one in the out-of-plane direction (drag direction) and the other in the in-plane direction (lift direction).

Several tests have been conducted using air and water. However, only the data from the water test will be used to compare with numerical data. The inlet flow velocity distribution is uniform with the screen flow equalizer. With the exception of positions close to the outer wall, the deviation is less than $10 \%$ and is considered to be satisfactory. Despite the slight deviation from uniform flow velocity in experiments, we applied a uniform flow velocity at the inlet of simulation domain.

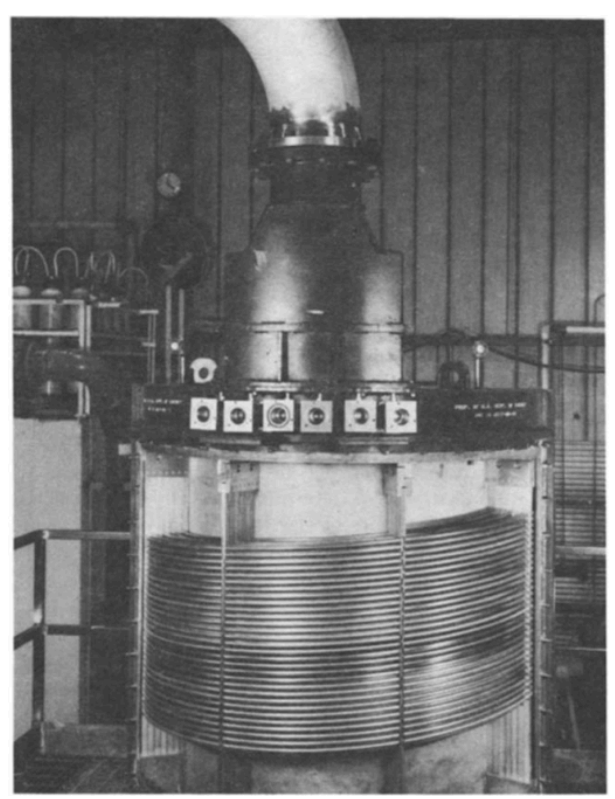

Fig. 4.1. Picture of the experimental setup. From [7].

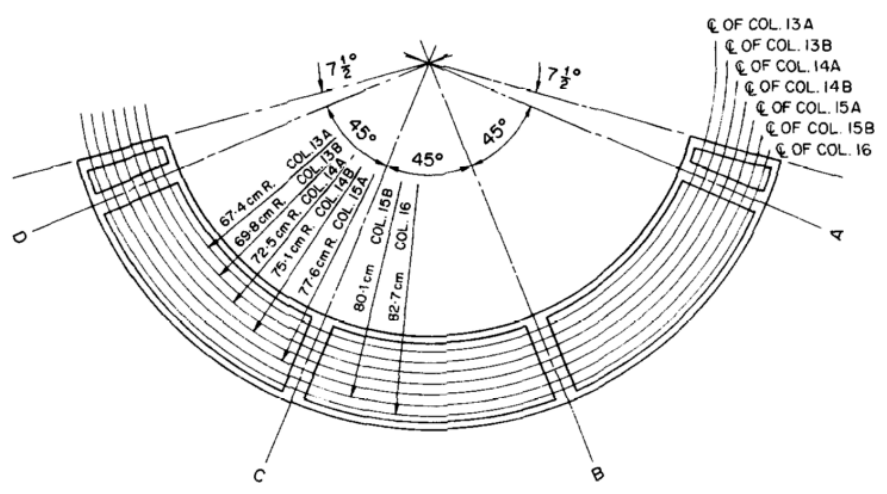

Fig. 4.2 Radii of curvature of helical tubes, describing the position of the supports. From [7]. 


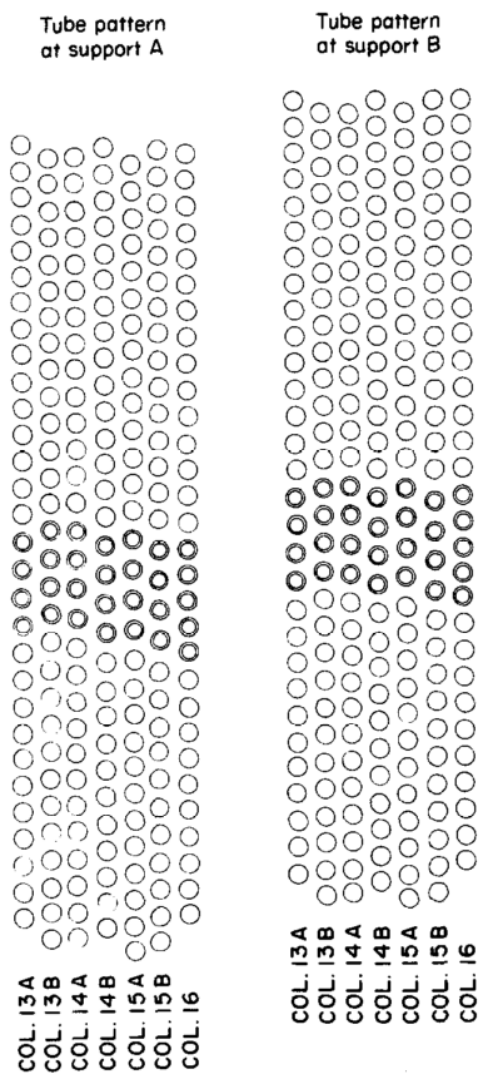

Fig. 4.3 Tube patterns at supports A and B. From [7].

\subsection{B\&W Dataset}

The B\&W experimental facility is described in detail in [6]. The facility was designed to study flowinduced vibration in a 150 degree sector of a helical steam generator. Only one-sixth of the tubes was included (layers 25 through 30). The sector includes 5 sections, three of which are subjected to fluid flow. Between each section a set of supports was present, better described than in the Argonne case (Section 4.1).

The cross section is regular and much simpler than the one shown in Fig. 4.3. The bundle corresponded to a regular lattice 5-1/2 tubes wide and 16 tubes deep. The half tubes were employed to measure the effect of the $25^{\text {th }}$ layer of tubes on the flow distribution of the remaining five. The half-tubes were merged with the inner wall and were not simulated in the structural model. The pitch between both directions was equal to $0.0254 \mathrm{~m}(1 \mathrm{inch})$. Each tube had a diameter of $0.01905 \mathrm{~m}(0.75 \mathrm{inch})$. The outer diameter coil was 2.67335 meters. 


\section{Results}

\subsection{Argonne Dataset}

Three types of simulations were conducted: fluid, structural, and coupled

\subsubsection{Fluid Simulations}

For the Nek5000 fluid simulations, a mesh involving approximatively 3,000,000 elements was constructed. The number of grid points involved went from $\sim 125,000,000$ at low polynomial order to $\sim 1,500,000,000$. Details of a coarse mesh are illustrated in Fig. 5.1. In this mesh, at low poly1 omial order ( $N=4$, corresponding to $1 \mathrm{x} 1=5$ points per element), the maximum $\mathrm{y}+$ at tube surface is around 8.0 , and the minimum $\mathrm{y}+$ is around 0.01 . At high polynomial order $(N=7$, corresponding to $1 \mathrm{x} 1=8$ points per element), the $\mathrm{y}+\mathrm{a}$ the surface is below 1.0. Fig. 5.2 shows the computational domains for the Argonne tests. Since only the center section has flow, we simulate the flow only in the center section. More refined meshes have also ben analyzed.

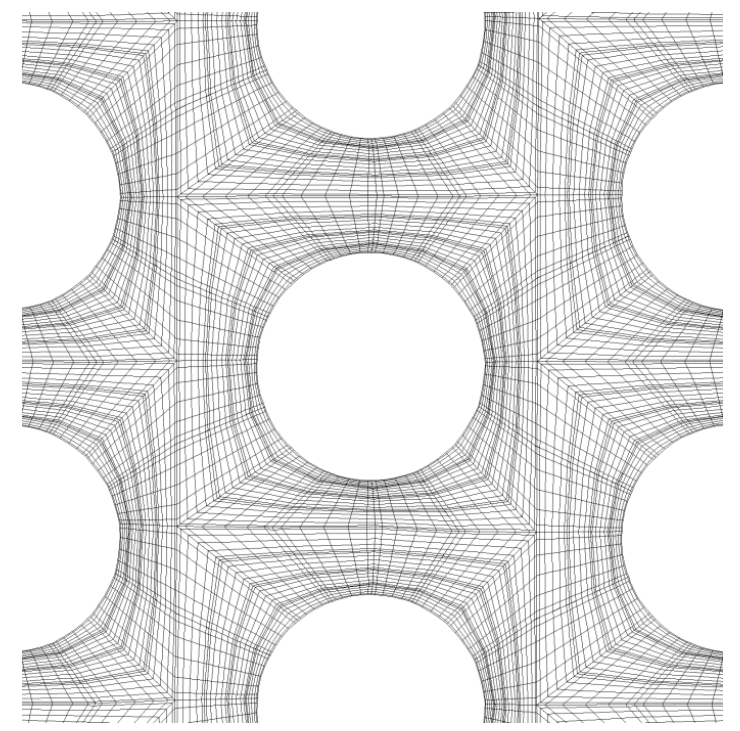

Fig. 5.1. Mesh for Argonne case, low polynomial order. 


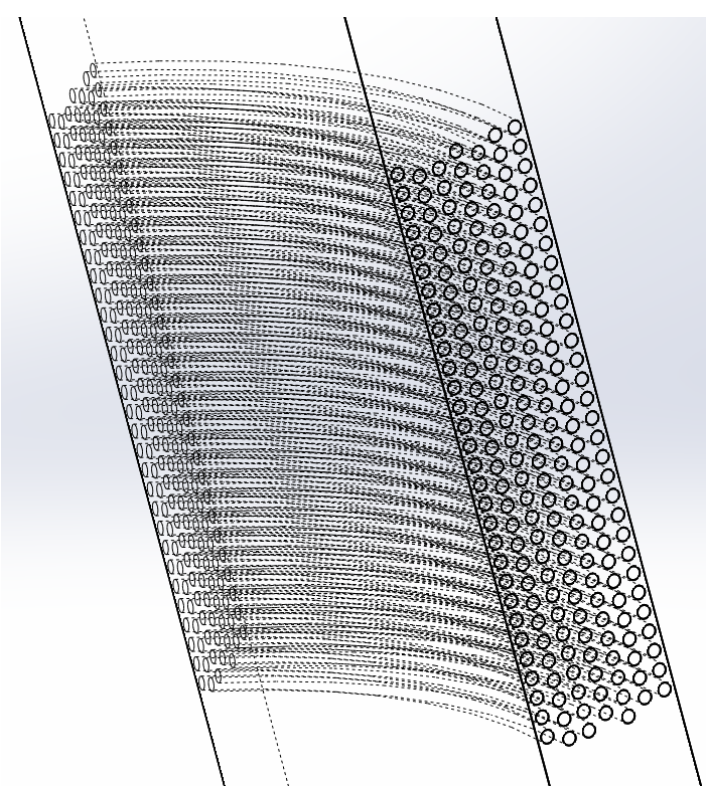

Fig. 5.2. Computational domain for the Argonne case (flow goes downward).

In the experiment of Chen et al. [7], a screen flow equalizer was placed at the inlet of test section. This screen flow equalizer produced a fairly uniform flow velocity distribution. However, it uses multiple screen wires to unify the flow, which also introduce small eddies. The size and frequency of eddies are associated with the diameter of scree wire diameter, which is $0.2 \mathrm{~mm}$ in Chen et al.'s experiment [7]. These eddies introduce a fluctuation of upper plenum pressure. However, the frequency of pressure fluctuation in the upper plenum observed in experiment has a much higher value than tube vibration frequency. In our current simulation, a Synthetic-Eddy-Method (SEM) is used to mimic the behavior of the screen flow equalizer. Fig. 5.3 is a snapshot of the velocity magnitude in the upper plenum. It shows how SEM inlet condition mimics the behavior of the screen flow equalizer. SEM inlet condition creates multiple eddies with a given size, $0.2 \mathrm{~mm}$ in this case. Fig. 5.4 presents the pressure power spectrum in the upper plenum for one of our simulations. As we can see in Fig. 5.4, the dominant frequency of pressure fluctuation is around $1 \mathrm{kHz}$, which is close to the experiment observation (Figure 17 in [7]). 


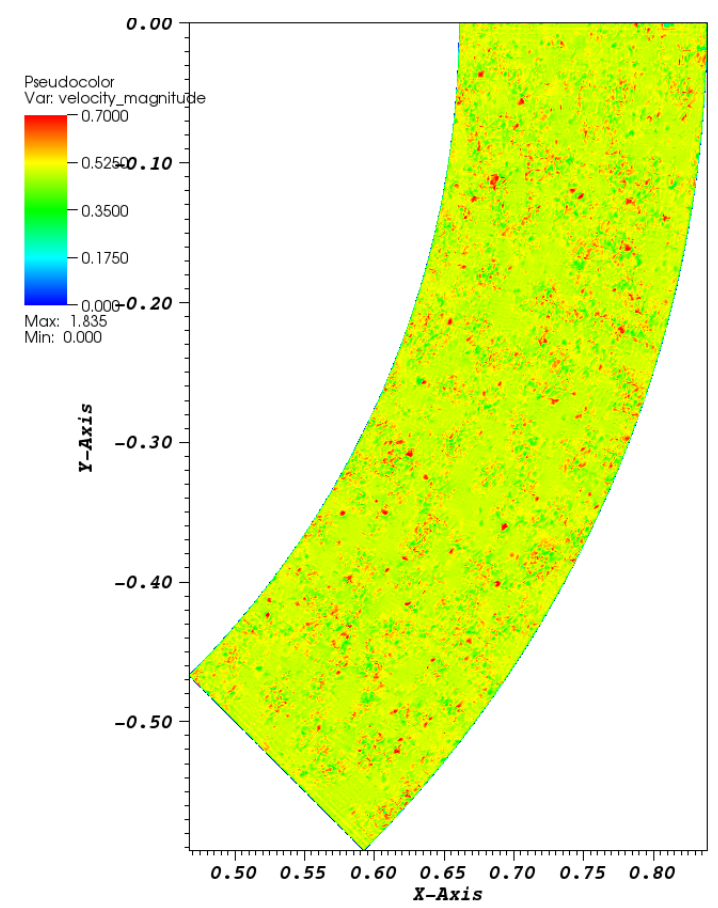

Fig.5.3 Snapshot of velocity in upper plenum

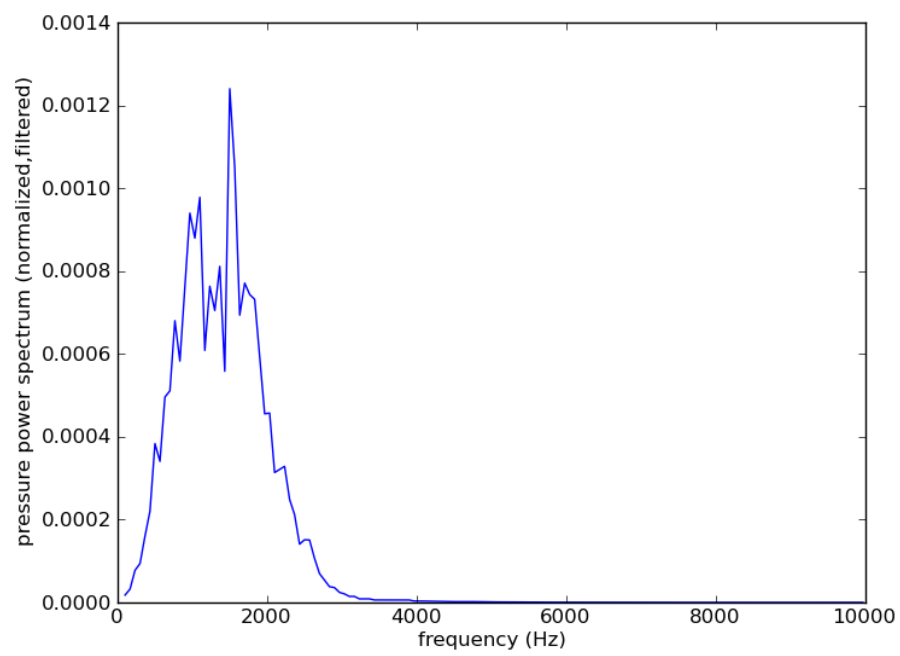

Fig. 5.4 Power spectrum of pressure fluctuation in upper plenum

In the flow field, we have two sampling lines, as indicated in Fig. 5.5. The mean flow paths between tubes are numbered as channels 1 to 6 (from left to right in Fig. 5.5). One sampling line is at the center of channel 1, and the other line is at the center of channel 4. For the data comparison in this section, we tested only the case with inlet average velocity of $0.49 \mathrm{~m} / \mathrm{s}$. This inlet velocity corresponds to the average gap velocity of $1.28 \mathrm{~m} / \mathrm{s}$. The average gap velocity in tube bundles is used to indicate each case in [7], and we follow that in this report. 
The data from Nek large eddy simulation (LES) are compared with that from STAR-CCM+ simulation by using URANS. These simulations were performed using a version of the standard $k-\varepsilon$ model for turbulence that incorporates realizability constraints and a quadratic constitutive relation. This model provides a relatively fast, robust solution approach, and attempts to capture some of the Reynolds-stress anisotropy expected for this geometry. An adaptive "All-y+" approach was used for wall modeling, and the wall cells were all generally between $30<\mathrm{y}+<100$. A case was run with a refined wall mesh $\left(\mathrm{y}^{+} \sim 1\right)$ but this did not significantly affect results.

As the main flow is going to minus $\mathrm{z}$ direction, when we present velocity in the $\mathrm{z}$ direction, we take its negative value. Fig. 5.6 presents the average velocity comparison of Nek and STAR in each direction along the center of channel 1. The total average time for the Nek simulations is around 3 seconds, and for STAR is around 1 second. As we see, the averaging time is likely insufficient to reach completely converged statistics. Fig. 5.7 shows the variance of velocity in each direction along the center of channel 1. As we expected, the URANS under-predicts the velocity fluctuation compared with the LES case. Fig. 5.8 and 5.9 exhibit the same data but for channel 4 rather than channel 1 . We reach the same conclusion from the data in channel 4. In figures 5.5 to 5.9 , data from different polynomial orders $(N=4$ and $N=6$, corresponding to $1 \times 1=5$ and $1 \times 1=7$ points per element)is also compared with each other. The average velocities do not show significant difference between polynomial orders, indicating that the lower polynomial order is likely sufficient. However, the velocity variance exhibits some sensitivity to the polynomial order indicating that higher resolution is likely needed in order to resolve adequately all relevant turbulence scales. 


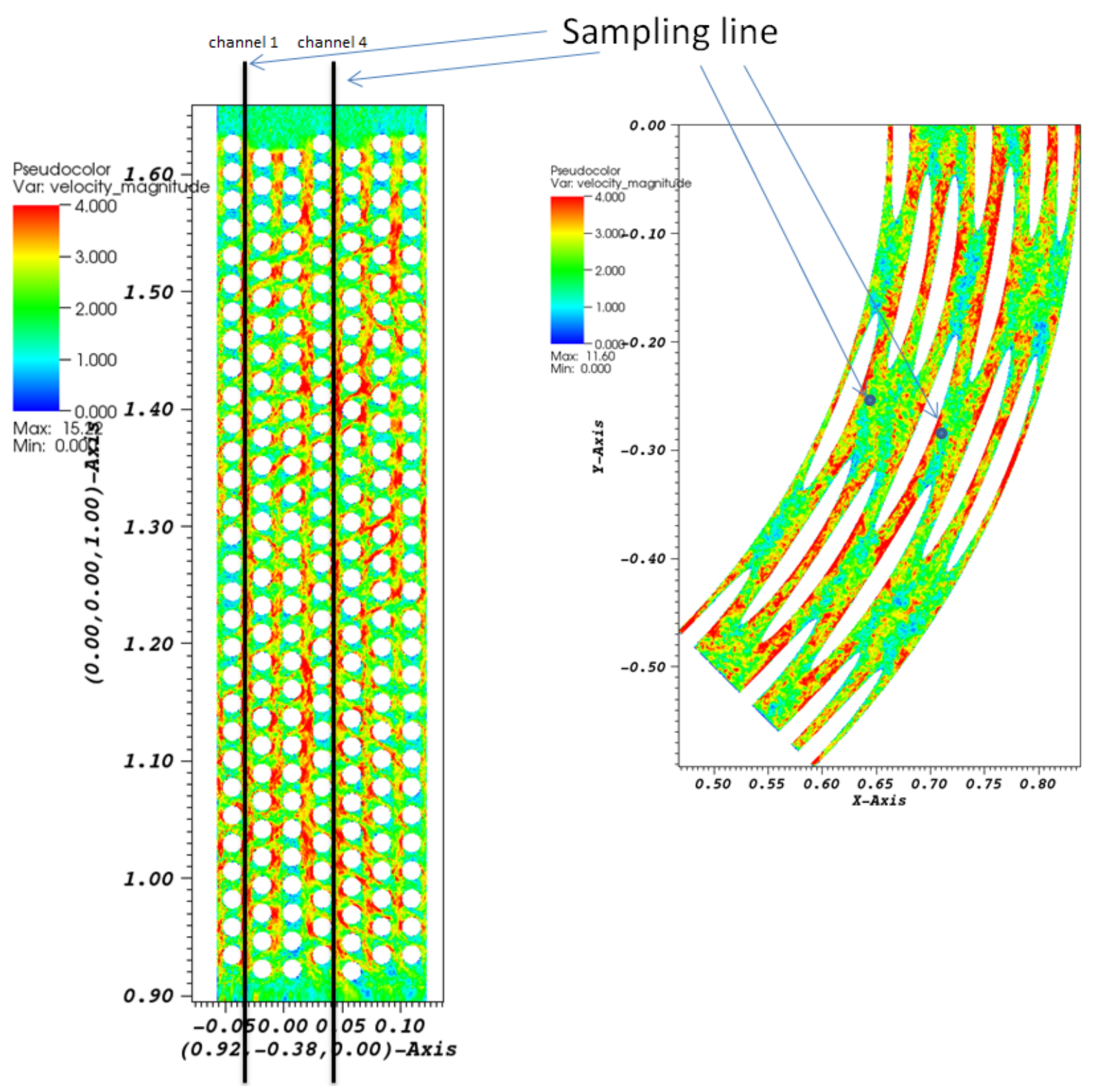

Fig. 5.5. Snapshot of the flow field and sampling line locations. 

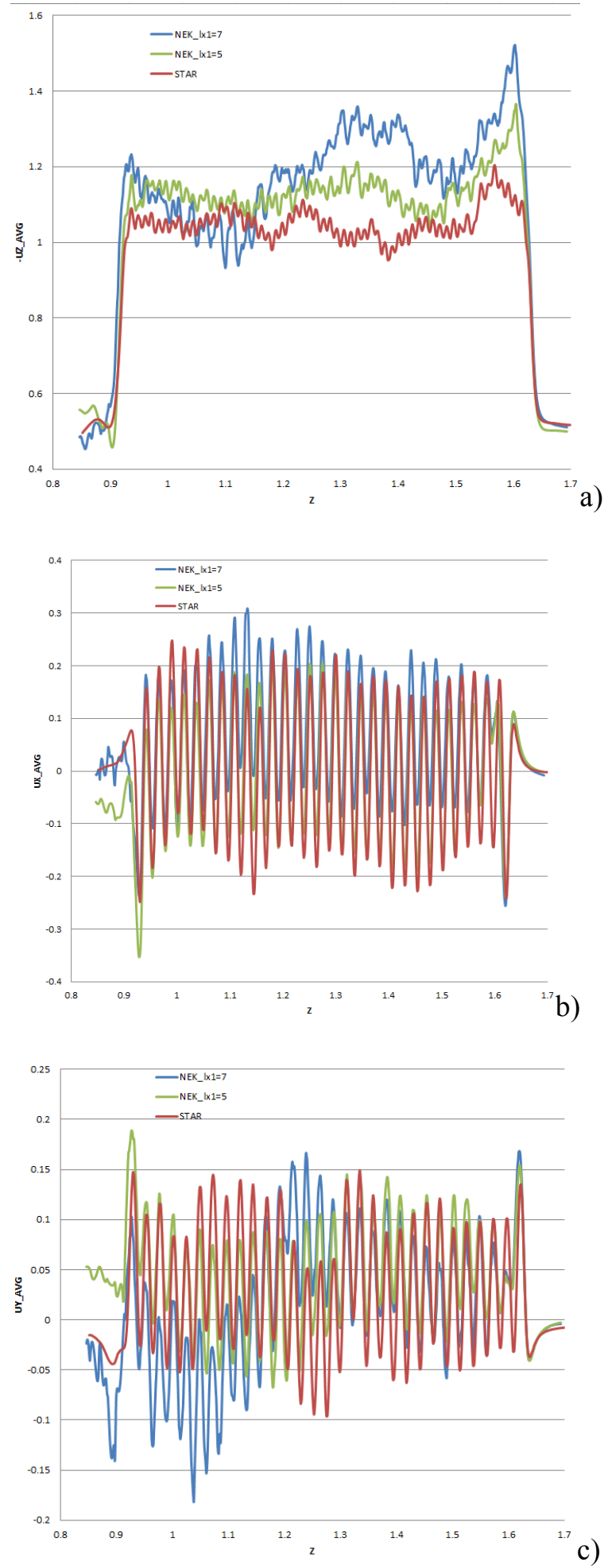

Fig. 5.6 average velocity in channel 1 (flow goes from right to left). a) z-direction (streamwise), b) xdirection, c) y-direction. 

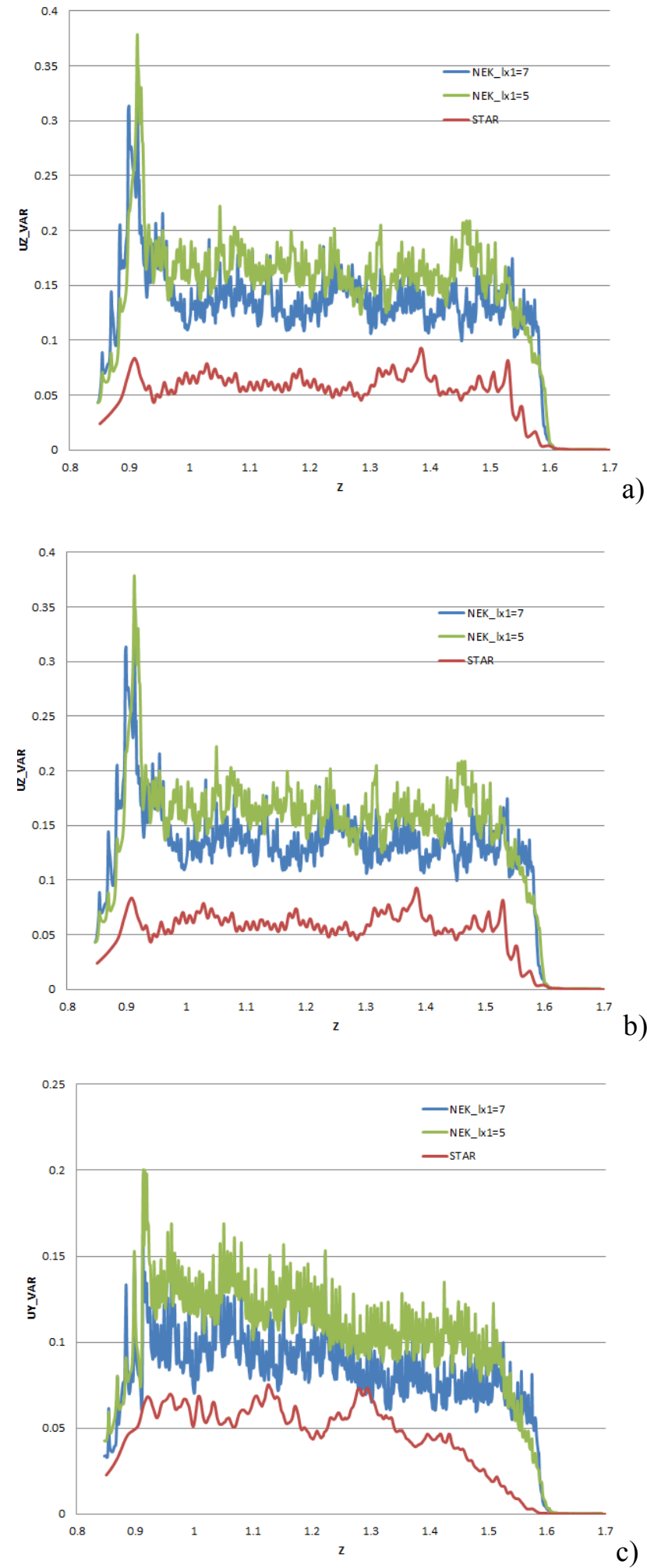

Fig. 5.7. Velocity variance in channel 1 (flow goes from right to left). a) z-direction (streamwise), b) x-direction, c) y-direction. 

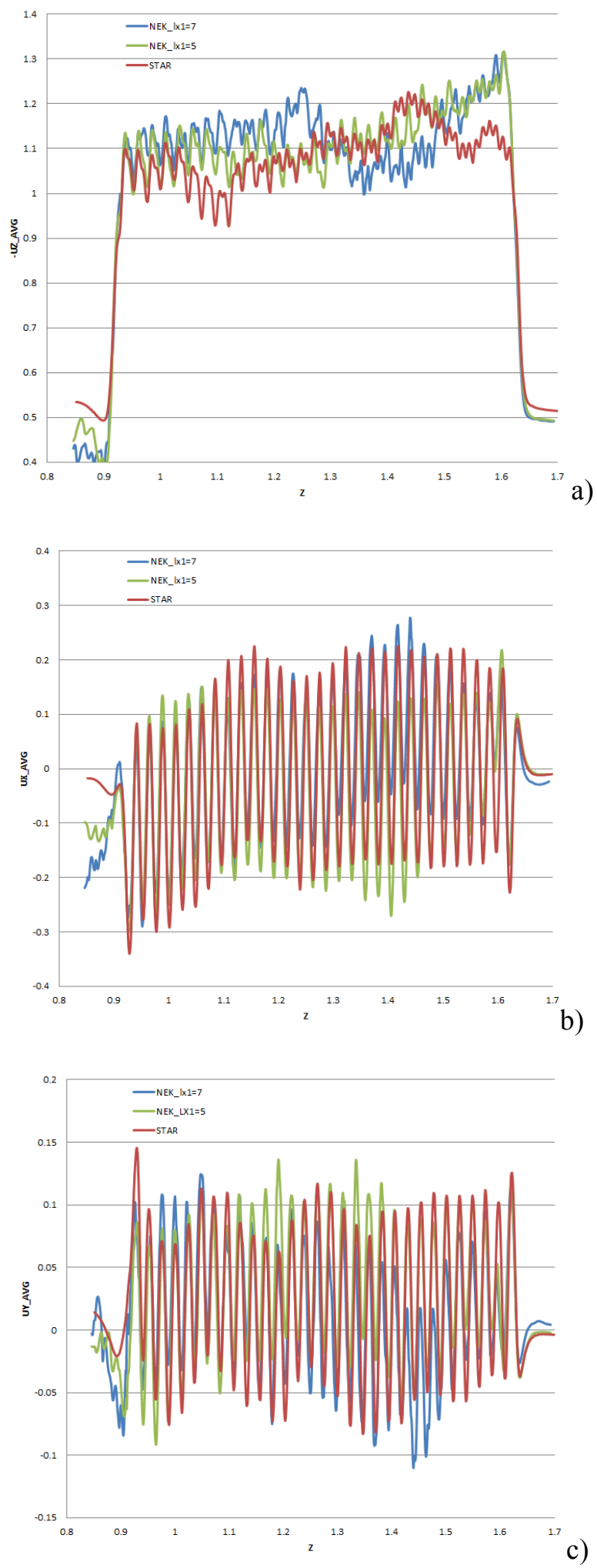

Fig. 5.8. Average velocity in channel 4 (flow goes from right to left). a) z-direction (streamwise), b) x-direction, c) y-direction. 

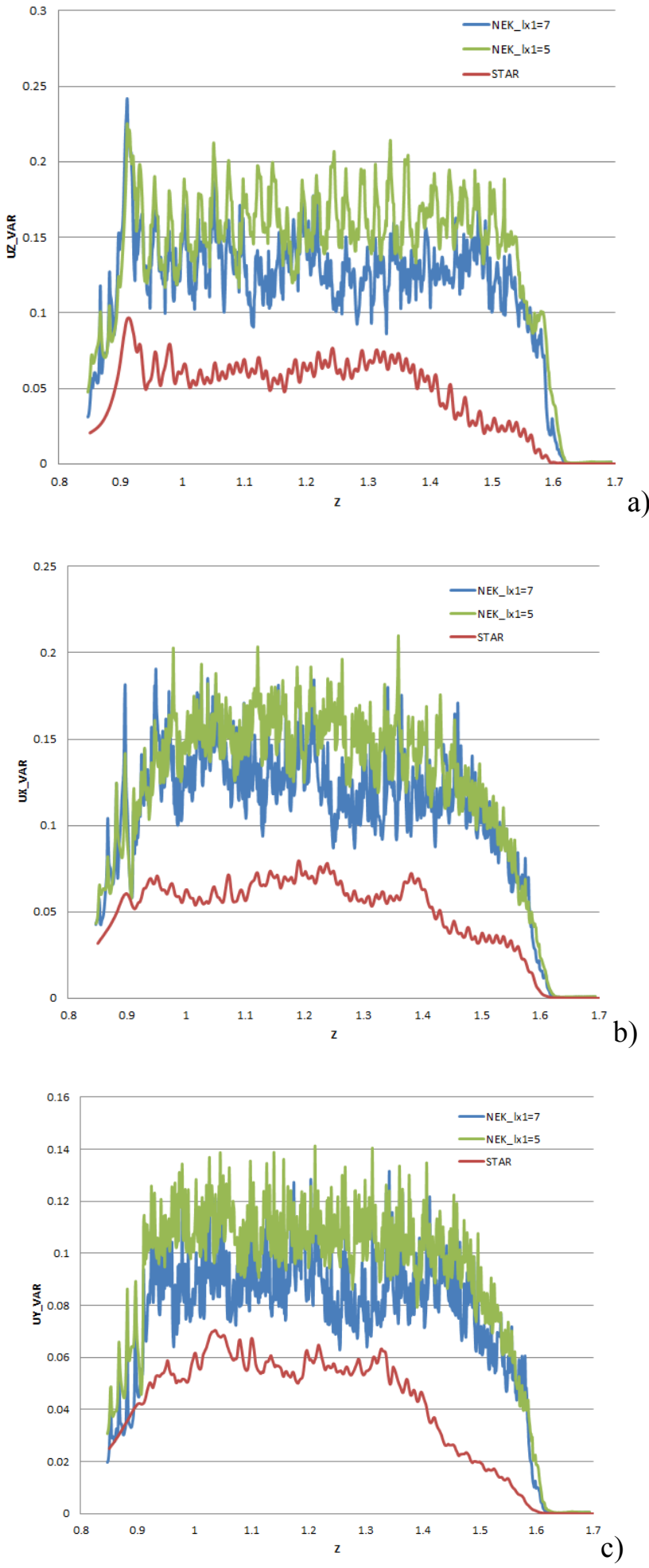

Figure 5.9. Velocity variance in channel 4 (flow goes from right to left). a) z-direction (streamwise), b) $x$-direction, c) y-direction. 
Because the helical coil tubes, the secondary flow inside the steam generator is complicated. As the bulk flow meets the helical tubes, it starts to follow the curvature of tubes. However, because of the wall on the side of the flow section, secondary flow bounds back at the side. As a result, we observe a secondary flow that acts like several big eddies inside the bundle of tube. Because of these eddies, when we take a snapshot of velocity just after the tube bundle, there are two major eddies that flow in different directions.

Figures 5.10 to 5.13 present the average velocity snapshot (converted to cylindrical coordinate) at different axial locations along the streamwise direction. As we can see, as the flow proceeds farther into the tube bundle, two eddies dominant the secondary flow. For example, in figures 5.18, 5.12, 5.13, there is an obvious movement in azimuthal velocity. In the upper part, there is a movement in anti-clockwise direction. At the same time, the movement is in clockwise direction in the lower part. The secondary flow inside the tube bundle results a similar flow pattern as flow exits the tube bundle, as shown in Fig. 5.14.

This secondary flow could enhance heat transfer but bring additional tube vibration and pressure drop. However, this secondary flow has a lower velocity magnitude compared with that of the streamwise flow, hence its impact may be small.

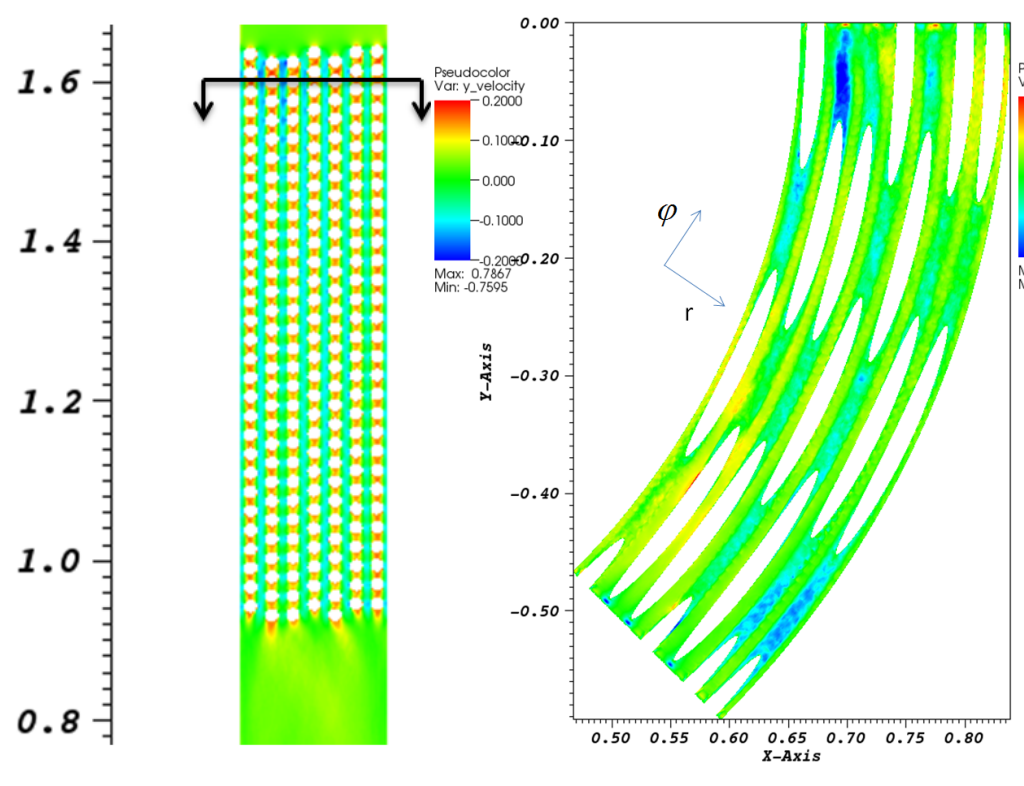

(a) (b)

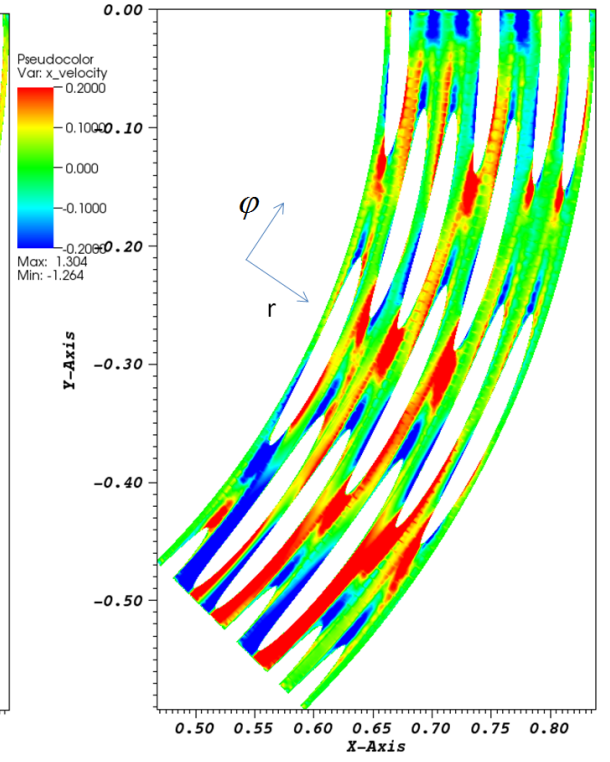

(c)

Fig. 5.10. Average velocity snapshot in axial cross section $(Z=1.6 \mathrm{~m}$, flow goes downward $)$. a) cross section location, b) azimuthal velocity, c) radial velocity. 


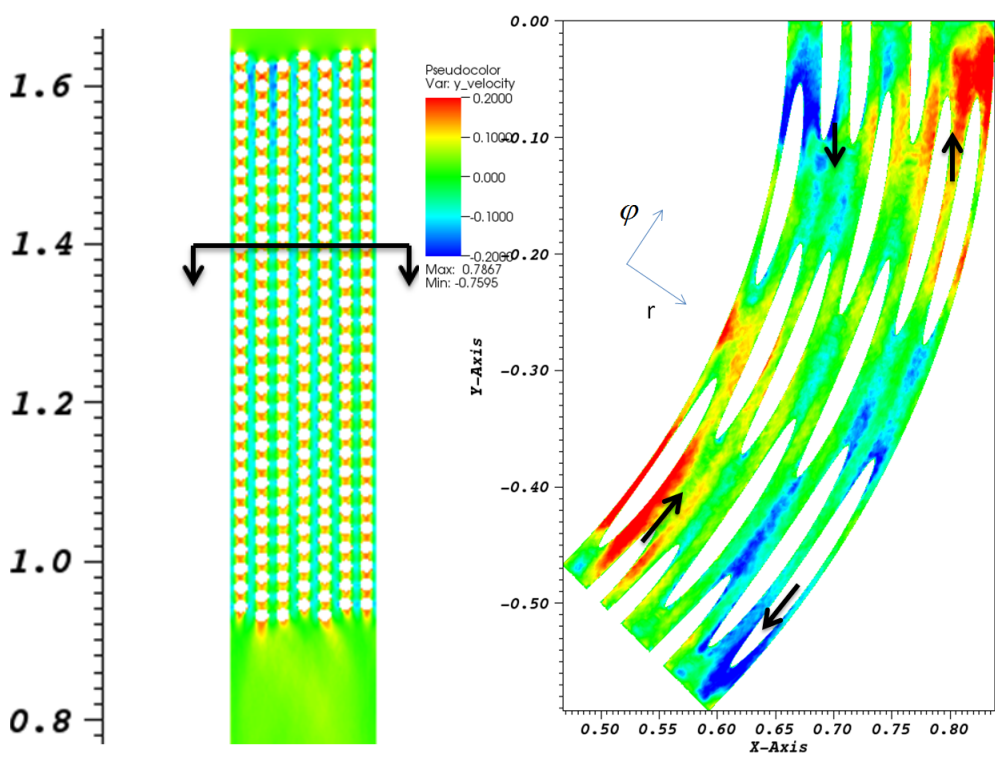

(a)

(b)

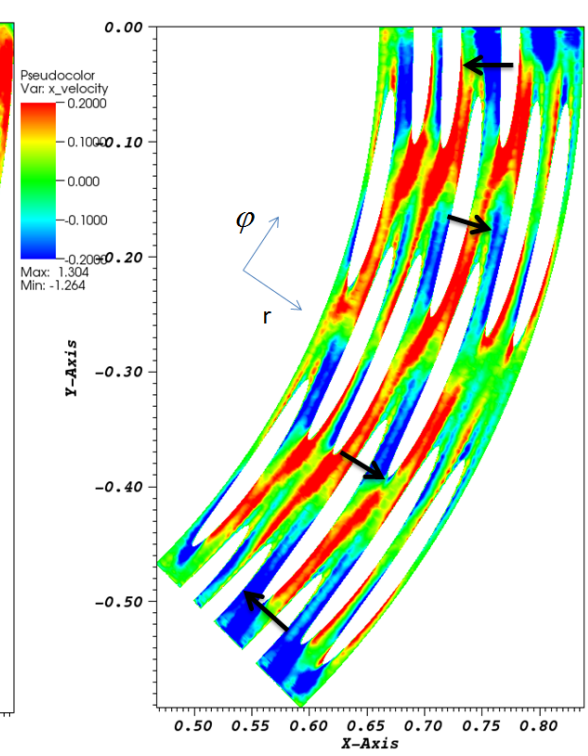

(c)

Fig. 5.18. Average velocity snapshot in axial cross section $(Z=1.4 \mathrm{~m}$, flow goes downward). a) cross section location, b) azimuthal velocity, c) radial velocity.

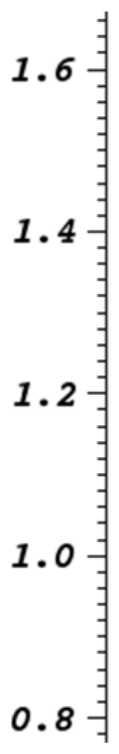

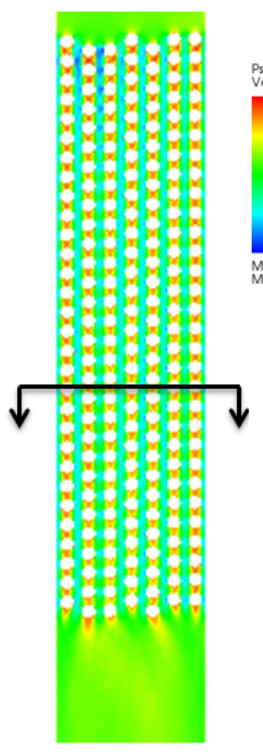

(a)

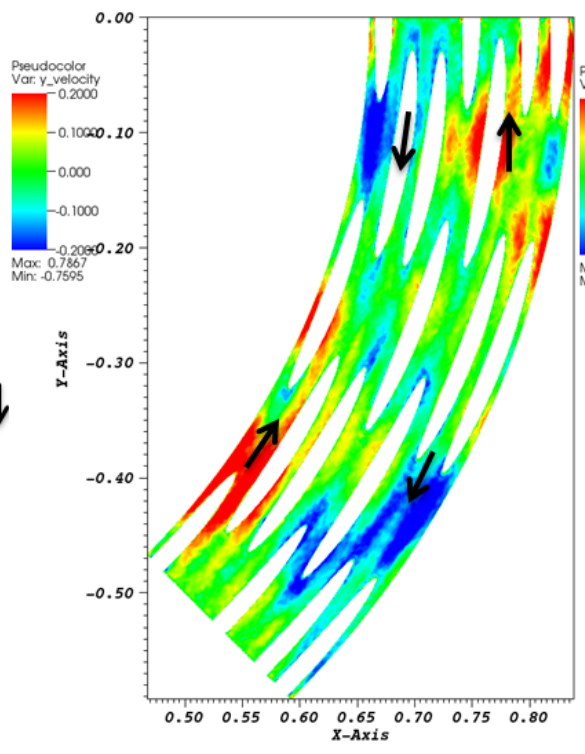

(b)

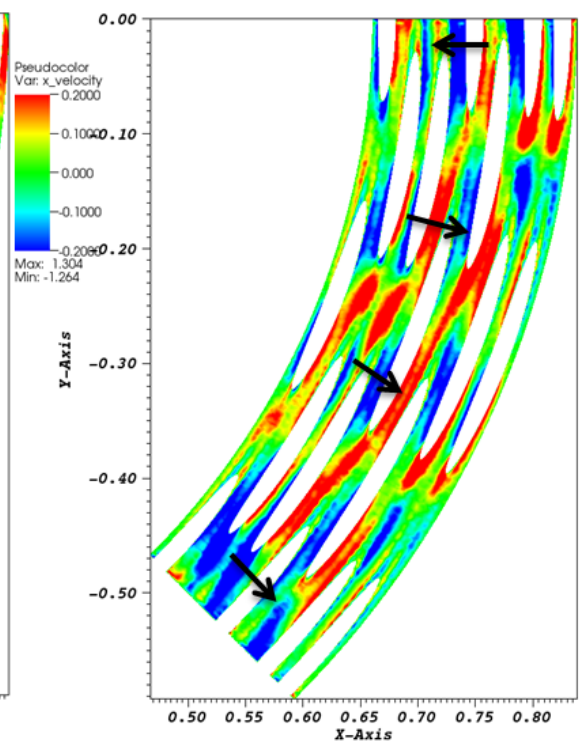

(c)

Fig. 5.12. Average velocity snapshot in axial cross section $(Z=1.2 \mathrm{~m}$, flow goes downward). a) cross section location, b) azimuthal velocity, c) radial velocity. 

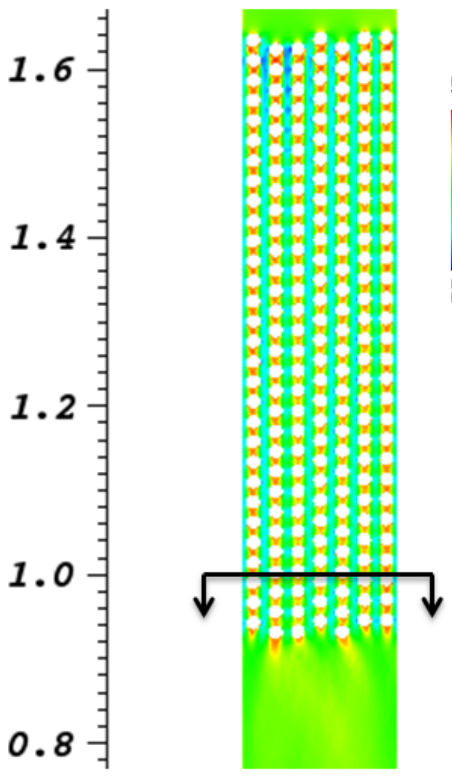

(a)

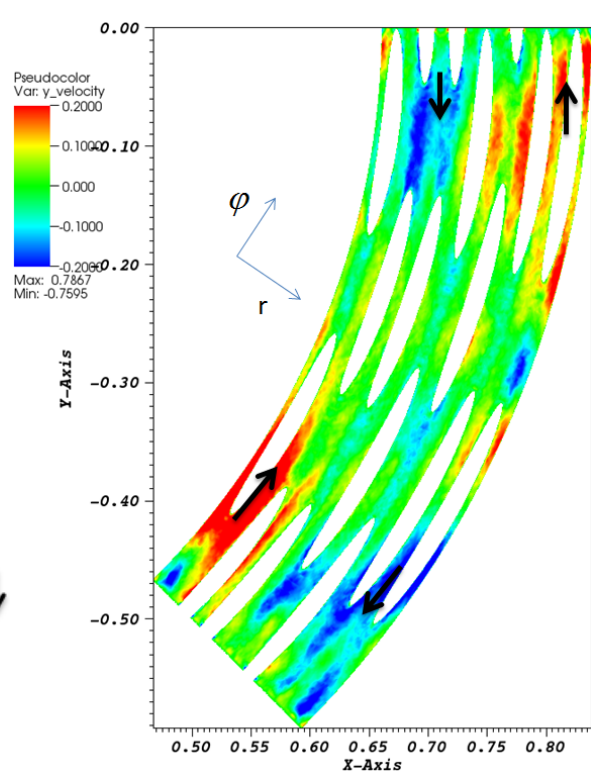

(b)

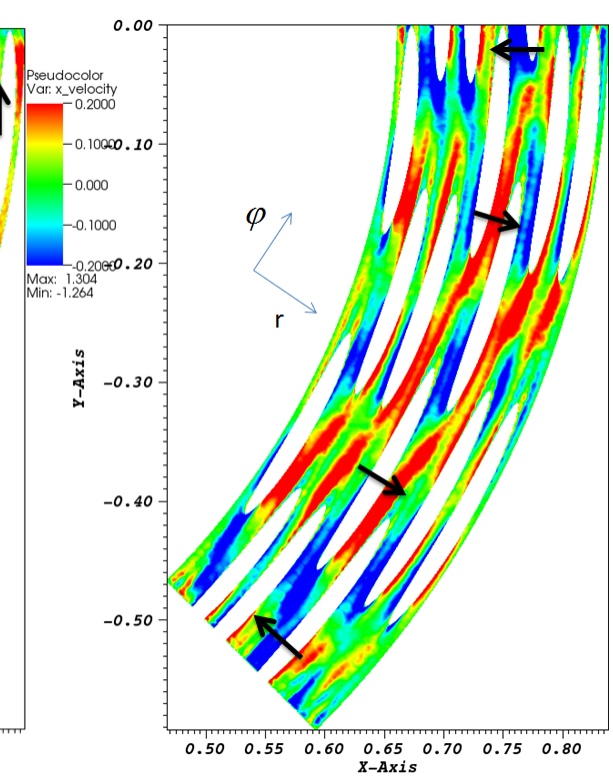

(c)

Fig.5.13. Average velocity snapshot in axial cross section $(Z=1.0 \mathrm{~m}$, flow goes downward $)$. a) cross section location, b) azimuthal velocity, c) radial velocity.

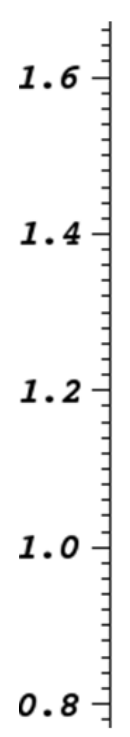

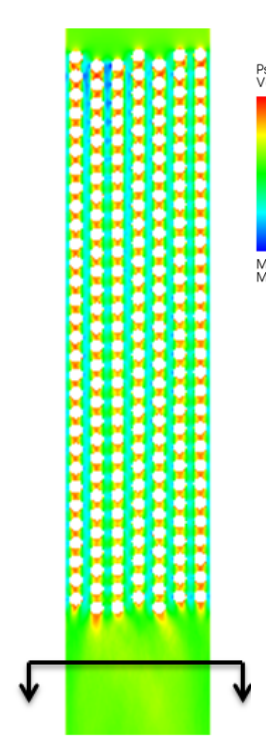

(a)

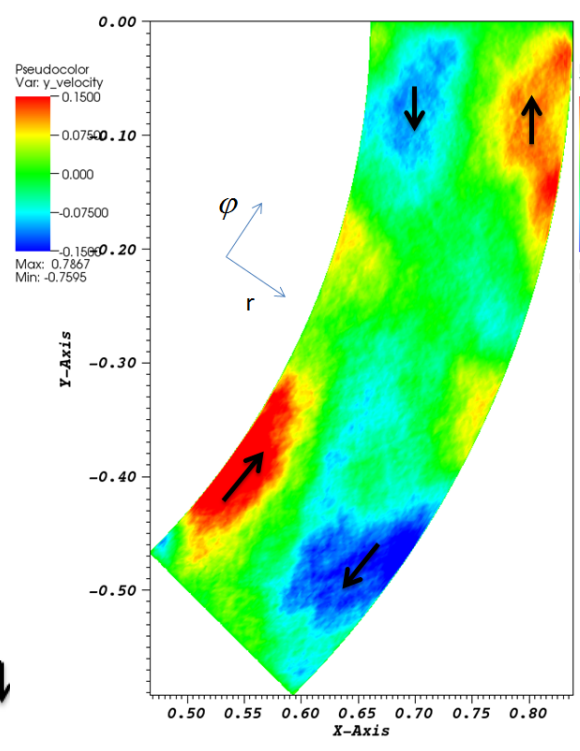

(b)

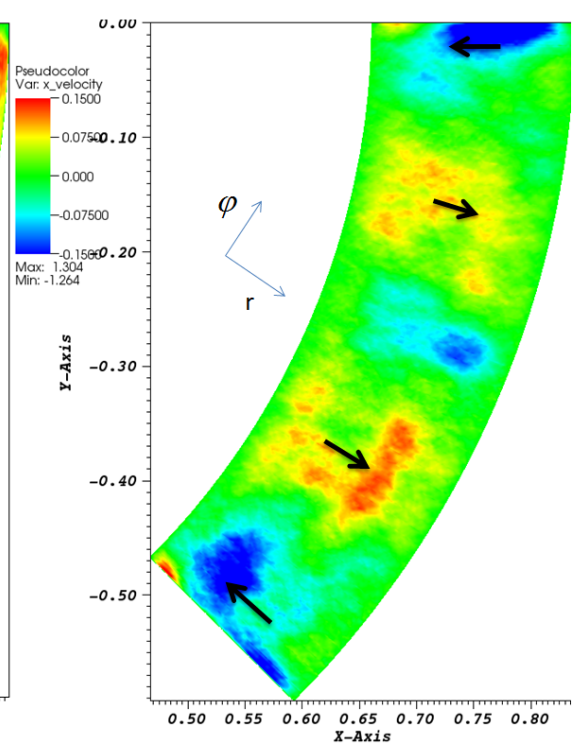

(c)

Fig. 5.14. Average velocity snapshot in axial cross section $(Z=0.85 \mathrm{~m}$, flow goes downward). a) cross section location, b) azimuthal velocity, c) radial velocity. 


\subsubsection{Structural Simulations}

The structural modeling of steam generators presents a number of challenges. Foremost is the modeling of the joints connecting the tubes to the support structures. The connections may take a number of forms, from completely bolted joints to hanger arrangements where the connection restrains the tubes only because of the effect of gravity.

Diablo is a nonlinear structural mechanics code with sophisticated contact models. In particular, Diablo can accurately model contact, release, and gross sliding. Especially in the case of hanger arrangements, the ability to model this behavior is crucial in some situations, as when the bars are subject to gross motion. In these cases typically Coulomb-type friction models are appropriate for modeling the dissipation of the joints.

In situations of high-frequency but small-amplitude vibration, bolted joints often are a source of significant dissipation in the system. This dissipation is traceable to frictional processes under conditions of microslip within the joints. Traditional large-Coulomb models such as employed by Diablo generally require very meshes and small (but still nonlinear) time steps in order to resolve the microslip adequately. Instead, one may utilize semi-empirical interface models [18], [19], [20], [23]. These models are based on the mathematical theory of hysteresis formulated by Preisach, and may take a number of equivalent forms [21], [22]. Currently Diablo does not have any of these models implemented, but they fit within the general framework of Diablo's contact infrastructure. Besides modeling the dissipation of a joint, these models can account for the (varying) stiffness of the joint itself. Note that the behavior of joints in dry conditions is generally different from that in wet conditions.

In a one-way coupled calculation, the structural calculation needs to approximate the feedback effect due to the effect of the motion of the structure on the surrounding fluid, which modifies the fluid traction applied to the structure. To first order, this effect is typically modeled as "added mass", "added damping", and in some cases "added stiffness", see [24],[25],[26], [27]. In the simplest implementation, one augments the mass and damping of the heat exchanger tubes to account for the presence of the fluid. This is obviously a first-order approximation, and it falls apart completely at high flow rates where the tube arrays are subject to fluid-elastic instability. Even at flow rates lower than the fluid-elastic stability limit, the effective added mass and damping may vary as a function of the flow velocity, as in [34].

Ultimately, in order to account for the added mass effect, additional mass-per-unit-length is added to the tubes. In order to account for the added damping, in addition to structural damping that is not directly modeled (e.g. at the joints), Rayleigh Damping is used for the tubes, which consists of mass- and stiffness- proportional terms.

In order to evaluate the structural approximation, a modal analysis was performed on a subset of the problem consisting of a single tube array along with the entire support structure. The support structure was modeled as four solid panels of structural steel, though in reality the support structure consists of hanger bars for which no additional information is available. The material properties of the support structure were varied in order to simulate different states of connection between the support structures and the hanger bars.

Chen et al. [7] performed a number of different experiments in order to establish the frequency response of the system in air. Figures 5.15 and Fig. 5.16 report results of some of these experiments. Typical mode shapes have "nodes" at the hanger bars, as in Fig. 5.17. According to [7], the frequency response is 
best characterized by bands of frequency where the most significant response is expected to occur. These are reported in [7] on page 550 as follows:

"The upper and lower bounds of the frequency bands were selected as follows: column 16, 153-212 Hz; 15B, 175-232 Hz; 15A, 196-249 Hz; 14B, 219-266 Hz; 14A, 239-282 $\mathrm{Hz} ; 13 \mathrm{~B}, 251-313 \mathrm{~Hz} ; 13 \mathrm{~A}, 223-315 \mathrm{~Hz}$."

It is noted that clamping conditions were not that important, as:

"The effect of clamping is small. The only significant effect is to increase the coupling between column $16 \mathrm{~A}$ and $15 \mathrm{~B}$; the frequency bands for each column are practically the same as the unclamped case"

The results of our modal analysis with high stiffness but low mass supports are included as Fig. 5.18. In this case the supports had the material properties of solid steel, but with zero mass (as if they were hollow bars). The supports were constrained at the top and bottom and restrained from moving radially along each vertical edge. The natural frequency of the first mode is $181 \mathrm{~Hz}$, somewhat higher than the expected value of $\sim 150 \mathrm{~Hz}$.

Numerical experiments investigated the effect of the stiffness of the supports. If the stiffness of the hanger bars was reduced by a factor of 1000 , but the boundaries of the supports were fixed all the way around, then the natural frequency of the first mode could be reduced to approximately $103 \mathrm{~Hz}$, as in Fig. 5.19 A full system modal analysis demonstrated that these supports were too soft, as the first mode ceases to be a tube mode but instead becomes a mode of deformation of the supports themselves. Hence, subsequent analyses utilized the initial $(181 \mathrm{~Hz}$ first mode in air) support arrangements. A more complete analysis would include the actual support structures themselves, with a sufficiently detailed model of the joint between the supports and the tubes.

The experiments presented in [7] included vibration analysis in standing water. It was found that on average the natural frequency of the tubes was reduced by a factor around 1.214, as in Fig. 5.20, which corresponded to an "added mass coefficient" of 0.805 , see page 550 of [7]. To investigate our correct use of added mass, we performed a modal analysis as in Fig. 5.18, but with added mass per unit length attached to the tube, equivalent to 1.85 times the density of the original tube. This should reduce the first mode's natural frequency by the factor $\operatorname{sqrt}(1.85)=1.36$.

The results are included in Fig. 5.21, where the first mode is now $\sim 133 \mathrm{~Hz}$, giving a frequency ratio of $180.96 / 133.1=1.36$, and confirming our calculations. Subsequent full-system models utilized an added mass ratio of 1.47 , which provides a frequency ratio of $(1.214)^{2}=1.47$. Thus the first mode of the full system model is expected to be $180.96 / 1.214=149 \mathrm{~Hz}$, higher than the expected value of around 115 Hz. Again, without modeling the supports with more detail, this was judged the best compromise; however, other choices can easily be rerun in order to establish sensitivity. 
TABLE 3

Natural frequencies from steady-state excitations (in $\mathrm{Hz}$ )

\begin{tabular}{cc}
\hline $\begin{array}{c}\text { Exciter at tube } \\
16-1\end{array}$ & $\begin{array}{c}\text { Exciter at tube } \\
16-17\end{array}$ \\
\hline- & $105 \cdot 7$ \\
- & $117 \cdot 8$ \\
- & $141 \cdot 2$ \\
$152 \cdot 9$ & $150 \cdot 2$ \\
$156 \cdot 8$ & $155 \cdot 9$ \\
$162 \cdot 9$ & $161 \cdot 5$ \\
$169 \cdot 2$ & $170 \cdot 0$ \\
$173 \cdot 2$ & $174 \cdot 5$ \\
- & $176 \cdot 0$ \\
$178 \cdot 9$ & $179 \cdot 0$ \\
$185 \cdot 7$ & $186 \cdot 6$ \\
- & $190 \cdot 8$ \\
$194 \cdot 4$ & - \\
$198 \cdot 2$ & - \\
$202 \cdot 4$ & - \\
$206 \cdot 3$ & - \\
$210 \cdot 3$ & $210 \cdot 0$ \\
\hline
\end{tabular}

Fig. 5.15. - Table 3 from [7], Measured response of tubes in column 16 in air.

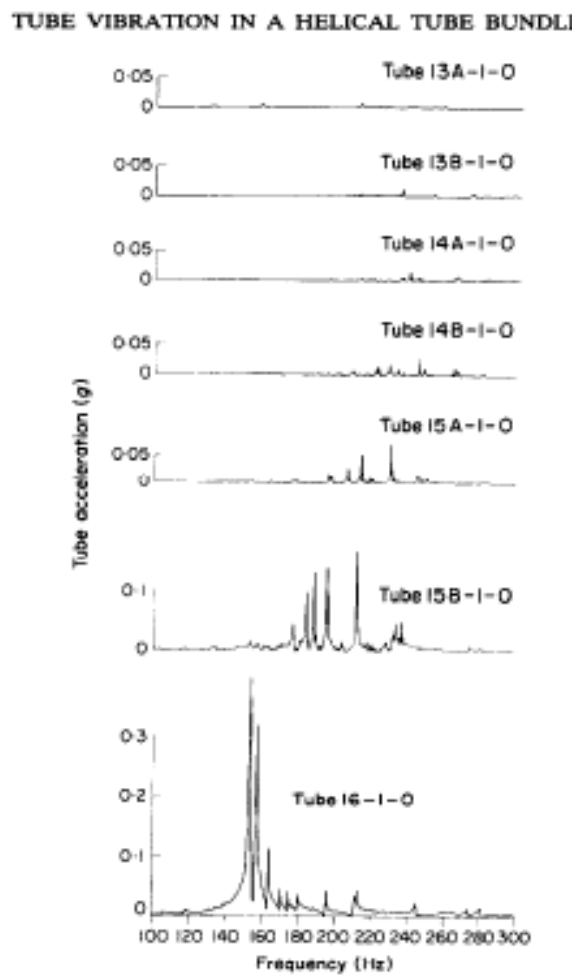

Figure 7. Tube responses to a steady state excitation at tube 16-1.

Fig. 5.16 - Figure 7 from [7], Response spectra of various tubes due to excitation at tube 16-1. 
TUBE VIBRATION IN A HELICAL TUBE BUNDLE

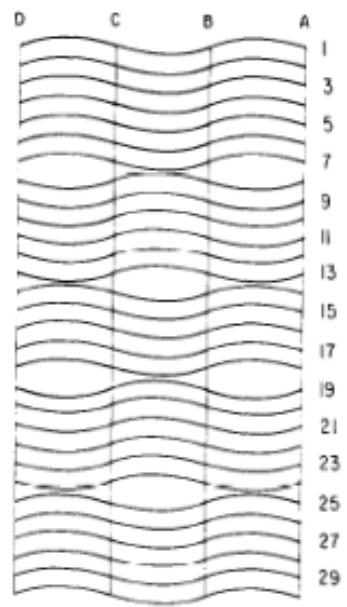

Figure 5. Tube mode shapes at $152.9 \mathrm{~Hz}$ in the out-of-plane direction of Column 16 in air.

Fig. 5.17 - From [7]. Typical mode shapes at $152.9 \mathrm{~Hz}$ in out-of-plane direction for Column 16 in air.

DB: dblsprplt.mili

Cycle: 0 Time: 180.916

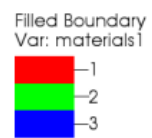

Pseudocolor

Var: evec_disp_magnitude

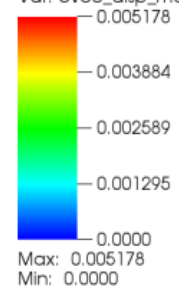

Z
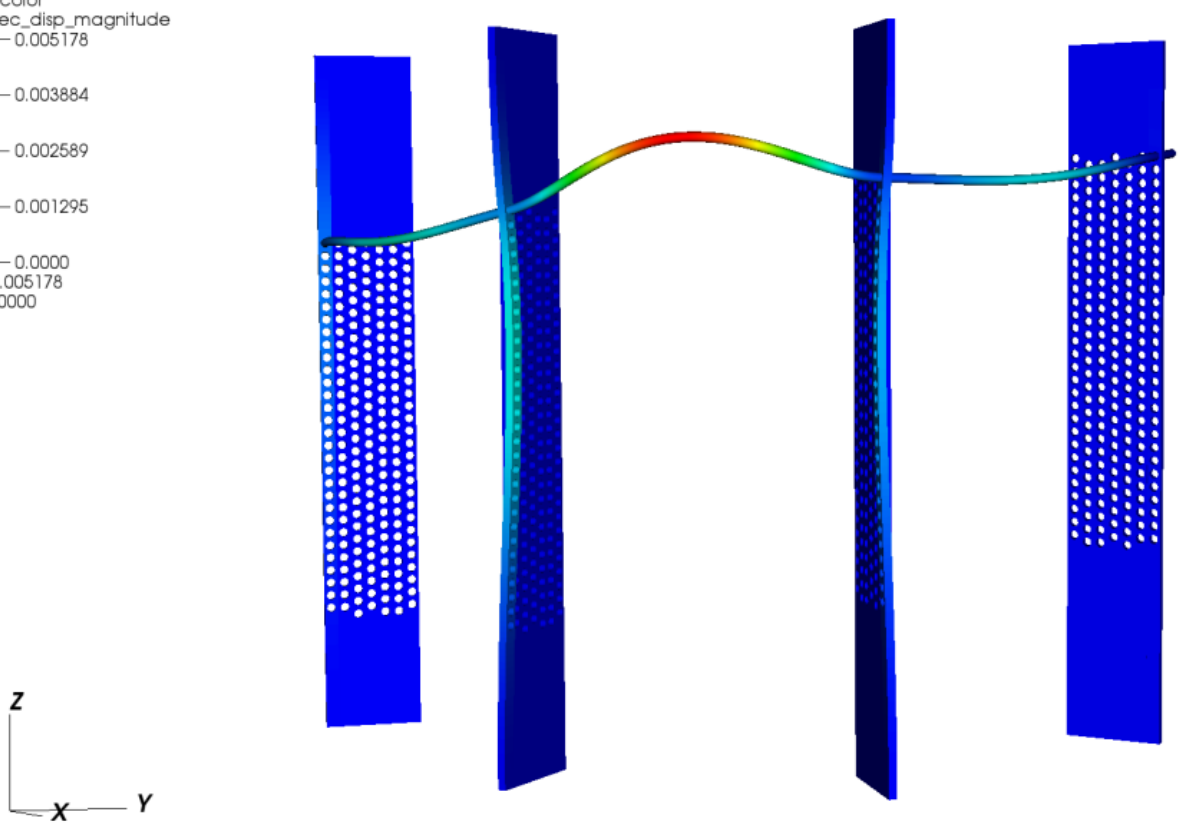

Fig. 5.18 - First mode (181 Hz) of tube 16-1 using high stiffness but zero density supports. 


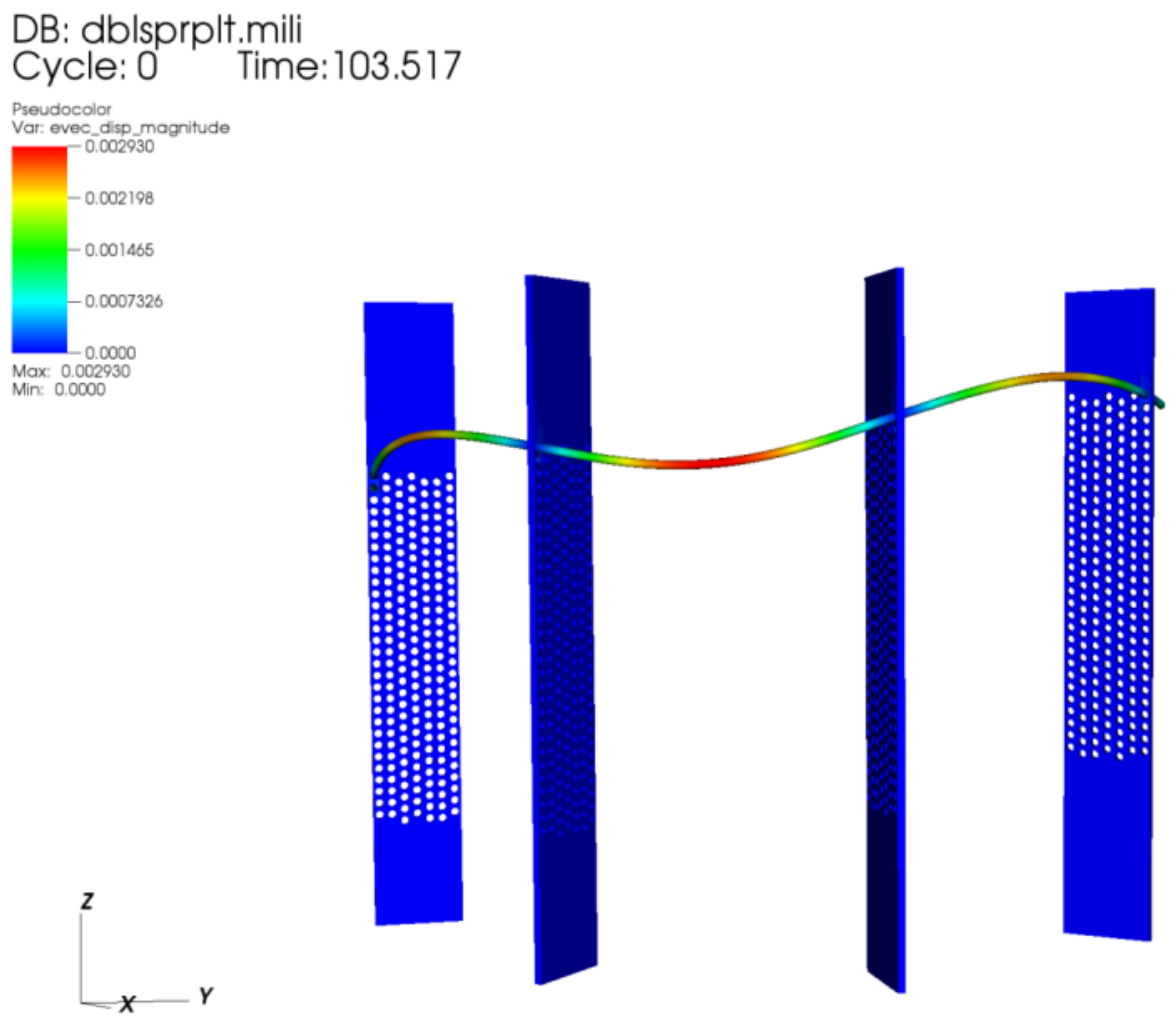

Fig. 5.19 - First mode $(103 \mathrm{~Hz})$ of tube 16-1 using low stiffness, zero density, highly constrained supports.

TABLE 5

Natural frequencies in air and in water

\begin{tabular}{|c|c|c|c|c|c|c|}
\hline \multicolumn{3}{|c|}{$\begin{array}{l}\text { Natural frequency } \\
\text { in air }\left(f_{a}\right)(\mathrm{Hz})\end{array}$} & \multicolumn{3}{|c|}{$\begin{array}{l}\text { Natural frequency } \\
\text { in water }\left(f_{w}\right)(\mathrm{Hz})\end{array}$} & \multirow[b]{2}{*}{$f_{a} / f_{w}$} \\
\hline $16 \mathrm{~A}-27$ & $16 \mathrm{~A}-1$ & $\begin{array}{c}\text { Average } \\
f_{a}\end{array}$ & $16 \mathrm{~A}-27$ & $16 \mathrm{~A}-1$ & $\begin{array}{c}\text { Average } \\
f_{w}\end{array}$ & \\
\hline $140 \cdot 0$ & $140 \cdot 0$ & $140 \cdot 0$ & $115 \cdot 0$ & $115 \cdot 6$ & $115 \cdot 3$ & $1 \cdot 218$ \\
\hline $150 \cdot 2$ & $150 \cdot 2$ & $150 \cdot 2$ & 123.0 & 123.0 & $123 \cdot 0$ & 1.221 \\
\hline 155.9 & 155.6 & 155.8 & 128.5 & 127.6 & 128.0 & 1.217 \\
\hline 161.5 & 161.4 & 161.5 & 135.0 & 134.5 & 134.8 & 1.198 \\
\hline
\end{tabular}

Average $\left(f_{a} / f_{w}\right)=1 \cdot 214$

Fig. 5.20 - Natural frequencies in air and water of tube 16-1 and 16-27, from [7]. 


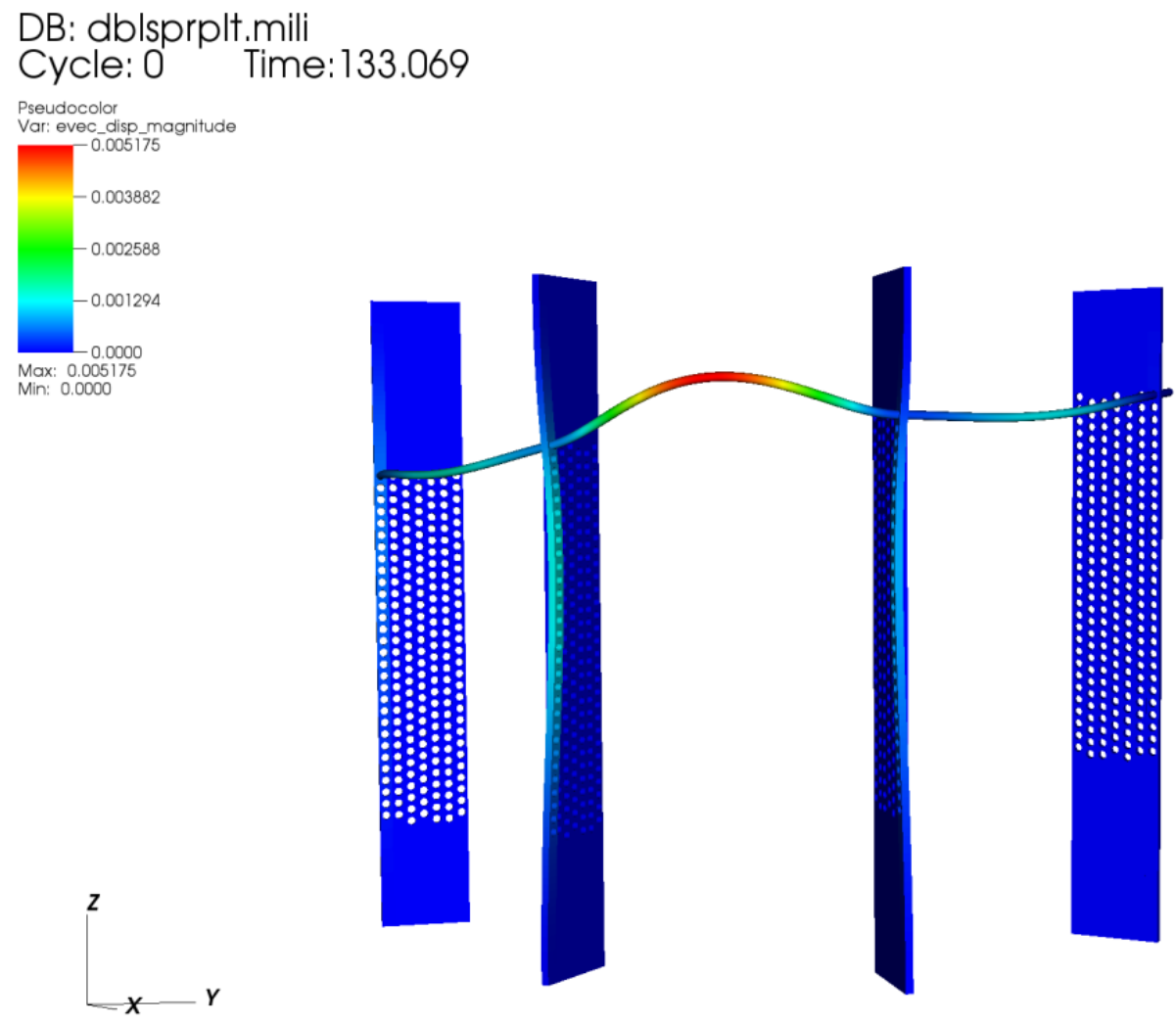

Fig. 5.21. - First mode (181 Hz) of tube 16-1 using high stiffness but zero density supports, mass ratio of 1.85 versus the standard tubes

\subsubsection{Coupled Calculations}

We performed a one-way coupled analysis using the full-system model, with the added mass ratio of 1.47 as explained in the previous section. Chen et al. [7] measured the damping characteristics of the system in still water as ranging between $0.5 \%$ and $1.44 \%$; these are reported in Fig. 5.22. Chen et al. [7] also measured damping as a function of flow velocity for tube 16-1, reported in Fig. 5.23.. Note that the damping increases as a function of flow velocity, and that above approximately $1.75 \mathrm{~m} / \mathrm{s}$ the behavior is erratic.

For each different flow velocity $(1.28 \mathrm{~m} / \mathrm{s}$ and $3.33 \mathrm{~m} / \mathrm{s}$ gap velocity), we performed two analyses using Rayleigh damping, chosen to be $0.75 \%$ and $1.5 \%$, centered between $100 \mathrm{~Hz}$ and $200 \mathrm{~Hz}$. The full system model contained 3,691,464 nodes and 3,595,200 elements. We initialized the problem statically using a time-averaged value of the pressure. The dynamic analysis then began from the static displacement.

The supports utilized 8-node hexahedral elements employing "B-bar" reduced integration. The tubes used 4-node Hughes-Liu shell elements. Over 1,500 time sequences mapped from Nek5000 at time steps of 1.0e-4 were loaded into Diablo. Diablo itself utilized time steps of 5.0e-5, and implicit Newmark time integration with factors beta $=0.25$ and gamma $=0.5$. The Diablo calculations were performed on the Lawrence Livermore National Laboratory machine "cab", on 256 nodes, 1 processor-per-node, using the direct solver library PWSMP. Though the analysis can continue, the initial analyses all consisted of 
slightly over 1,800 time steps (different simulations completed slightly different numbers of time steps, generally in the range of 18,34 to 18,28 ), corresponding to 0.09 seconds of simulation time.

TABLE 6

Damping of column 16 in water

\begin{tabular}{|c|c|c|}
\hline Excitation & $\begin{array}{c}\text { Natural } \\
\text { frequency } \\
(\mathrm{Hz})\end{array}$ & $\begin{array}{c}\text { Damping } \\
(\%)\end{array}$ \\
\hline \multirow[t]{2}{*}{$16-1-0$} & $129 \cdot 4$ & 0.76 \\
\hline & $(115 \cdot 1$ & 1.44 \\
\hline \multirow{3}{*}{$16-27-0$} & $\{123 \cdot 4$ & 0.50 \\
\hline & 128.7 & 0.53 \\
\hline & $134 \cdot 5$ & 0.65 \\
\hline
\end{tabular}

Fig. 5.22 - Damping values in still water, from [7].

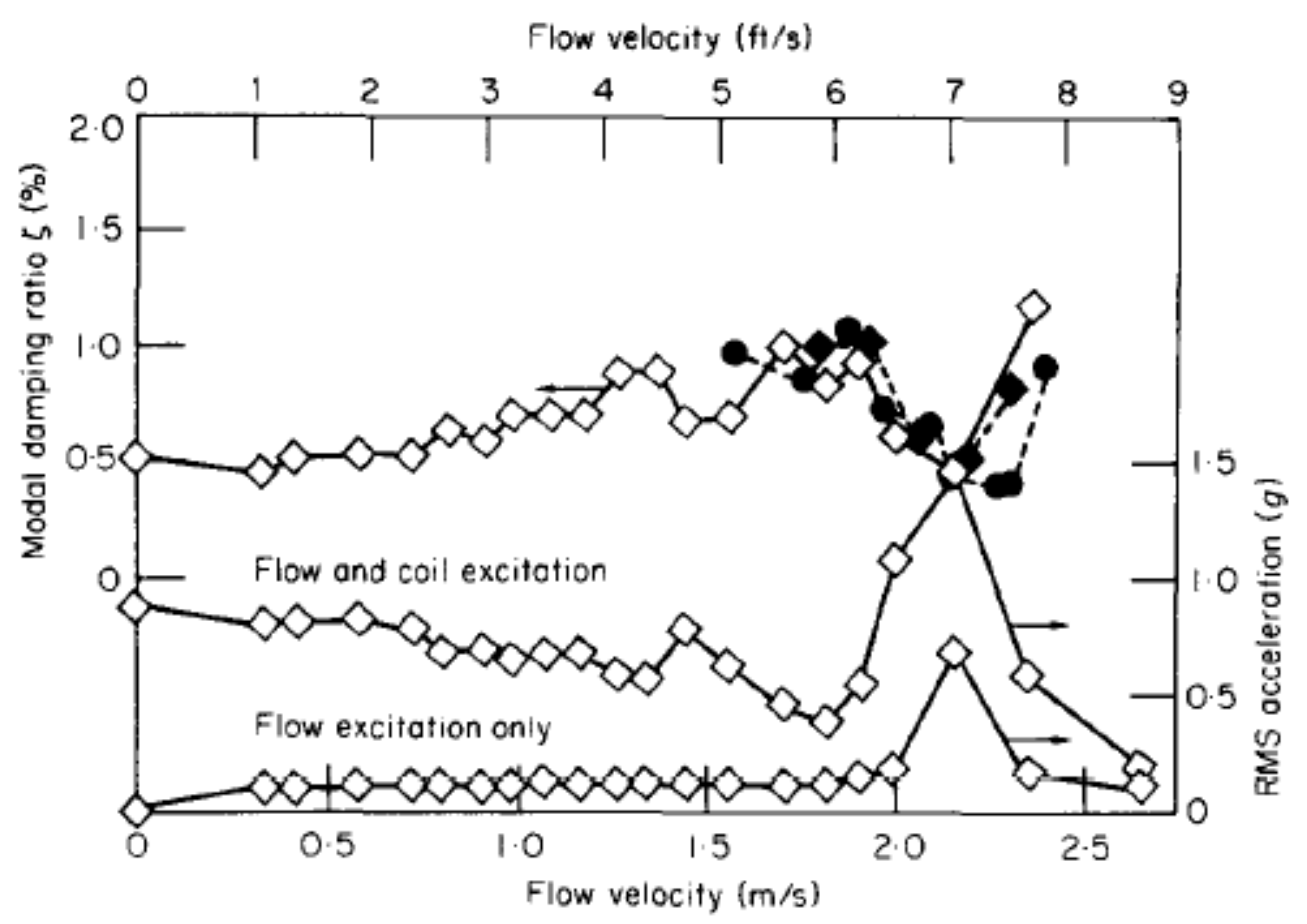

Figure 5.23 - Damping values in flowing water, from [7]. The two bottom curves are the measured RMS tube accelerations as a function of flow excitation only, or flow and coil excitation. It is noted that the tube acceleration decreases with increasing flow velocity below $\sim 1.75 \mathrm{~m} / \mathrm{s}$, commensurate with the increased damping.

Fig. 5.24 reports the results from [7] in terms of RMS tube accelerations. The results for the two simulated flow gap velocities of $1.28 \mathrm{~m} / \mathrm{s}$ and $3.33 \mathrm{~m} / \mathrm{s}$ are transcribed (as best as can be done given the low resolution inherent in the figure) into Tables 5.1, 5.2, 5.3 and 5.4 and compared with the results from Diablo. The positions used for extracting the Diablo output are illustrated by Figures 5.25 and 5.26 which 
also illustrate the static displacements calculated from the time-averaged values of the tube pressures. Accelerations are reported in terms of g's, where $1 \mathrm{~g}=9.81 \mathrm{~m} / \mathrm{s}^{2}$.

At the gap velocity of $1.28 \mathrm{~m} / \mathrm{s}$ the Diablo results bound the accelerations. That is, the $1.5 \%$ damping results have RMS accelerations slightly lower than that from experiment; for example for tube 16-1 one has $0.0952 \mathrm{~g}$ for Diablo versus about 0.14 for the experiment. For the $0.75 \%$ case Diablo has RMS accelerations slightly higher than the experiment; for example Diablo has RMS accelerations of $0.167 \mathrm{~g}$ versus $0.14 \mathrm{~g}$ for the experiment. Note that the results for tube $15 \mathrm{~A}-1$ in the out-of-plane direction appear to be an anomaly - they are significantly higher than the surrounding tubes. This result deserves further investigation; it may represent a local flow condition. No data was reported for this tube in [7] in terms on rms.

At the gap velocity of $3.33 \mathrm{~m} / \mathrm{s}$ the Diablo results do not bound the accelerations. In many cases both the $1.5 \%$ and $0.75 \%$ Diablo cases have RMS accelerations significantly higher than that from the experiment. For example Diablo has RMS accelerations for tube $13 \mathrm{~A}-1$ of $2.07 \mathrm{~g}$ versus $0.2 \mathrm{~g}$ for the experiment. On the other hand, for tube 16-1 Diablo has a maximum RMS tube acceleration of about $4 \mathrm{~g}$, whereas the experiment reports a tube acceleration on the order of $6 \mathrm{~g}$. These results indicate that, for some tubes, such as $13 \mathrm{~A}-1$, the damping is increasing as a function of flow velocity, suppressing the amplitude of the response, as has already been noted in connection with Fig. 5.23. In the case of tube 16-1, it appears that the tube has gone beyond the critical flow velocity, and one-way coupled analyses are no longer valid. This is the same conclusion reached by [7], where the critical flow velocity was calculated to be between 2.8 and $3.2 \mathrm{~m} / \mathrm{s}$, see Fig. 5.27 .

Figure 5.28 reports the results from [7] in terms of frequency spectra. From Fig. 5.28 (a), it is seen that at $1.28 \mathrm{~m} / \mathrm{s}$ flow velocity, the tubes exhibit a wide variety of spectra, with the shorter, stiffer tubes (e.g. 13A$1,14 \mathrm{~A}-1$, etc.) having frequencies generally higher, commensurate with their higher natural frequencies. This behavior is repeated at the gap velocity of $2.70 \mathrm{~m} / \mathrm{s}$. However, at $3.70 \mathrm{~m} / \mathrm{s}$, above the critical flow velocity, the response is uniformly the same frequency of about $120 \mathrm{~Hz}$, with a tight bandwidth. This indicates that the nature of the excitation (and the coupling with the tubes) has changed drastically. Comparisons with the frequency spectra at the $1.28 \mathrm{~m} / \mathrm{s}$ flow velocity are included as Figures 5.29 -5.35. We are generally capturing the frequency content of the signals, with the caveat that as the model of the supports does not have sufficient detail, we generally have higher peak frequencies than the experiment does.

In conclusion, below the critical velocity, for the Argonne SG we can simulate the response of the tubes with good accuracy, assuming that the tube/support interface is modeled sufficiently. Above the critical velocity, the tube/flow interaction appears to require a two-way coupled model. Simulations at a few more flow velocities would be required in order to ascertain whether we can detect the onset of the critical velocity itself with a one-way coupled methodology. 

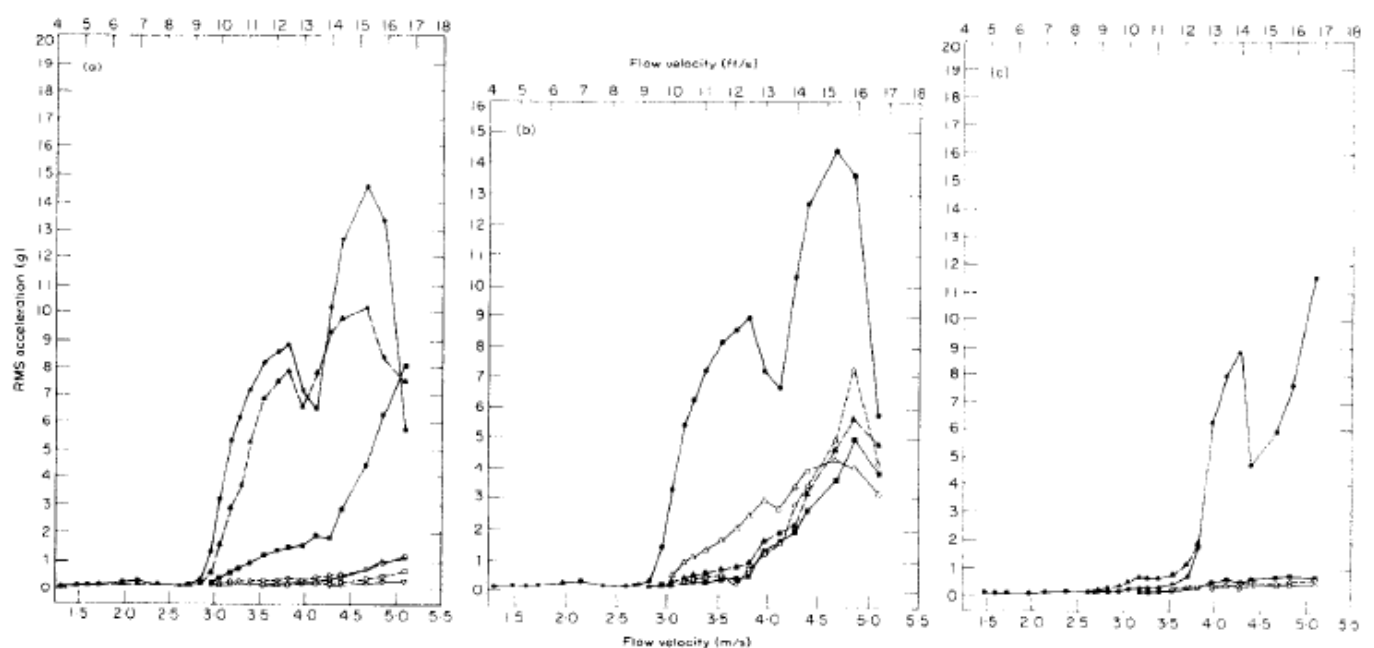

Figure 14. RMS tube accelerations (a) of the upstream tubes, (b) of tubes in column 16 and (c) in the in-plane direction as functions of flow velocity. (a) $-\mathrm{A}--, 15 \mathrm{~B}-1-\mathrm{O} ;--, 15 \mathrm{~A}-1-\mathrm{O} ;--\mathrm{O}--, 14 \mathrm{~B}-1-\mathrm{O} ;-\triangle-, 14 \mathrm{~A}-1-\mathrm{O} ;--\square--, 13 \mathrm{~B}-1-\mathrm{O} ;-\nabla-, 13 \mathrm{~A}-1-\mathrm{O}$; (b)

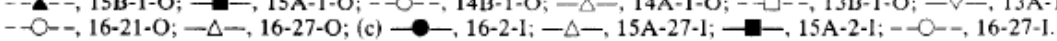

Figure 5.24. - RMS tube accelerations from [7], as a function of tube and flow velocity.

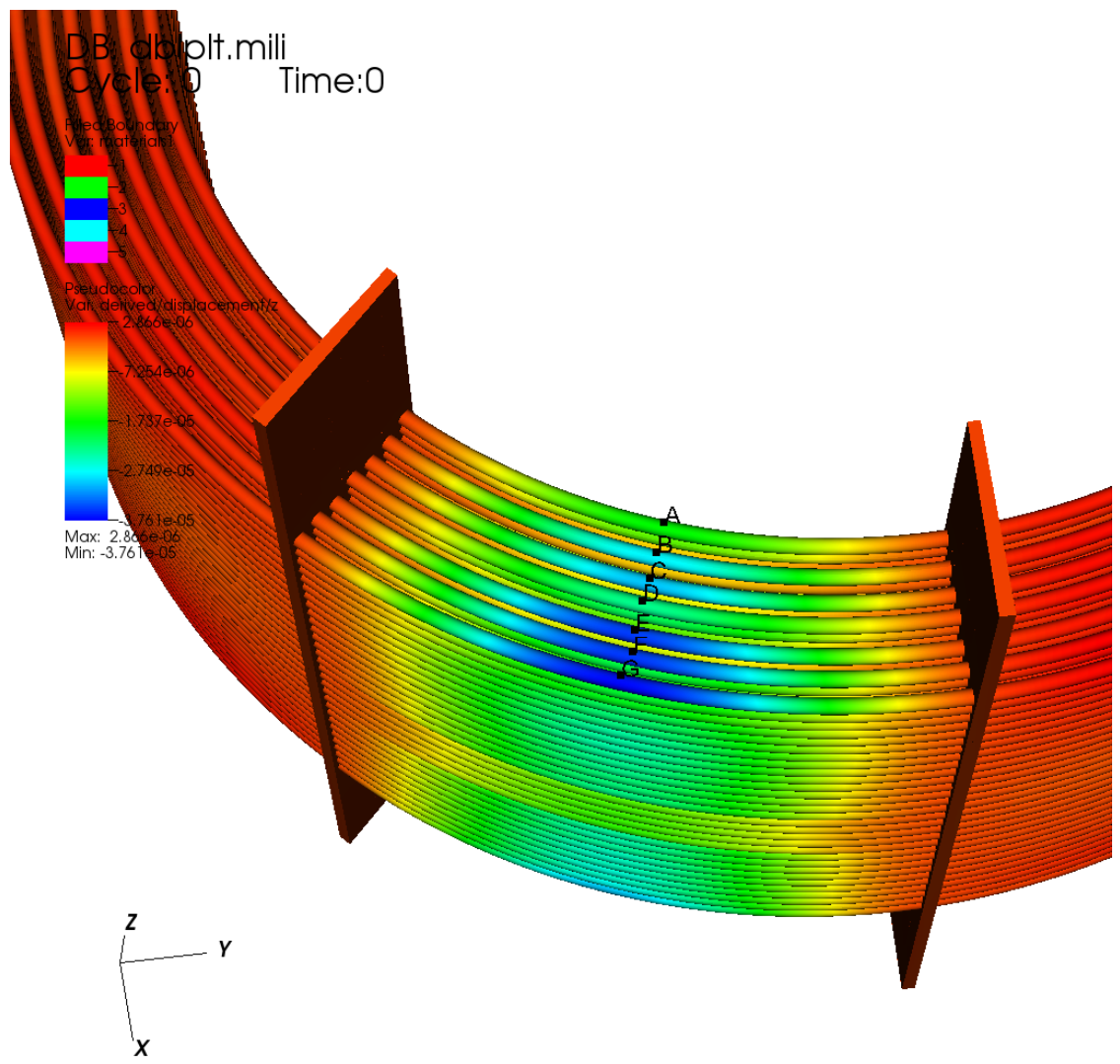

Fig. 5.25. - Positions of the output for tubes $A=13 A-1, B=13 B-1, C=14 A-1, D=14 B-1, E=15 A-1$, $F=15 B-1, G=16-1$. The colors represent the initial static displacement, calculated from the timeaveraged pressure. 


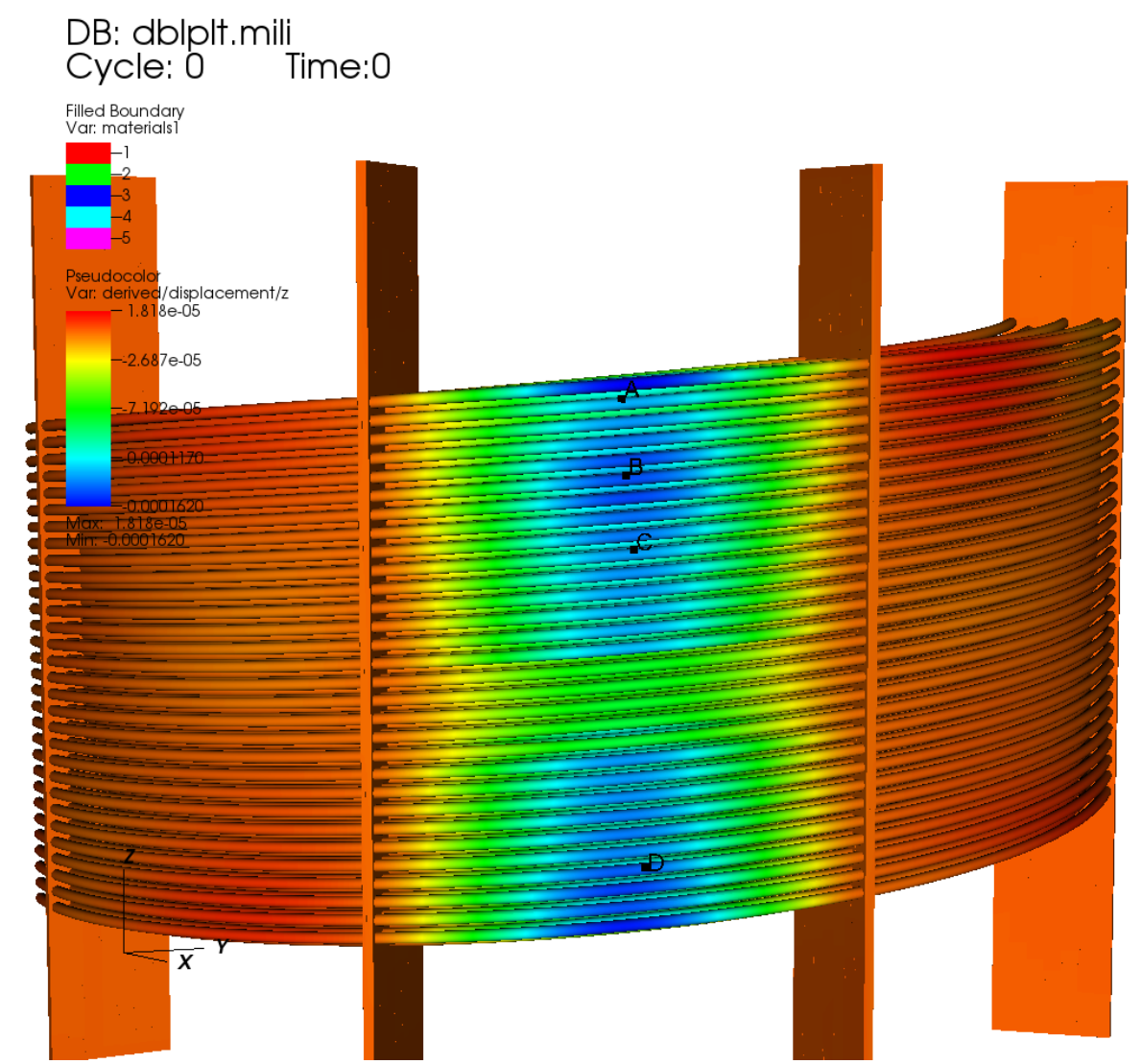

Figure 5.26 - Positions of the output for tubes $A=16-2, B=16-6, C=16-10, D=16-27$. The colors represent the initial static displacement, calculated from the time-averaged pressure.

Table 5.1 - RMS Acceleration Values for Forward-Coupled Argonne SG Calculation, Gap Flow Velocity 1.28mps, Nominal Structural Damping 1.5\%

\begin{tabular}{|l|l|l|l|l|}
\hline $\begin{array}{l}\text { RMS } \\
\text { acceleration }\end{array}$ & $\begin{array}{l}\text { Out- } \\
\text { plane(g) }\end{array}$ & $\begin{array}{l}\text { Chen } \\
\text { paper } \\
\text { est.* }\end{array}$ & In-plane(g) & $\begin{array}{l}\text { Chen } \\
\text { paper } \\
\text { est.* }\end{array}$ \\
\hline $13 \mathrm{~A}-1$ & 0.117 & & 0.101 & \\
\hline $13 \mathrm{~B}-1$ & 0.173 & & 0.123 & \\
\hline $14 \mathrm{~A}-1$ & 0.122 & & 0.116 & \\
\hline $14 \mathrm{~B}-1$ & 0.115 & & 0.144 & \\
\hline $15 \mathrm{~A}-1$ & 0.333 & & 0.0886 & \\
\hline $15 \mathrm{~B}-1$ & 0.107 & 0.14 & 0.114 & \\
\hline $16-1$ & 0.0952 & 0.14 & 0.104 & \\
\hline
\end{tabular}




\begin{tabular}{|l|l|l|l|l|}
\hline $16-2$ & 0.118 & & 0.112 & 0.14 \\
\hline $16-10$ & 0.266 & & 0.17 & \\
\hline $16-27$ & 0.218 & & 0.251 & \\
\hline
\end{tabular}

Table 5.2 - RMS Acceleration Values for Forward-Coupled Argonne SG Calculation, Gap Flow Velocity 1.28mps, Nominal Structural Damping $0.75 \%$

\begin{tabular}{|l|l|l|l|l|}
\hline $\begin{array}{l}\text { RMS } \\
\text { acceleration }\end{array}$ & $\begin{array}{l}\text { Out- } \\
\text { plane(g) }\end{array}$ & $\begin{array}{l}\text { Chen } \\
\text { paper } \\
\text { est.* }\end{array}$ & In-plane(g) & $\begin{array}{l}\text { Chen } \\
\text { paper } \\
\text { est.* }\end{array}$ \\
\hline $13 \mathrm{~A}-1$ & 0.187 & & 0.141 & \\
\hline $13 \mathrm{~B}-1$ & 0.244 & & 0.189 & \\
\hline $14 \mathrm{~A}-1$ & 0.194 & & 0.19 & \\
\hline $14 \mathrm{~B}-1$ & 0.167 & & 0.198 & \\
\hline $15 \mathrm{~A}-1$ & 0.448 & & 0.125 & \\
\hline $15 \mathrm{~B}-1$ & 0.148 & 0.14 & 0.157 & \\
\hline $16-1$ & 0.167 & 0.14 & 0.15 & \\
\hline $16-2$ & 0.149 & & 0.158 & 0.14 \\
\hline $16-10$ & 0.289 & & 0.223 & \\
\hline $16-27$ & 0.265 & & 0.273 & \\
\hline
\end{tabular}

Table 5.3 - RMS Acceleration Values for Forward-Coupled Argonne SG Calculation, Gap Flow Velocity 3.33mps, Nominal Structural Damping $1.5 \%$.

\begin{tabular}{|l|l|l|l|l|}
\hline $\begin{array}{l}\text { RMS } \\
\text { accleration }\end{array}$ & $\begin{array}{l}\text { Out- } \\
\text { plane(g) }\end{array}$ & $\begin{array}{l}\text { Chen } \\
\text { paper } \\
\text { est.* }\end{array}$ & In-plane(g) & $\begin{array}{l}\text { Chen } \\
\text { paper } \\
\text { est.* }\end{array}$ \\
\hline $13 \mathrm{~A}-1$ & 2.08 & 0.2 & 1.66 & \\
\hline $13 \mathrm{~B}-1$ & 2.49 & 0.2 & 1.82 & \\
\hline
\end{tabular}




\begin{tabular}{|l|l|l|l|l|}
\hline $14 \mathrm{~A}-1$ & 2.72 & 0.2 & 1.74 & \\
\hline $14 \mathrm{~B}-1$ & 2.18 & 0.2 & 1.73 & \\
\hline $15 \mathrm{~A}-1$ & 2.66 & 1.0 & 1.61 & \\
\hline $15 \mathrm{~B}-1$ & 1.95 & 4 & 1.75 & \\
\hline $16-1$ & 3.22 & 6 & 1.87 & \\
\hline $16-2$ & 2.71 & & 1.84 & 0.2 \\
\hline $16-10$ & 2.71 & 0.2 & 2.89 & \\
\hline $16-27$ & 2.57 & 0.5 & 3.34 & 0.14 \\
\hline
\end{tabular}

Table 5.4 - RMS Acceleration Values for Forward-Coupled Argonne SG Calculation, Gap Flow Velocity 3.33mps, Nominal Structural Damping 0.75\%.

\begin{tabular}{|l|l|l|l|l|}
\hline $\begin{array}{l}\text { RMS } \\
\text { accleration }\end{array}$ & $\begin{array}{l}\text { Out- } \\
\text { plane(g) }\end{array}$ & $\begin{array}{l}\text { Chen } \\
\text { paper } \\
\text { est.* }\end{array}$ & In-plane(g) & $\begin{array}{l}\text { Chen } \\
\text { paper } \\
\text { est.* }\end{array}$ \\
\hline $13 \mathrm{~A}-1$ & 2.07 & 0.2 & 2.05 & \\
\hline $13 \mathrm{~B}-1$ & 2.94 & 0.2 & 2.39 & \\
\hline $14 \mathrm{~A}-1$ & 3.31 & 0.2 & 2.23 & \\
\hline $14 \mathrm{~B}-1$ & 2.62 & 0.2 & 2.1 & \\
\hline $15 \mathrm{~A}-1$ & 3.8 & 1.0 & 2.37 & \\
\hline $15 \mathrm{~B}-1$ & 2.97 & 4 & 2.45 & \\
\hline $16-1$ & 4.28 & 6 & 2.24 & \\
\hline $16-2$ & 3.41 & & 2.22 & 0.2 \\
\hline $16-10$ & 3.16 & 0.2 & 3.13 & \\
\hline $16-27$ & 2.64 & 0.5 & 3.03 & 0.14 \\
\hline
\end{tabular}


TABLE 8

The critical flow velocity

\begin{tabular}{cccc}
\hline Run & $\begin{array}{c}\text { Critical flow } \\
\text { velocity } \\
(\mathrm{m} / \mathrm{s})\end{array}$ & $\begin{array}{c}\text { Oscillation } \\
\text { frequency } \\
(\mathrm{Hz})\end{array}$ & $\begin{array}{c}\text { Reduced flow } \\
\text { velocity } \\
(\mathrm{V} / \mathrm{fD})\end{array}$ \\
\hline Preliminary run & $2 \cdot 82$ & 124 & $1 \cdot 43$ \\
Run 1 & $2 \cdot 85$ & $123 \cdot 5$ & 1.45 \\
Run 2 & $3 \cdot 32$ & $133 \cdot 5$ & 1.57 \\
Run 3 & $3 \cdot 2$ & 136 & $1 \cdot 48$ \\
Run 4 & $3 \cdot 17$ & 137 & $1 \cdot 46$ \\
\hline
\end{tabular}

Figure 5.27. - The critical flow velocity, from [7].

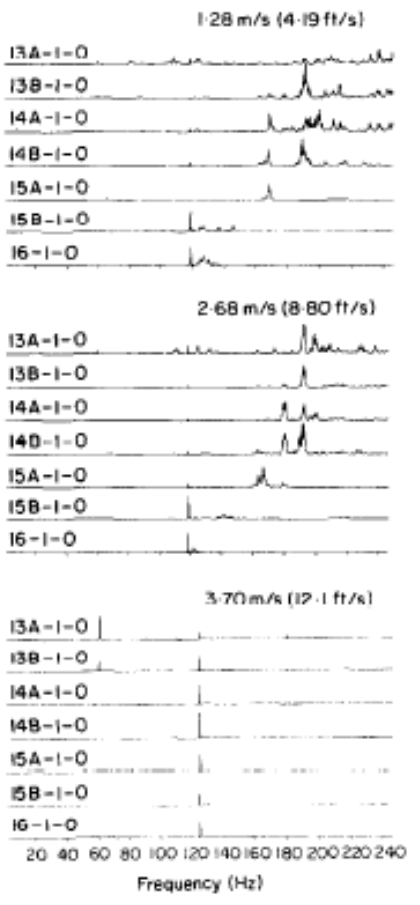

(a)

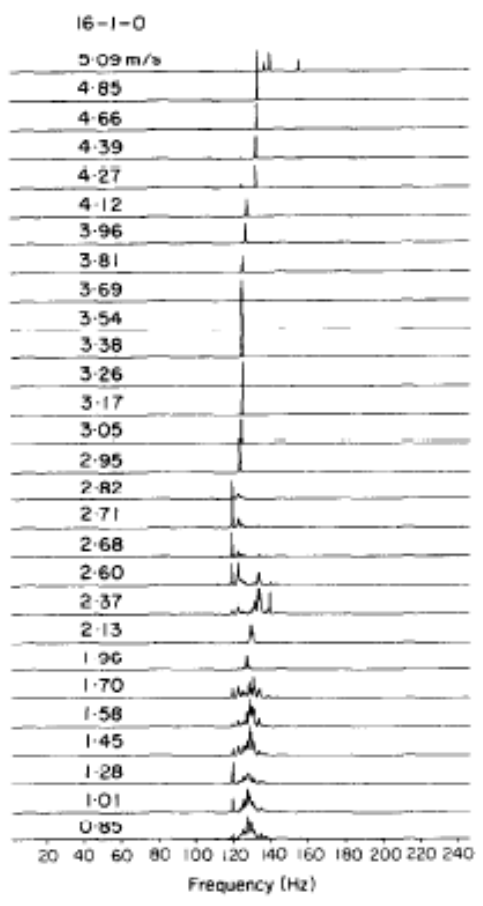

(b)
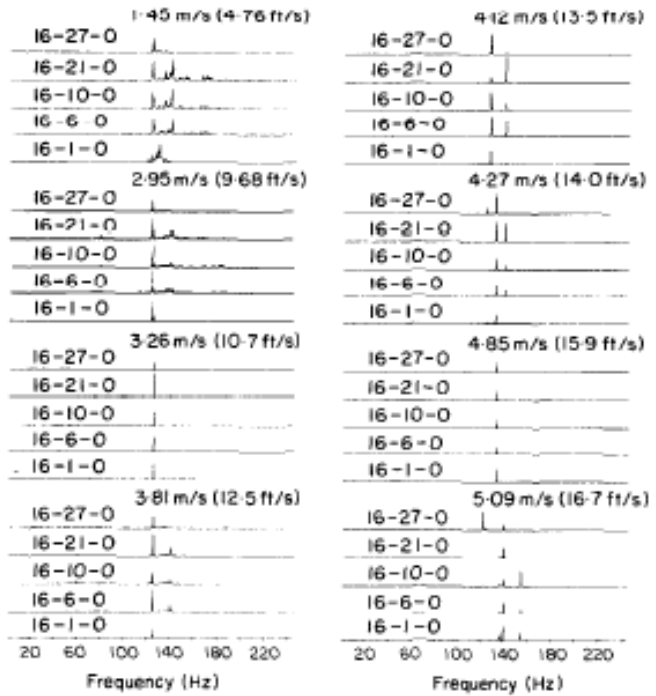

Figure 15. Frequency spectra of tube accelerations (a) in the out-of-plane direction of the upstream tubes; (b) of tube 16-1-O; (c) in the out-of-plane direction of column 16.

Figure 5.28. - Frequency spectra from [7], as a function of tube and flow velocity. 


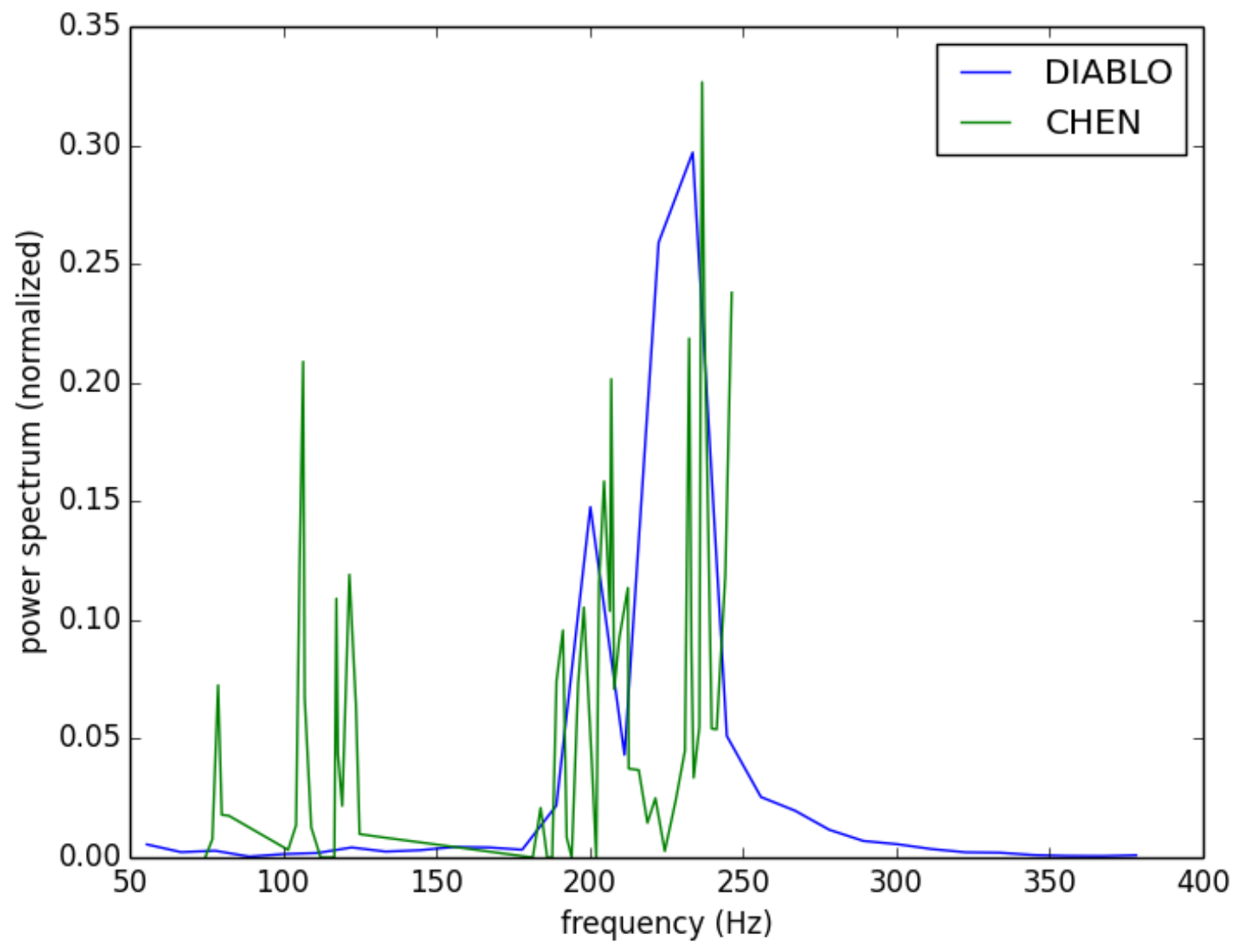

Fig. 5.29 - Comparison of $1.28 \mathrm{~m} / \mathrm{s}$ out-of-plane frequency spectra, tube $13 \mathrm{~A}-1,1.5 \%$ damping. 


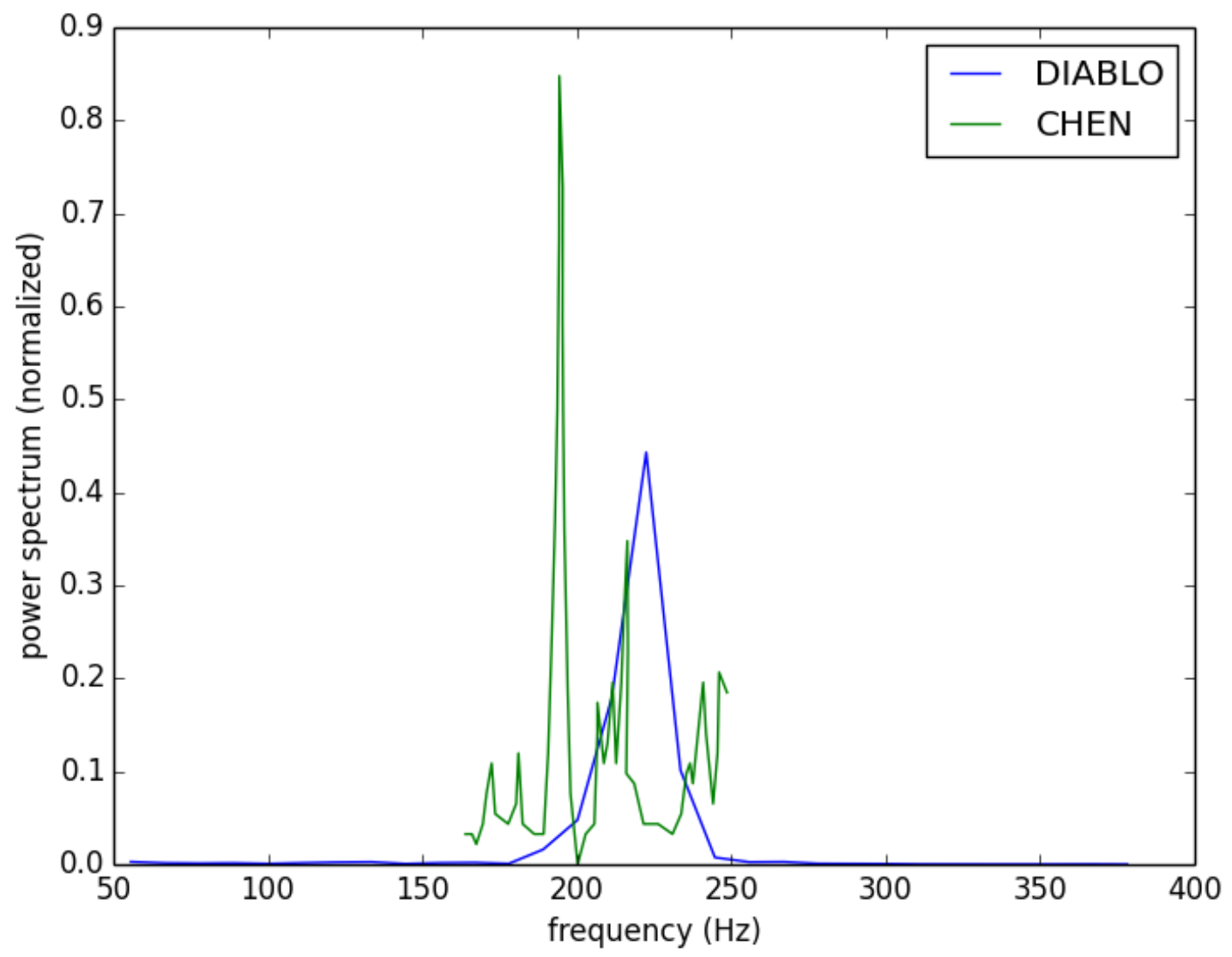

Fig. 5.30. - Comparison of $1.28 \mathrm{~m} / \mathrm{s}$ out-of-plane frequency spectra, tube 13B-1, $1.5 \%$ damping. 


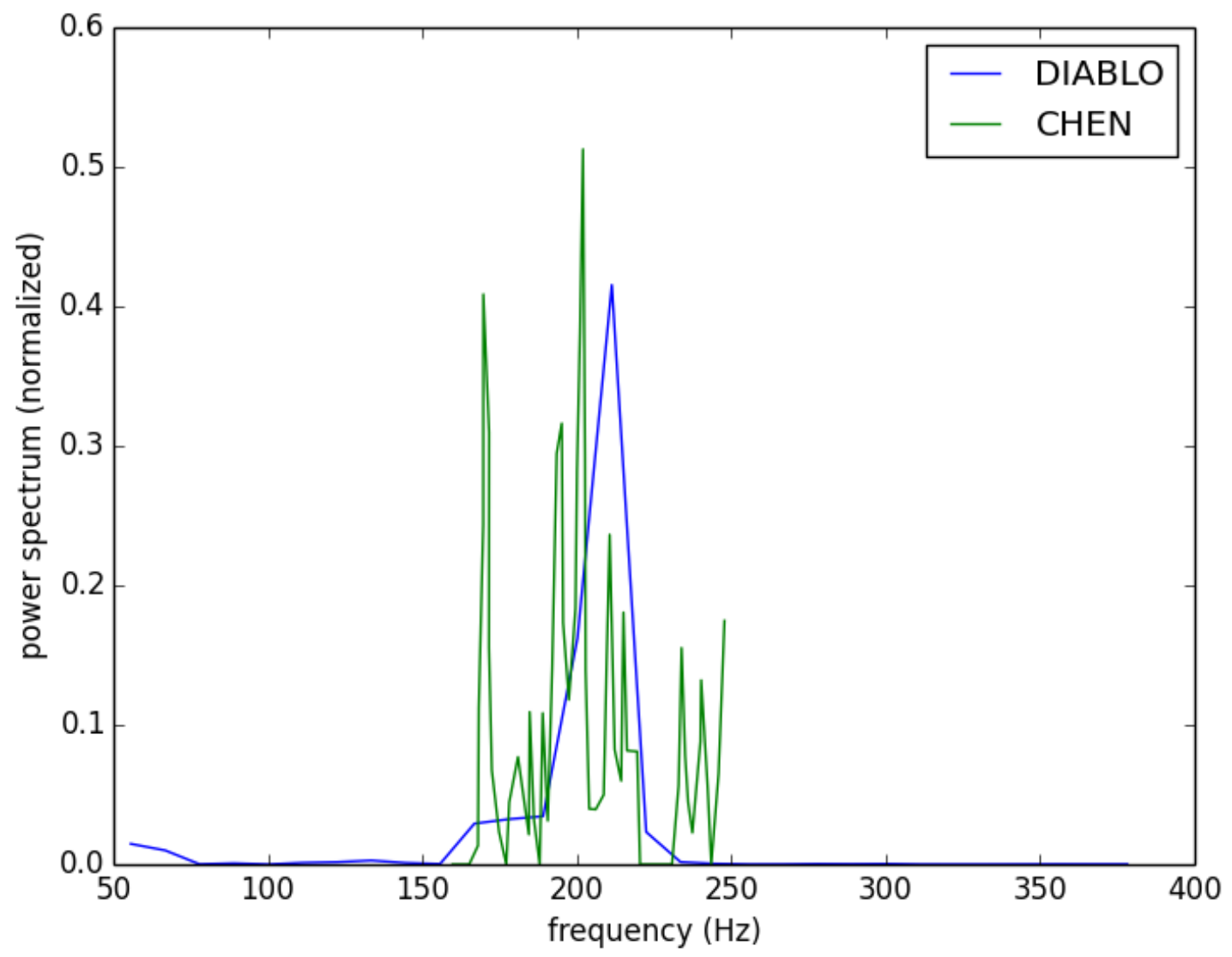

Fig. 5.31. - Comparison of $1.28 \mathrm{~m} / \mathrm{s}$ out-of-plane frequency spectra, tube 14A-1, 1.5\% damping. 


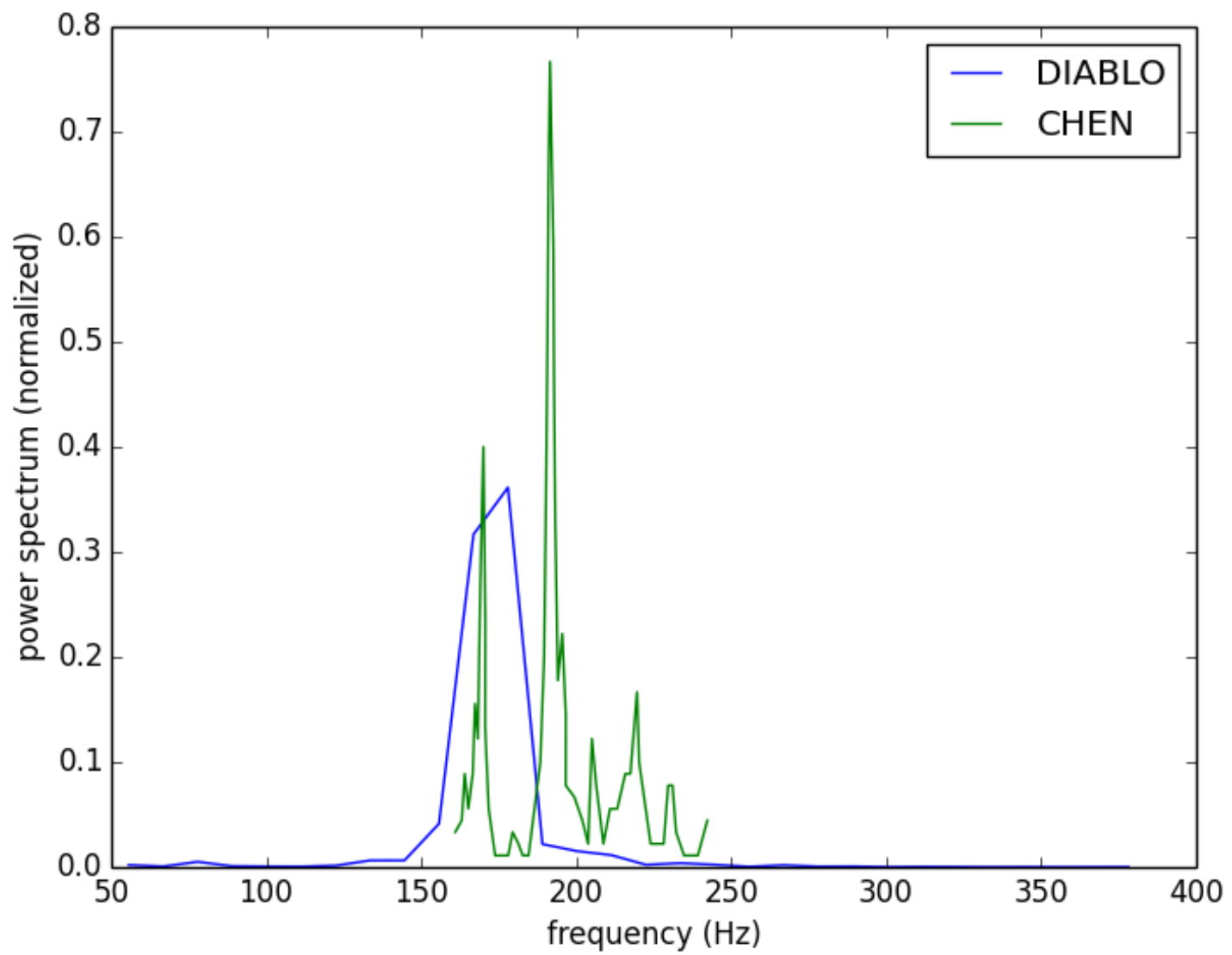

Figure 5.32. - Comparison of $1.28 \mathrm{~m} / \mathrm{s}$ out-of-plane frequency spectra, tube 14B-1, $1.5 \%$ damping. 


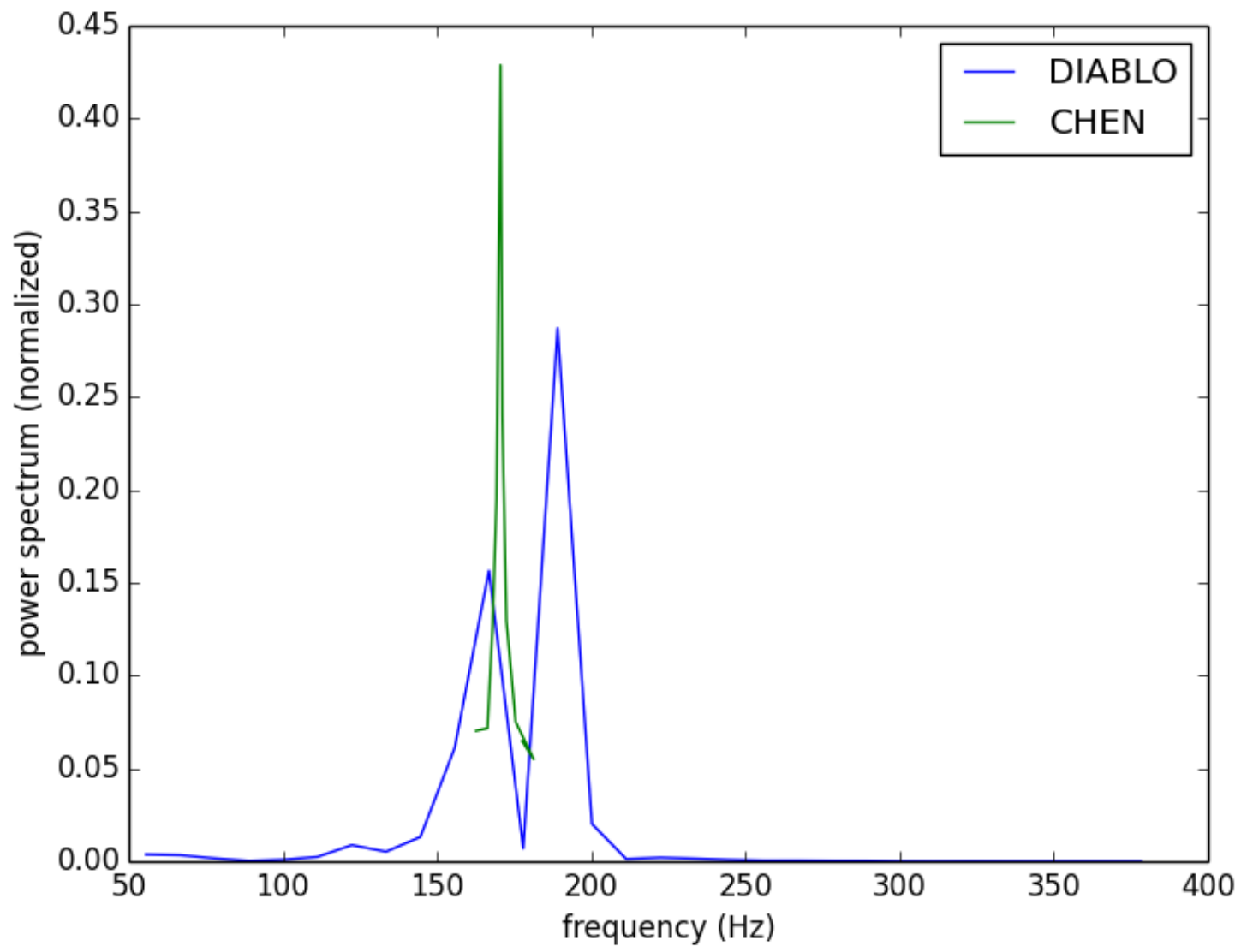

Figure 5.33. - Comparison of $1.28 \mathrm{~m} / \mathrm{s}$ out-of-plane frequency spectra, tube 15A-1, 1.5\% damping. 


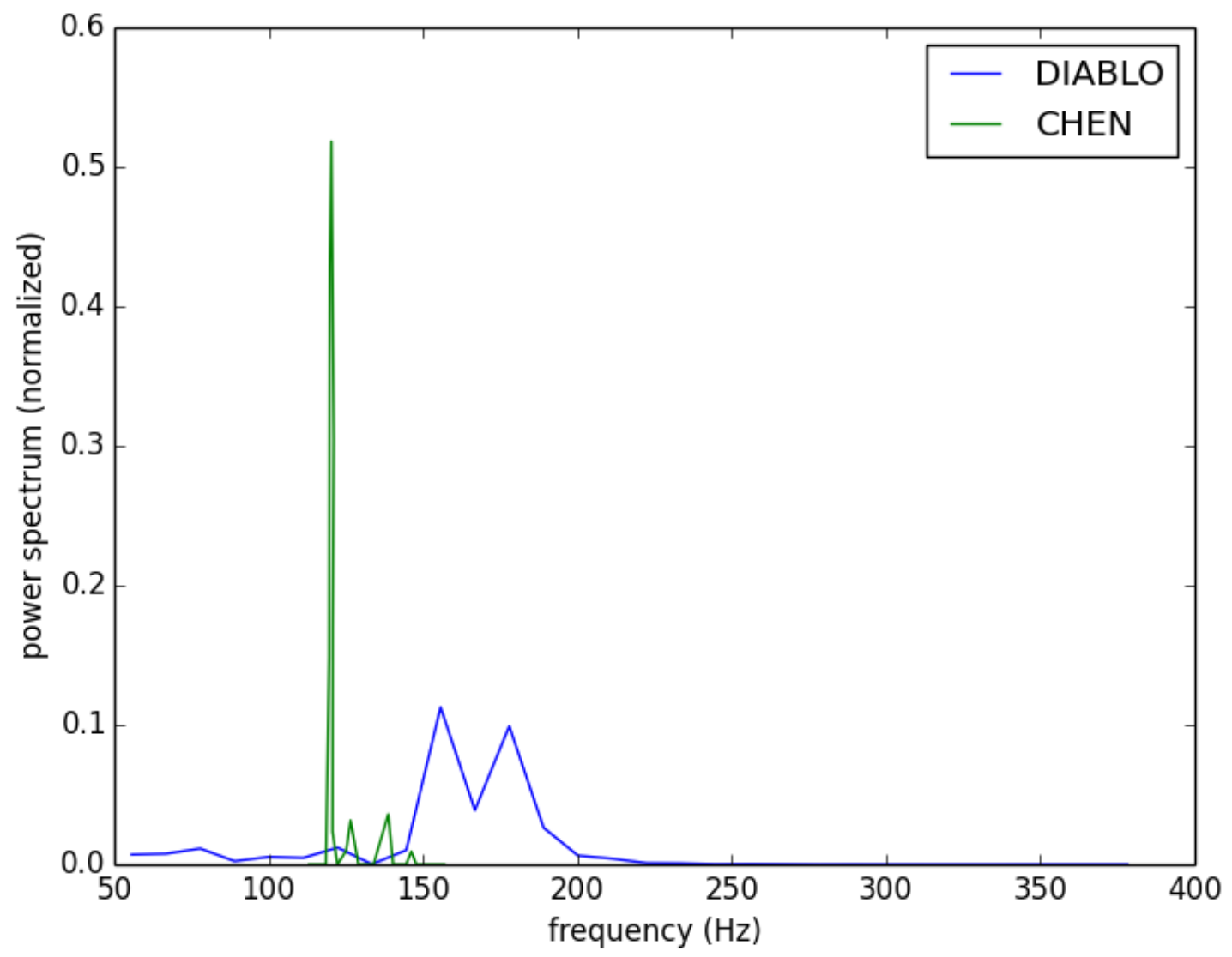

Fig. 5.34. - Comparison of $1.28 \mathrm{~m} / \mathrm{s}$ out-of-plane frequency spectra, tube 15B-1, $1.5 \%$ damping. 


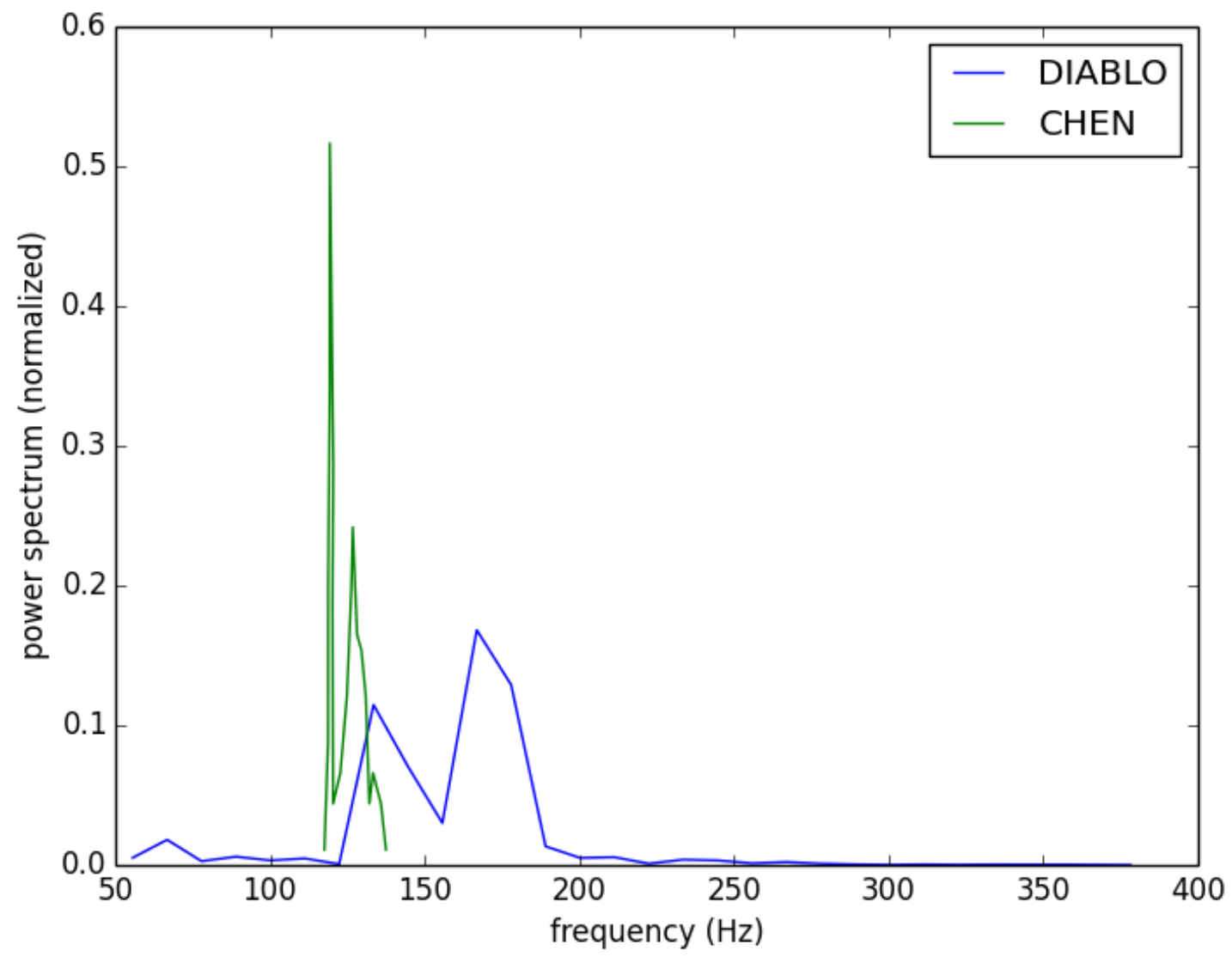

Fig. 5.35. - Comparison of $1.28 \mathrm{~m} / \mathrm{s}$ out-of-plane frequency spectra, tube 16-1, $1.5 \%$ damping.

\subsection{B\&W Dataset}

As well as the Argonne dataset, we performed three types of simulations for the B\&W dataset: fluid, structural, and coupled.

\subsubsection{Fluid Simulations}

We next describe work focused on the B\&W dataset [6] corresponding to a large helical tube bank in cross flow comprising 5 sections. Only three sections contained fluid flow. The three fluid sections have been simulated separately.

Several mesh iterations were attempted. An example of the mesh is provided in Fig. 5.36. ICEM generated meshes allowed for better resolution of boundary layers in the Cubit-generated mesh, the use of lower polynomial order and larger time steps. The boundary conditions are Inlet/Outlet (turbulent inflow -Synthetic Eddy Method, turbulent outflow) similar to that discussed in 5.1.1. The inlet velocity of interest is $0.6096 \mathrm{~m} / \mathrm{s}(2 \mathrm{ft} / \mathrm{s})$. Each section was modeled with 200,000-300,000 elements. The chosen polynomial order is 8 , showing no significant change when compared with the 6 polynomial order calculations. Each section was therefore modeled with 150,000,000 grid points. 
Pressure and velocity magnitude plots are shown in Fig. 5.37 and Fig. 5.38 for one of the sections.

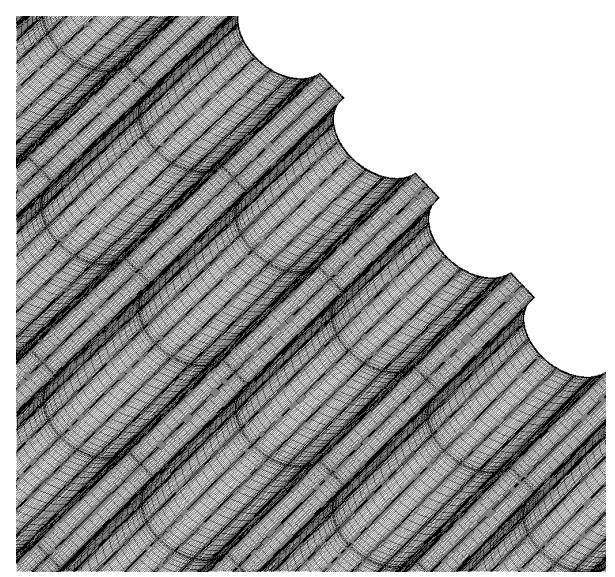

Fig. 5.36. Nek5000 example mesh with details of the surface mesh.
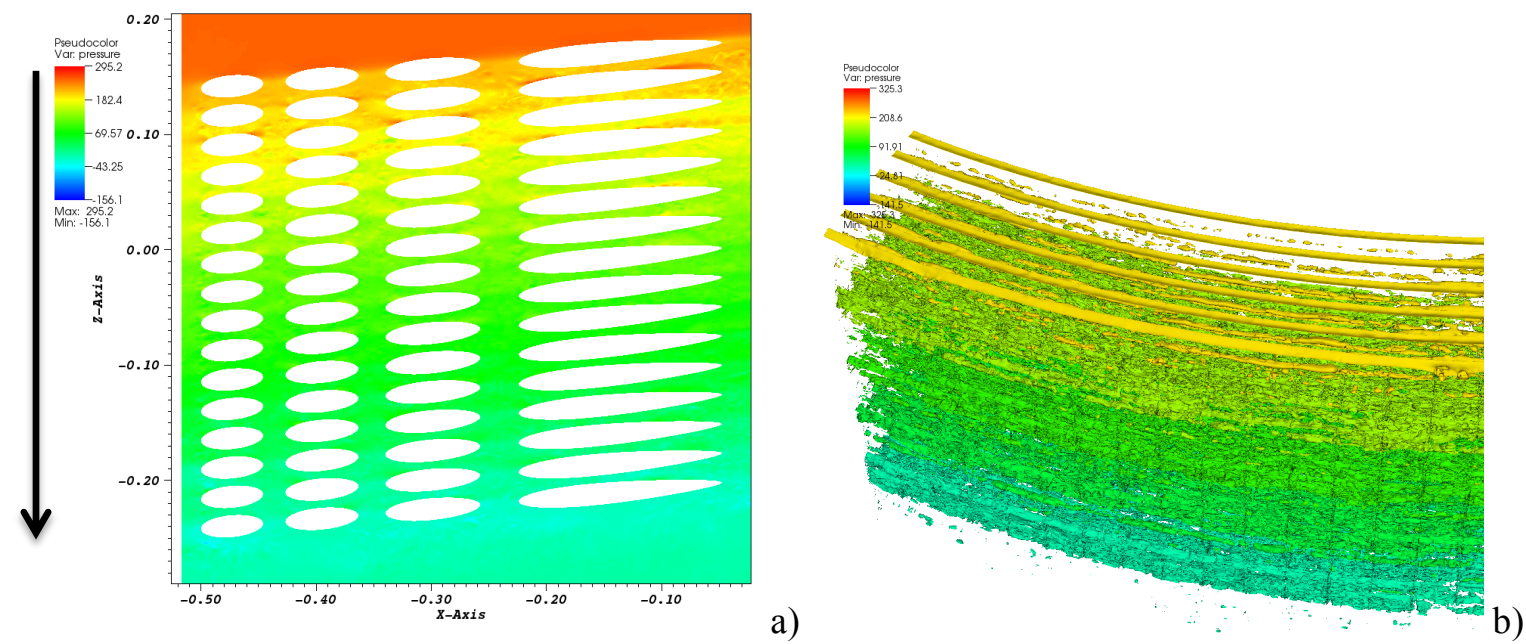

Fig. 5.37. Nek5000 calculations. Pressure distribution. a) cross section in the direction parallel to the streamwise direction, b) iso-surface plot. The arrows represent the flow direction. 

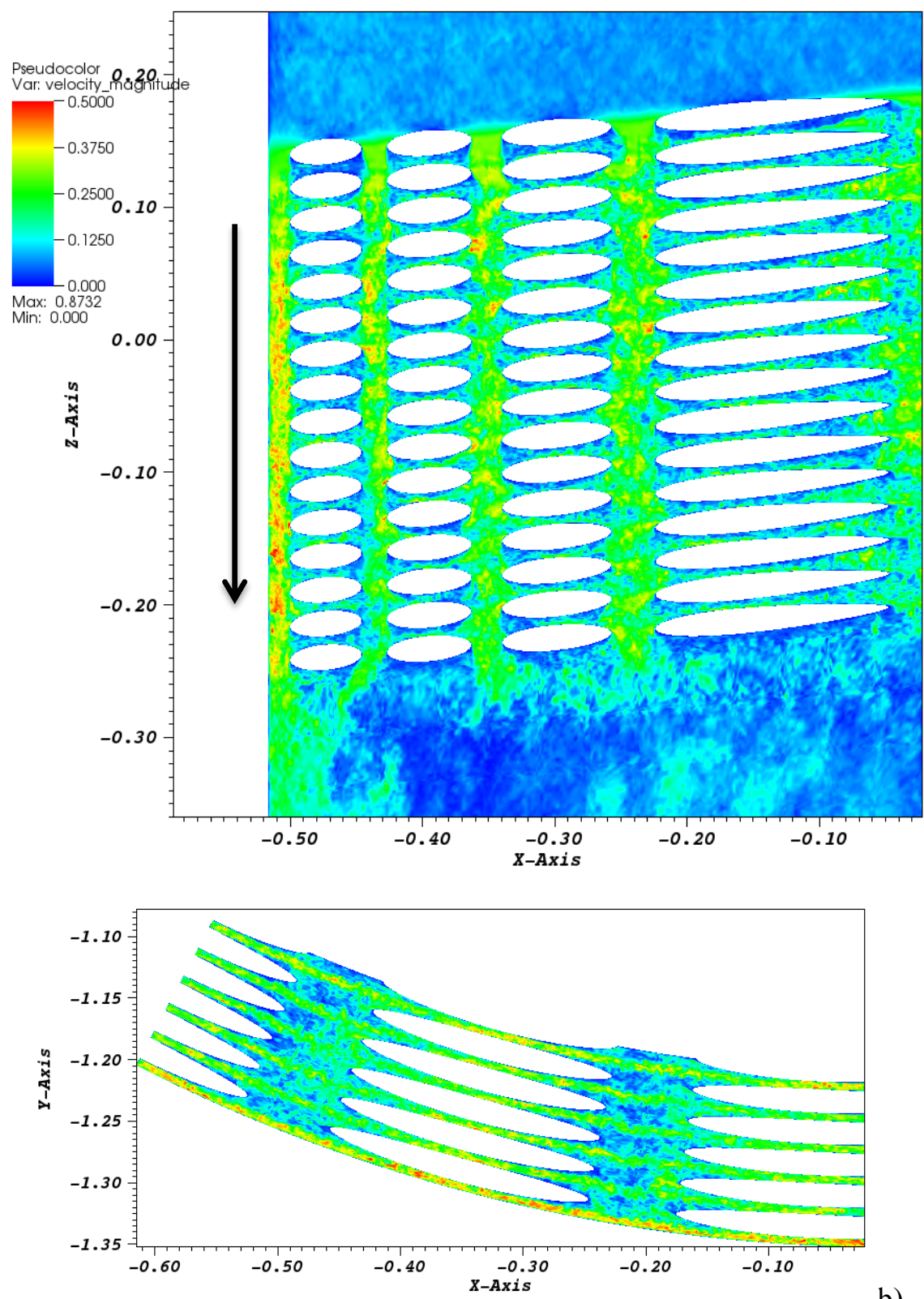

a)

b)

Fig. 5.38. Nek5000 calculations. Velocity magnitude. a) cross section parallel to streamwise direction, b) cross section normal to the streamwise direction. The arrows represent the flow direction.

\subsubsection{Structural Simulations}

Significant insight into the structural response is available via modal analysis. The B\&W test section itself consisted of 80 tubes arranged in a rectilinear grid, corresponding to tube ranks 5 ranks of tubes, with each rank consisting of 16 rows of tubes stacked vertically. In both the test and the fluid solver, an inner tube rank of $1 / 2$ tubes was was welded to the inner wall of the test chamber. 
The test chamber spanned 150 degrees, and was separated into 5 sections, each corresponding to an arcsection of the full steam generator, with arc sections varying between 28 and 32 degrees. In between each of the sections are 4 support structures, with support structure 1 separating section 1 and 2, support structure 2 separating sections 2 and 3, support structure 3 separating sections 3 and 4, and support structure 4 separating sections 4 and 5. At either end of the tubes (e.g. the ends of sections 1 and 5) the tubes were fixed to the sides of the test chamber.

In the test, the support structures were weldments manufactured from structural steel, one per tube rank. The weldments were affixed to the inner wall of the test chamber. The mesh for the structural approximation modeled the tubes as shell structures with a constant wall thickness. The support structures themselves were modeled by using 8-node hexahedra as solid blocks with cylindrical perforations corresponding to each of the tubes, and half-cylindrical perforations corresponding to where the inner rank "half-tube" would go. As in the actual test, the structural supports were fixed to the inner shell diameter. The tubes themselves are continuous (including through the perforations in the supports) for the entire 150-degree span. At each end of the span the tubes are fixed to the ends of the test chamber. Within the supports themselves, the tube displacements are tied to the nodal displacements of the solid element nodes that make up the surface of the tube perforations.

Because the arc-angle is small, to first order one may analyze the tubes as a simple straight Euler beam. The tube supports themselves cannot be represented either as a fully-clamped boundary condition (where the tubes in each span are isolated from one another), or a pinned condition (where the beam rotations and moments are continuous adjacent sections). Let $\mathrm{D}_{\mathrm{o}}$ be the outer tube diameter and $\mathrm{D}_{\mathrm{i}}$ the inner tube diameter. Then, the area moment of inertia of a simple tube satisfies

$$
I=\frac{\pi}{64}\left[D_{o}^{4}-D_{i}^{4}\right]
$$

Let $\mathrm{E}$ denote Young's modulus and $\rho_{1}$ the density per unit length. The smallest natural frequency should be associated with the longest tube length, in this case corresponding to the greatest arc-section. Letting $\mathrm{L}$ denote the length of a straight tube with the same arc-length as the section and assuming a fixed-fixed connection. Then the principal natural frequency of the vibration of such a tube can be calculated by using the following formula:

$$
f=\frac{1}{2 \pi}\left[\frac{22.37}{L^{2}}\right] \sqrt{\frac{E I}{\rho_{l}}}
$$

With this formula the principal natural frequencies are compiled in the table 5.5 below.

Table 5.5.. Analytical natural frequencies for straight tubes with lengths corresponding to arcsections

\begin{tabular}{|l|l|l|}
\hline Arc-section & $\begin{array}{l}\text { Fixed-fixed } \\
\text { frequency }(\mathrm{Hz})\end{array}$ & $\begin{array}{l}\text { Pinned-pinned } \\
\text { frequency }(\mathrm{Hz})\end{array}$ \\
\hline 28 degrees & 245.2 & 108.2 \\
\hline 30 degrees & 213.6 & 94.2 \\
\hline 32 degrees & 187.7 & 82.8 \\
\hline
\end{tabular}


However, the interior support structures themselves have a finite width. Hence the effective span length for sections 2, 3, and 4 is approximately one support structure width less than the nominal value, and the effective span length for sections 1 and 2 is approximately half support structure width less than the nominal value. Using these span length reductions, one can calculate the principal natural frequencies for each of the span lengths, as reported in Table 5.6 below.

Table 5.6. Analytical natural frequencies for straight tubes corresponding to the sections

\begin{tabular}{|l|l|l|l|l|}
\hline Section & Arc-section & $\begin{array}{l}\text { \# interior } \\
\text { supports }\end{array}$ & $\begin{array}{l}\text { Fixed-fixed } \\
\text { frequency }(\mathrm{Hz})\end{array}$ & $\begin{array}{l}\text { Pinned-pinned } \\
\text { frequency }(\mathrm{Hz})\end{array}$ \\
\hline 1 & 28 degrees & 1 & 262.7 & 115.9 \\
\hline 2 & 32 degrees & 2 & 212.2 & 93.6 \\
\hline 3 & 28 degrees & 2 & 282.3 & 124.5 \\
\hline 4 & 30 degrees & 2 & 243.5 & 107.5 \\
\hline 5 & 32 degrees & 1 & 199.4 & 88.0 \\
\hline
\end{tabular}

An eigenvalue calculation for the full structure is a messy proposition: since so many tubes are similar to each other, many modes have almost exactly the same natural frequency. Hence, in order to get a clear picture of the structural response, the structural mesh was modified to delete all the tubes except for that corresponding to the tube closest to the bottom outward corner. An eigenvalue analysis was then performed, and the first 10 eigenvalues were extracted. Of these, the most interesting are the first 5 eigenvalues, which are reported in Table 5.7.

Table 5.7. Eigenvalue as function of mode number.

\begin{tabular}{|c|l|l|l|}
\hline Mode & Angular & Temporal frequency & Period frequency \\
\hline 1 & $1.13458171 \mathrm{E}+03$ & $1.80574287 \mathrm{E}+02$ & $5.53788702 \mathrm{E}-03$ \\
\hline 2 & $1.18014698 \mathrm{E}+03$ & $1.87826226 \mathrm{E}+02$ & $5.32407014 \mathrm{E}-03$ \\
\hline 3 & $1.35076084 \mathrm{E}+03$ & $2.14980264 \mathrm{E}+02$ & $4.65158978 \mathrm{E}-03$ \\
\hline 4 & $1.49366505 \mathrm{E}+03$ & $2.37724176 \mathrm{E}+02$ & $4.20655576 \mathrm{E}-03$ \\
\hline 5 & $1.56092840 \mathrm{E}+03$ & $2.48429471 \mathrm{E}+02$ & $4.02528732 \mathrm{E}-03$ \\
\hline
\end{tabular}

These modes correspond to the principal modes of the five arc-sections. Pictures of the first five modes are included as figures 5.39-5.43. 


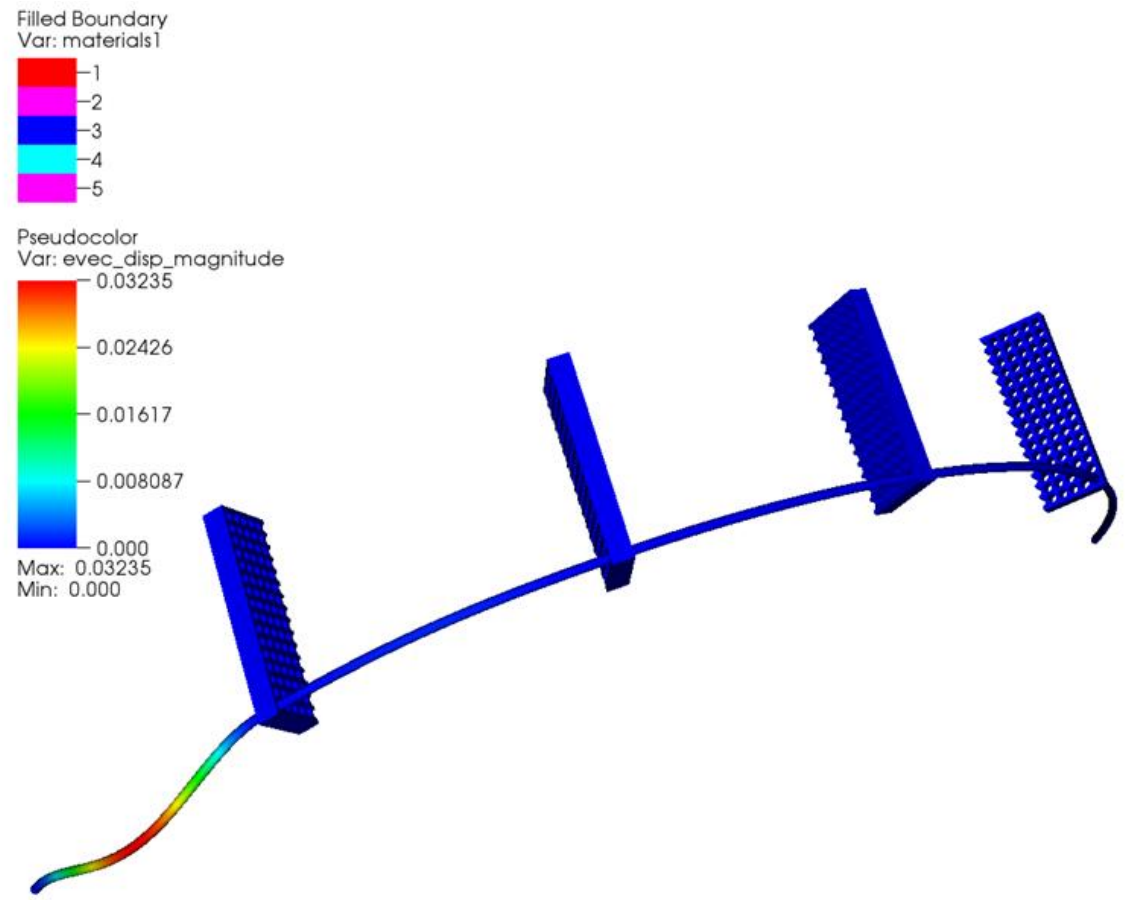

Fig. 5.39. First mode, frequency $180.574 \mathrm{~Hz}$, primary mode of section 5 .

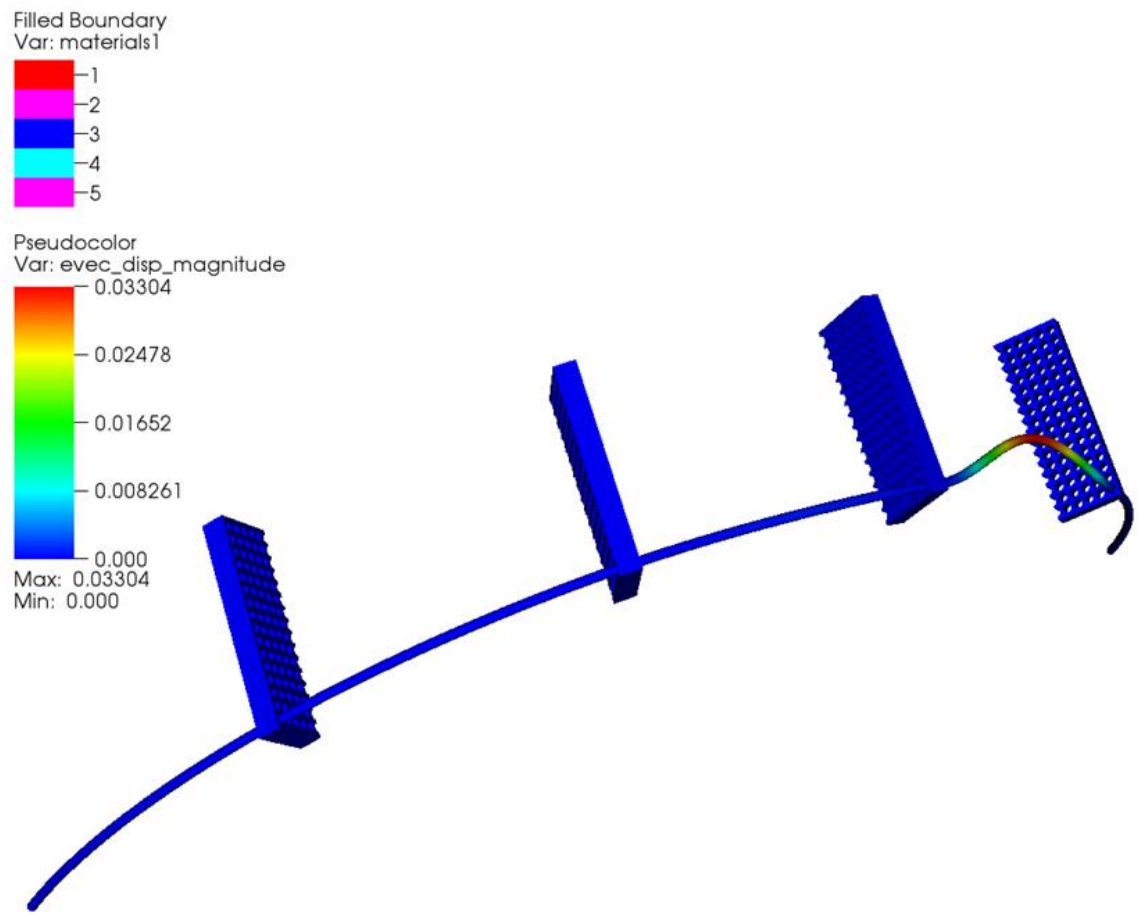

Fig. 5.40. Second mode, frequency 187.826, primary mode of section 2. 


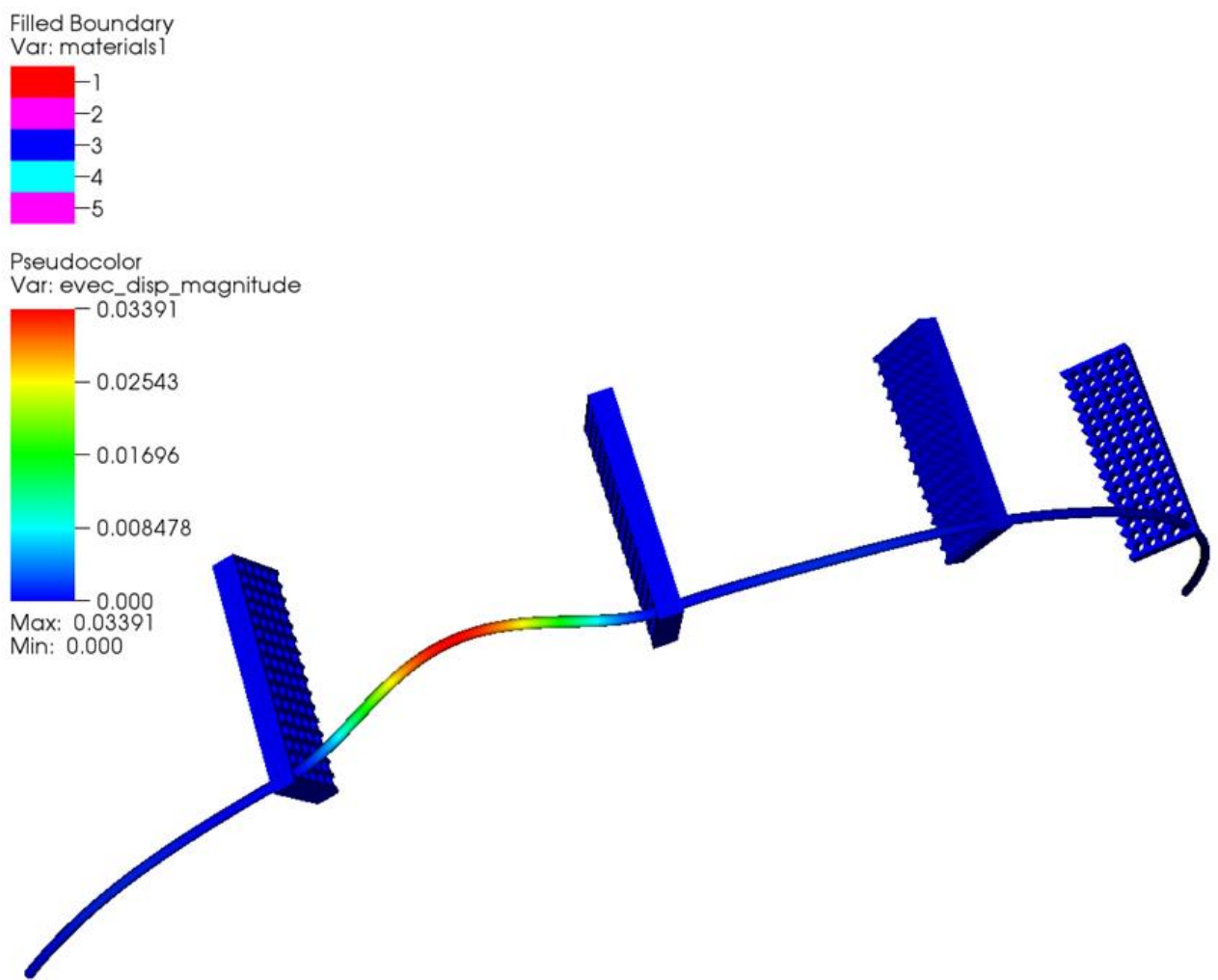

Fig. 5.41. Third mode, frequency $214.98 \mathrm{~Hz}$, primary mode of section 3 .

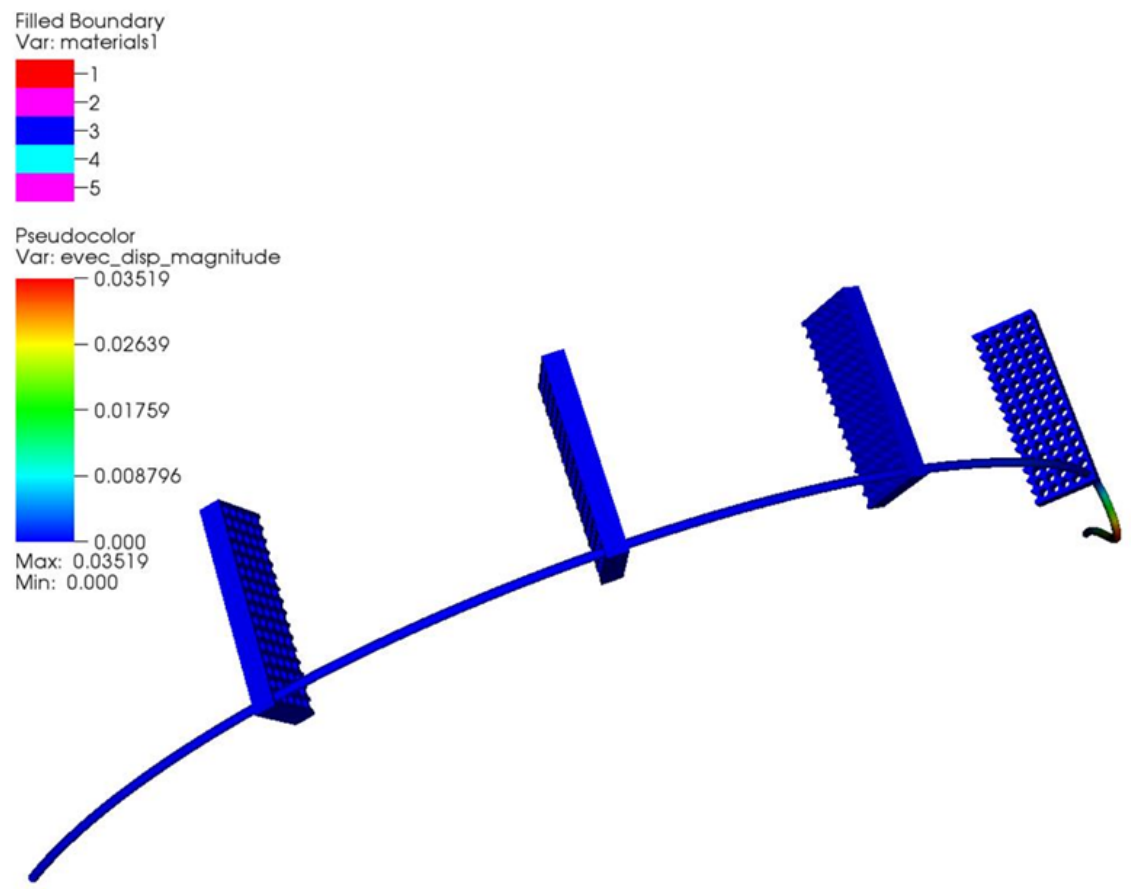

Fig. 5.42. Fourth mode, frequency $237.714 \mathrm{~Hz}$, primary mode of section 1 . 


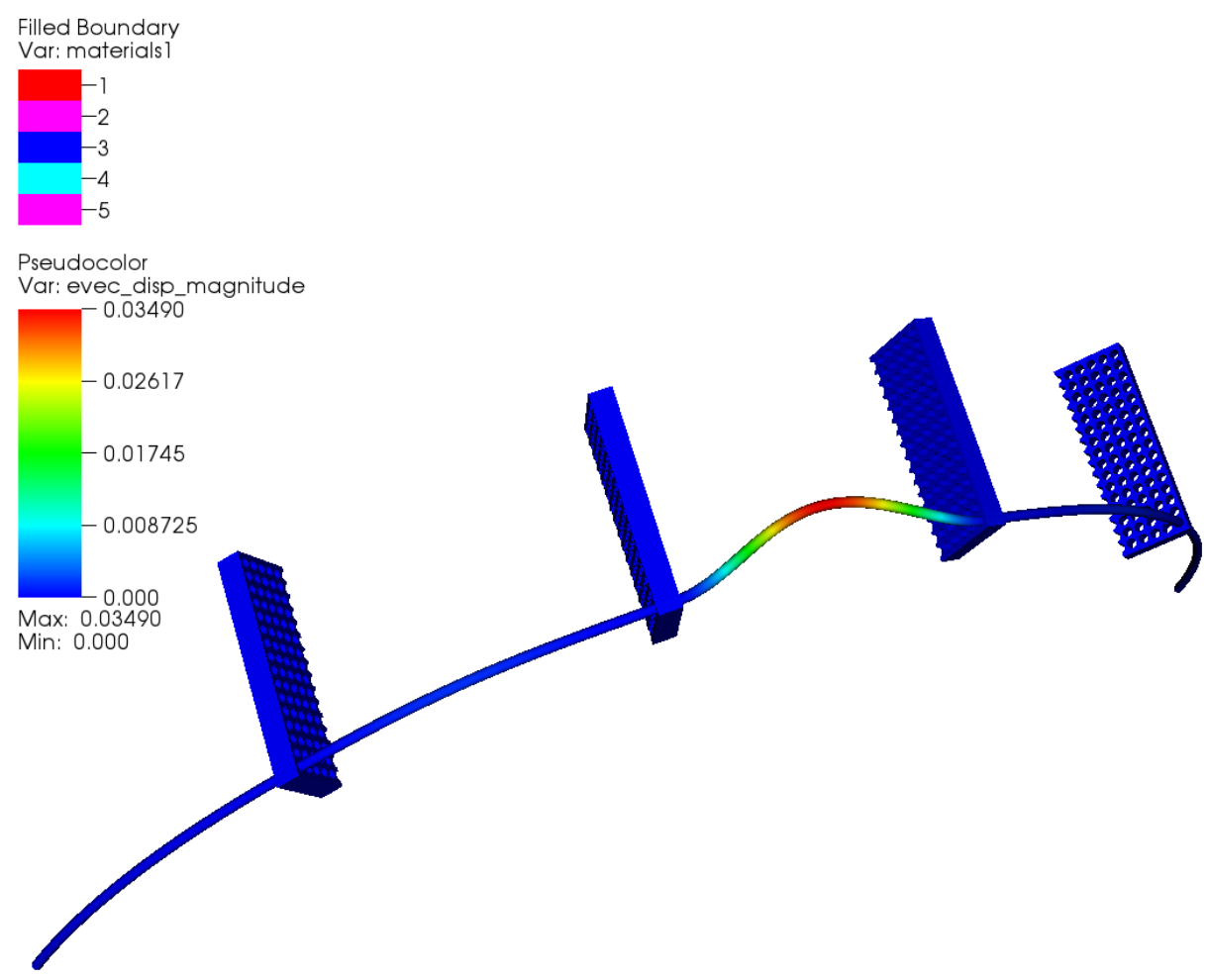

Fig. 5.43. 5th mode, frequency $248.429 \mathrm{~Hz}$, primary mode of section 3 .

The finite element results generally agree with analytical expectations regarding both the magnitude of the natural frequencies and the mode shapes. In general, however, the finite element model exhibits lower natural frequencies than the analytical results, noting once more that the analytical results are for straight tubes with the same lengths. There are three reasons for this: the support structures themselves are not infinitely stiff, the tubes themselves are curved, and the tubes are arranged with a slight pitch that leads to a length slightly longer than the nominal value. All these conditions either alone or in combination should lead to a lower natural frequency. Experimentally, the principal lowest natural frequencies were found to be somewhat lower, indicating that matching the experiment requires more detailed representation of the support structures and the support structure/tube interfaces. We also note that there exist analytical solutions for curved arches that allow for the inclusion of various end-conditions (including end conditions with finite stiffness). These should also be investigated for comparison with the finite element results.

\subsubsection{Coupled Calculations}

We performed one-way coupled analysis using the full system model, with flow through the center three sections. In lieu of any additional information, we used an added mass ratio of 1.47 as in the Argonne case (the tube sizes are relatively comparable).

We performed two different analyses using Rayleigh Damping, chosen to be $0.75 \%$ and $1.5 \%$ (as in the Argonne case), centered between $100 \mathrm{~Hz}$ and $200 \mathrm{~Hz}$. The approach flow velocity was set to $0.6096 \mathrm{~m} / \mathrm{s}$ $(2 \mathrm{ft} / \mathrm{s})$. The full system model contained 1,564,874 nodes and 1,567,472 elements. We initialized the 
problem statically using a time-averaged value of the pressure, the dynamic analysis then began from the static displacement.

The supports utilized using 8-node hexahedral elements employing "B-bar" reduced integration. The tubes employed using 4-node Hughes-Liu shell elements. Over 1200 Nek snapshots (at an interval of $3.0 \mathrm{E}-4 \mathrm{~s}$ ) mapped from Nek-5000 at time steps of 1.0e-4 were loaded into Diablo. Diablo itself utilized time steps of 5.0e-4, and implicit Newmark time integration with factors beta $=0.25$ and gamma $=0.5$. The Diablo calculations were performed on the Lawrence Livermore National Laboratory machine "cab", on 32 nodes, 1 processor-per-node, using the direct solver library PWSMP. Though the analysis can continue, the initial analyses progressed for 720 time steps up to $0.36 \mathrm{~s}$ of simulation time.

We chose two points ("A" and "B") to investigate for time history data, corresponding to location 30-1, $2 \mathrm{~b}$ and location 26-1, 2b. We investigated the out-of-plane displacement (in the paper called the "D", or Drag direction). The locations are illustrated in Fig. 5.44, where the initial displacement from the static initialization is also illustrated. Note the rather large initial displacement of tube 30-1 (location "B"). This may indicate that a longer time is needed for averaging in order to reduce initial flow perturbations. We compare our RMS displacement data to the experimental results Table 5.8. We are getting the magnitudes correct. Unfortunately, limited data exists from the data as Appendix A has not yet been located.

Table 5.8. RMS Tube Accelerations compared to BW experimental results.

\begin{tabular}{|c|c|c|c|}
\hline Tube & $\begin{array}{c}\text { Diablo RMS tube "D" (out-of- } \\
\text { plane) displacement (mil) } \\
\text { at 2 fps, } 0.075 \% \text { damping }\end{array}$ & $\begin{array}{c}\text { Diablo RMS tube "D" (out-of- } \\
\text { plane) displacement (mil) } \\
2 \mathrm{fps}, 1.5 \% \text { damping }\end{array}$ & $\begin{array}{c}\text { Experimental RMS tube } \\
\text { displacement (mil) } \\
2 \text { fps, from }\end{array}$ \\
\hline $26-1$ & 0.0206 & 0.0192 & N/A \\
\hline $30-1$ & .0551 & .0478 & 0.05 \\
\hline
\end{tabular}




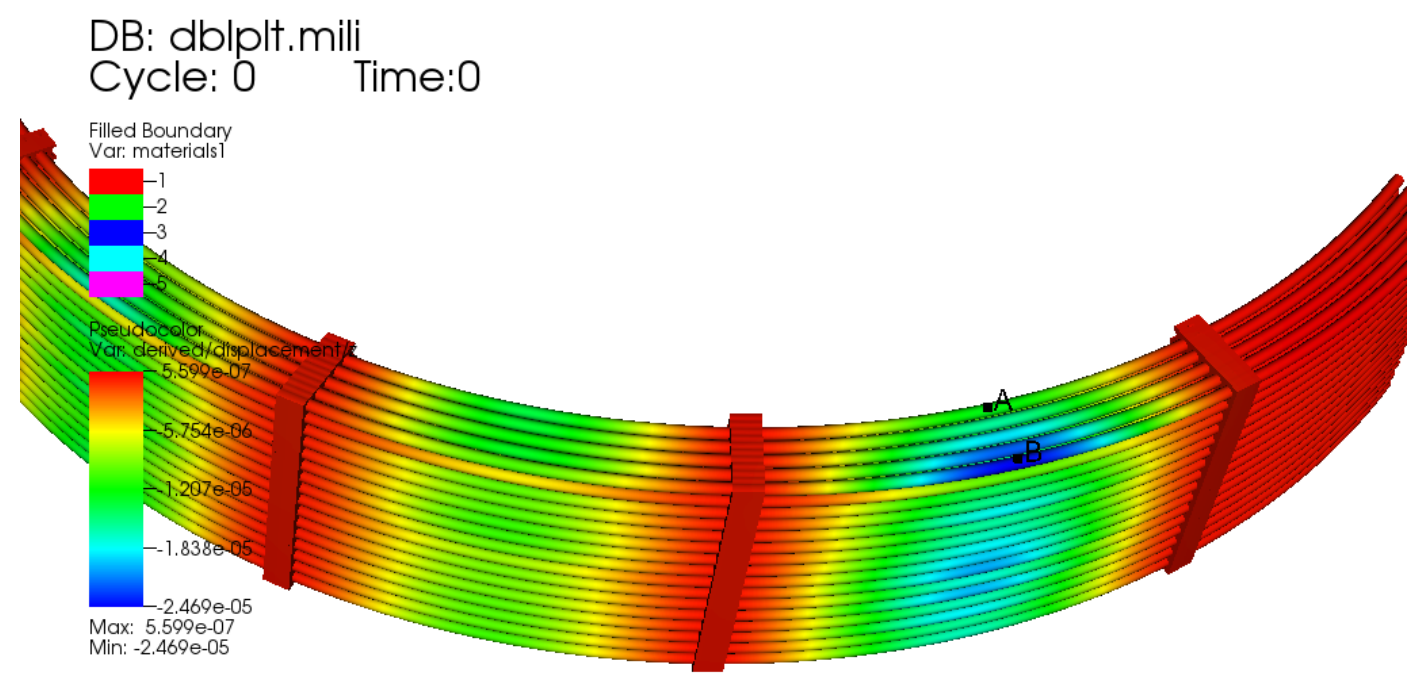

Fig. 5.44. - Positions of Diablo output for the BW SG, $A=$ Tube 26-1, B=Tube 30-1. The colors indicate the static displacement calculated by a time-averaged value of the pressure.

\section{Conclusions}

In this report we discussed the application of SHARP to legacy datasets, in preparation for its application to the NuScale design. This work represents an important stepping stone toward the ultimate goal of simulating helical steam generators at high fidelity.

Simulations were performed in the turbulent buffeting regime for two datasets: the B\&W dataset [6] and for the Argonne dataset [7]. The modeling approach relies on one-way coupling, which is considerably cheaper because it does not require sub-iterations. For both datasets, at low-flow conditions (i.e., such as those expected in SMRs) reasonable damping choices in the structural model are able to bound the experiment with the calculation results. Moreover, the spectral response is in reasonable agreement with the experiment.

For the Argonne dataset, a higher flow condition is also simulated, with, as expected, less success. For higher flow rates, approaching fluid elastic instability, it is expected that a tightly coupled capability is needed. Overall, the simulations performed show that a one-way methodology may be suitable for characterizing the fluid-structure response of helical steam generators at low flow conditions.

Further work will be dedicated to simulating the Argonne legacy dataset at higher flow conditions in order to characterize the approach to fluid-elastic instability. This work will focus on tight coupling.

Further validation will also be performed purely on the fluid solution. We emphasize that the flow in helical steam generators, based on the present work and the concurrent TAMU experiment, is considerably more complex phenomenologically than previously envisioned. The work presented here, while fairly complete, will be greatly enhanced by the additional validation work being performed. Moreover we emphasize the effort ongoing toward fully coupled simulations ("two-way") currently ongoing under SHARP [28]. 


\section{References}

1. A. Siegel, T. Tautges, A. Caceres, D. Kaushik, P. Fischer, G. Palmiotti, M.A. Smith, J. Ragusa, "Software Design of SHARP," in Proceedings of the Joint International Topical Meeting on Mathematics and Computations and Supercomputing in Nuclear Applications $(M \& C+S N A)$, American Nuclear Society, April 2007.

2. Vijay S. Mahadevan, Elia Merzari, Timothy Tautges, Rajeev Jain, Aleksandr Obabko, Michael Smith, Paul Fischer, "High-resolution coupled physics solvers for analysing fine-scale nuclear reactor design problems", Philosophical Transactions of the Royal Society of London A, DOI:10.1098/rsta.2013.0381

3. Burman, E., Fernández, M., "Stabilization of explicit coupling in fluid-structure interaction involving fluid incompressibility," Comput. Methods Appl. Mech. Eng. (2008)

4. Bobovnik, G., Mole, N., Kutin, J., Stok, B., Bajsic, I., "Coupled finite volume/finite-element modelling of the straight-tube Coriolis flowmeter," J. Fluids and Structures, 785-800 (2005)

5. Stefan Turek, Jaroslav Hron "Proposal for Numerical Benchmarking of Fluid-Structure Interaction between an Elastic Object and Laminar Incompressible Flow", Fluid-Structure Interaction, Volume 53 of the series Lecture Notes in Computational Science and Engineering pp 371-385

6. Watson, G. B. "Functional performance of the helical coil steam generator, Consolidated Nuclear Steam Generator (CNSG) IV system - Final report", Report PB-253324; ARC-5178-F (1975)

7. S.S. Chen, J.A. Jendrzejczyk, M.W. Wambsganss, "Tube vibration in a half-scale sector model of a helical tube steam generator", Journal of Sound and Vibration, Volume 91, Issue 4, 22 December 1983, Pages 539-569

8. Mueller, A., "The validation of STAR-CCM+ coupled to Abaqus for analyzing fluid-elastic instabilities in a flexible tube bundle," STAR Global Conf. (2013)

9. T.J. Tautges, R. Meyers, K. Merkley, C. Stimpson, C. Ernst, MOAB: A Mesh-Oriented Database, Sandia National Laboratories report SAND2004-1592, April 2004.

10. T.J. Tautges, H.-J. Kim, A. Caceres, R. Jain, "Coupled Multi-Physics simulation frameworks for reactor simulation: A Bottom-Up approach," in Proceedings of the International Conference on Mathematics and Computational Methods Applied to Nuclear Science and Engineering (M\&C), American Nuclear Society, Rio de Janeiro, Brazil, May 2011.

11. D. Gaston, C. Newman, G. Hansen, D. Lebrun-Grandi, "MOOSE: a parallel computational framework for coupled systems of nonlinear equations," Nuclear Engineering and Design, 239(10):1768-1778, Oct. 2009

12. E Merzari, WD Pointer, P Fischer, "Numerical simulation and proper orthogonal decomposition of the flow in a counter-flow T-junction", Journal of Fluids Engineering, 135, paper 091304 (2013).

13. Fischer, P., Lottes, J., Kerkemeier, S., Marin, O., Heisey, K., Obabko, A., Merzari, Unpublished information. Argonne National Laboratory.

14. Y. Maday, A.T. Patera, "Spectral element methods for the Navier-Stokes equations," in A.K. Noor and J.T. Oden, editors, State-of-the-Art Surveys in Computational Mechanics, pp. 71-143, ASME, New York, 1989.

15. A.G. Tomboulides, J.C.Y. Lee, and S.A. Orszag, "Numerical simulation of low Mach number reactive flows," Journal of Scientific Computing, 12:139-167, June 1997.

16. A.G. Tomboulides, M. Israeli, G.E. Karniadakis, "Efficient removal of boundary-divergence errors in time-splitting methods," Journal of Scientific Computing, 4:291-308, 1989.

17. D. Parsons, J.M. Solberg, R.M. Ferencz, M.A. Havstad, N.E. Hodge, and A.P. Wemhoff, Diablo User Manual, Lawrence Livermore National Laboratory report UCRL-SM-234927, Sept. 2007.

18. D. J. Segalman "A Four-Parameter Iwan Model for Lap-Type Joints," Journal of Applied Mechanics, vol. 72, pp. 752-760, September 2005.

19. D. D. Quinn and D. J. Segalman, "Using Series-Series Iwan-Type Models for Understanding Joint Dynamics," Journal of Applied Mechanics, vol. 72, pp. 666-673, 2005.Daniel J. Segalman, 
Danny L. Gregory, Michael J. Starr,Brian R. Resor, Michael D. Jew, James P. Lauffer, \& Nicoli M. Ames , "Handbook on Dynamics of Jointed Structures", SANDIA REPORT SAND2009-4164, July 2009.

20. O.V. Shiryayev, S.M. Page, C.L. Pettit, J.C. Slater, "Parameter estimation and investigation of a bolted joint model", Journal of Sound and Vibration, Volume 307, Issues 3-5, 6 November 2007, Pages 680-697.

21. T.J. Royston, "Leveraging the equivalence of hysteresis models from different fields for analysis and numerical simulation of jointed structures", ASME J. Comput. Nonlinear Dynam., 3 (2008), pp. 031006-1-031006-8.

22. Ivan I. Argatov, Eric A. Butcher, On the Iwan models for lap-type bolted joints, International Journal of Non-Linear Mechanics, Volume 46, Issue 2, March 2011, Pages 347-356.

23. Yikun Li, Zhiming Hao, A six-parameter Iwan model and its application, Mechanical Systems and Signal Processing,Volumes 68-69, February 2016, Pages 354-365.

24. S. S. Chen, M. W. Wambsganss and J. A. Jendrzejczyk, "Added Mass and Damping of a Vibrating Rod in Confined Viscous Fluids", Journal of Applied Mechanics, Volume 43(2), 325-329 (Jun 01, 1976).

25. S. S. Chen and J. A. Jendrzejczyk, "Flow velocity dependence of damping in tube arrays subjected to liquid cross flow", Journal of Pressure Vessel Technology 103, pp. 130-135, 1981

26. M. Rahman, D.D. Bhatta, "Evaluation of added mass and damping coefficient of an oscillating circular cylinder", Applied Mathematical Modelling, Volume 17, Issue 2, 1993, Pages 70-79.

27. M. J. Pettigrew, R. J. Rogers and F. Axisa, "Damping of Heat Exchanger Tubes in Liquids: Review and Design Guidelines", Journal of Pressure Vessel Technology, Volume 133(1), 014002 (Jan 21, 2011).

28. Elia Merzari, Jerome Solberg, Paul Fischer, Robert M Ferencz, "A high-fidelity approach for the simulation of flow-induced vibration", Proceedings of the ASME 2016 Fluids Engineering Division Summer Meeting, pp. V01AT03A019-V01AT03A019, (2016) 


\section{Argonne}

Nuclear Engineering Division

Argonne National Laboratory

9700 South Cass Avenue, Bldg. 208

Argonne, IL 60439

www.anl.gov 